\title{
Knowledge of Content for Clinical Nursing Educators: An Ethnographic Investigation of Clinical Experts Who Transition to the Role of Novice Clinical Nursing Educator
}

\author{
Stacy Wheat Huber
}

swhuber@hsc.wvu.edu

Follow this and additional works at: https://researchrepository.wvu.edu/etd

Part of the Higher Education and Teaching Commons, and the Nursing Commons

\section{Recommended Citation}

Huber, Stacy Wheat, "Knowledge of Content for Clinical Nursing Educators: An Ethnographic Investigation of Clinical Experts Who Transition to the Role of Novice Clinical Nursing Educator" (2020). Graduate Theses, Dissertations, and Problem Reports. 7626.

https://researchrepository.wvu.edu/etd/7626

This Dissertation is protected by copyright and/or related rights. It has been brought to you by the The Research Repository @ WVU with permission from the rights-holder(s). You are free to use this Dissertation in any way that is permitted by the copyright and related rights legislation that applies to your use. For other uses you must obtain permission from the rights-holder(s) directly, unless additional rights are indicated by a Creative Commons license in the record and/ or on the work itself. This Dissertation has been accepted for inclusion in WVU Graduate Theses, Dissertations, and Problem Reports collection by an authorized administrator of The Research Repository @ WVU.

For more information, please contact researchrepository@mail.wvu.edu. 
Knowledge of Content for Clinical Nursing Educators: An Ethnographic Investigation of Clinical Experts Who Transition to the Role of Novice Clinical Nursing Educator

Stacy Wheat Huber

Dissertation submitted to the College of Education and Human Services at

\author{
West Virginia University \\ in partial fulfillment of the requirements for the degree of \\ Doctor of Education in Curriculum and Instruction.
}

\author{
Keri Valentine, Ph.D., Chair \\ Sharon Hayes, Ph.D \\ Melissa Luna, Ph.D \\ Kari Sand-Jecklin EdD \\ Department of Curriculum and Instruction
}

Morgantown, West Virginia

2020

Keywords: nursing education, clinical nursing educators, teaching practices Copyright 2020 Stacy Wheat Huber 


\begin{abstract}
Knowledge of Content for Clinical Nursing Educators: An Ethnographic Investigation of

Clinical Experts who Transition to the Role of Novice Clinical Nursing Educator.
\end{abstract}

Stacy Wheat Huber

The purpose of this research study was to identify and describe the teaching practices and the grounding experiences of the teaching practices novice educators use when teaching undergraduate students in the clinical patient care area. The following research questions guided this investigation: What teaching practices and teacher knowledge do novice clinical nursing educators demonstrate or draw from when teaching undergraduate nursing students in the clinical patient-care area? In what ways do novice clinical nursing educators change or adapt their teaching behaviors as a response to the context of the teaching/learning environment? What grounding educational or professional experiences do novice clinical nursing educators refer to or draw upon when working in the role of the clinical nursing educator? What are areas of teacher knowledge and/or teaching practices, utilized by novice clinical nursing educators, which preclude the facilitation of student learning and/or critical thinking? A focused ethnographic research methodology was used for this investigation. Data included approximately 70 hours of observation of the clinical teaching practices of four novice clinical nursing educators, as well as semi-structured interviews, and document collection. All data sources were analyzed though a theoretical thematic process that drew upon the Pedagogical and Subject Matter Knowledge for Clinical Nursing Educators framework, adapted from the Mathematical Knowledge for Teaching model. The analysis and interpretation of the data revealed that novice clinical nursing educators more often draw upon their subject matter knowledge rather than utilize their pedagogical knowledge when teaching in the clinical patient area. Findings also revealed that when teaching in the clinical patient care setting, novice clinical nursing educators may under-utilize teaching methods, such as questioning and reflection, to facilitate critical thinking. 


\section{Dedication}

I would like to dedicate this dissertation to Alex, Evan, and Jason. My whole world, my whole heart, and my whole life. 


\section{Acknowledgments}

I would like to acknowledge and thank my doctoral committee for their support and mentorship. I would like to express my sincerest thanks and gratitude to my dissertation chair Dr. Keri Valentine and my committee members Dr. Sharon Hayes, Dr. Melissa Luna, and Dr. Kari Sand-Jecklin. Thank you all for your time, detailed feedback, guidance, and especially for agreeing to work closely with me throughout this process. I would like to thank all of the talented educators at the WVU College of Education and Human Services for their guidance and for sharing their knowledge and experience with me throughout this process. I would like to especially thank the four wonderful and talented clinical nursing educators who were so kind to participate in this study. Finally, I would like to thank my nursing educator peers at the WVU SON for their support and encouragement over the past five years. Thank you all. 


\section{Table of Contents}

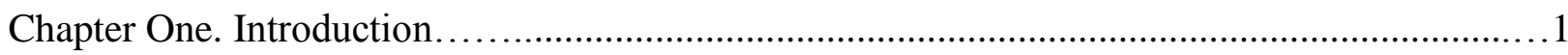

Introduction to the Problem........................................................

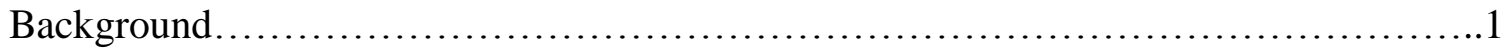

Nursing Faculty Shortage ...............................................

Nursing Shortage ..............................................................

Current Effort to Ease Nursing Shortage.......................................4

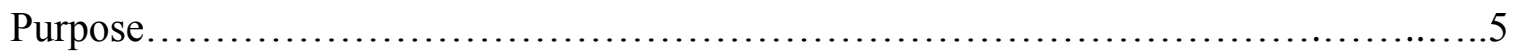

Motivation for the Investigation....................................................

Research Questions............................................................

Significance of the Investigation...............................................

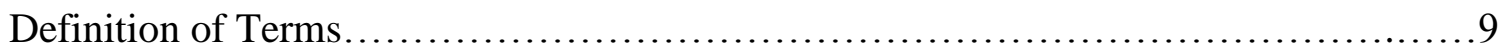

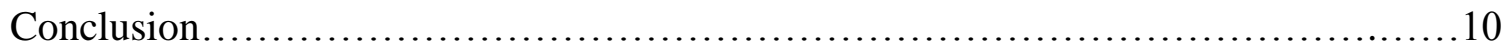

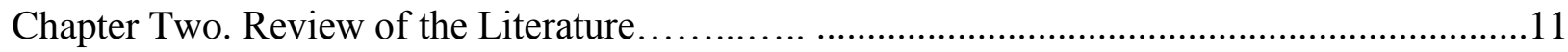

Introduction to the Literature Review............................................11

Preparation of a Nursing Educator................................................ 12

The Role of the Clinical Nursing Educator............................................14

Signature Pedagogies.................................................... 14

Facilitation of Learning ..................................................15

Perception of the Role of the Clinical Nursing Educator........................15

Knowledge of a Teacher...................................................... 16 
Subject Matter Knowledge................................................17

General Pedagogical Knowledge.........................................18

Contextual Knowledge.....................................................19

Pedagogical Content Knowledge for Clinical Teaching.......................20

Mathematical Knowledge for Teaching .....................................21

Subject Matter Knowledge........................................22

Pedagogical Content Knowledge...................................25

Clinical Teaching Practices.....................................................

Development of Critical Thinking ...................................... 30

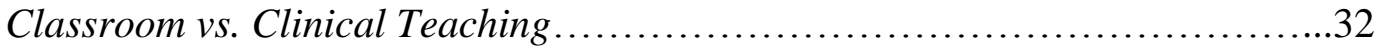

Clinical Pedagogies That Promote Critical Thinking ..........................32

Facilitate Pre-and-Post Conference Discussion........................33

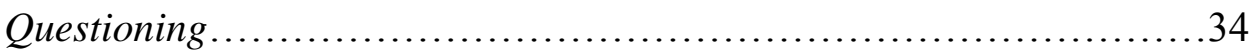

Role Modeling................................................ 36

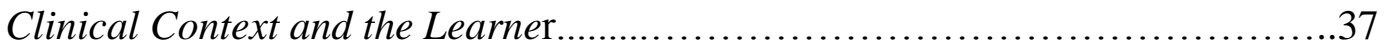

Clinical Context...................................................

Learning Opportunities............................................... 38

Developmental Level of the Learner................................39

Nursing Students Perspectives on Clinical Teaching .........................39

The Novice Clinical Nursing Educator.........................................41

Novice- to-Expert Model.............................................42

Situated Learning ..................................................44 
Legitimate Peripheral Participation.....................................45

Communities of Practice ..................................................46

Challenges to Learning to Teach on-the-Job ...................................47

Emotional Challenges................................................... 48

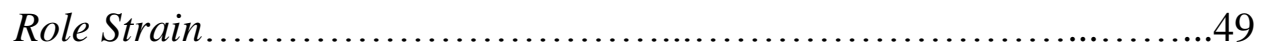

Role Ambiguity ........................................................49

Facing the Challenge of Learning-to-Teach-On-the-job.........................50

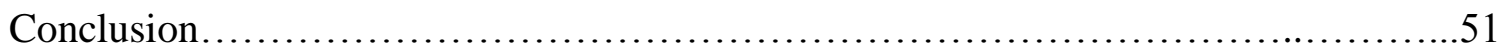

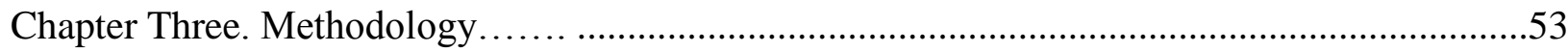

Introduction to the Research Methods .............................................53

Review of the Methodological Literature .............................................54

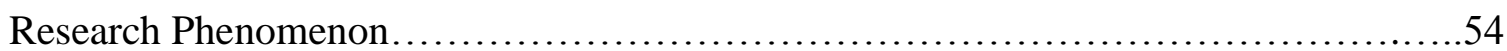

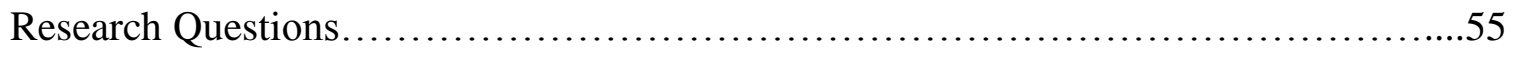

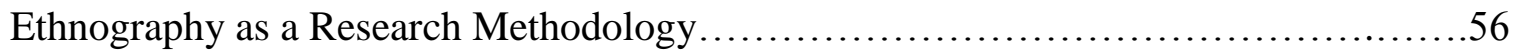

Focused Ethnography................................................ 58

Epistemological and Theoretical Perspective ..................................60

Research Study Design.........................................................

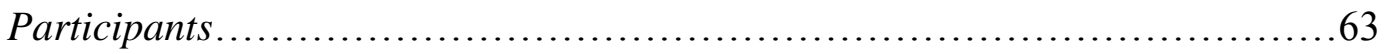

Number of Participants...................................................64

Introduction to the Participants .............................................64

Data Collection Methods.........................................................66

Field Work and Observational Setting...............................67

Time Frame.......................................................... 68 


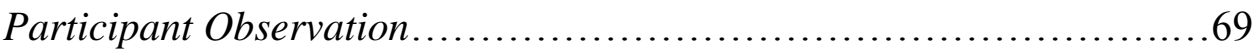

Student and Patient Confidentiality......................................71

Ethnographic Interviewing ....................................... 71

Semi-structured Interviewing ....................................72

Document Collection ................................................ 73

Reflective Journaling................................................74

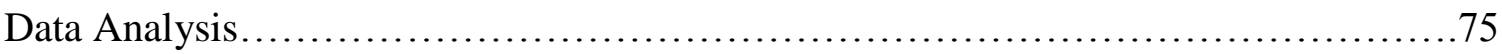

Theoretical Thematic Analysis............................................. 75

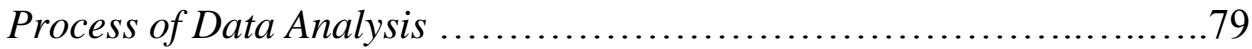

Generating Codes................................................... 80

Generalizing Themes ................................................. 81

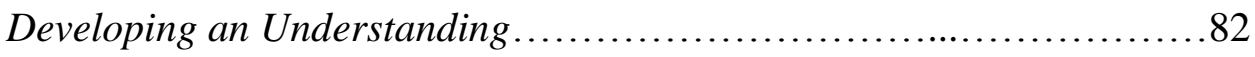

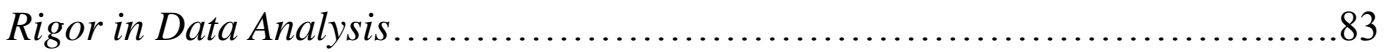

Credibility........................................................ 83

Dependability .................................................... 84

Confirmability ....................................................... 84

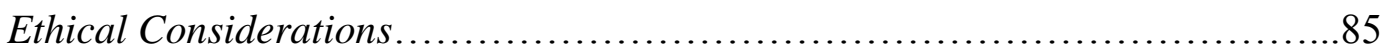

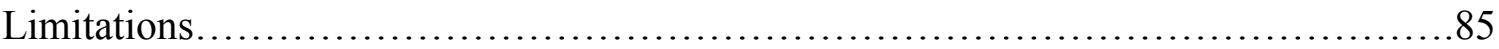

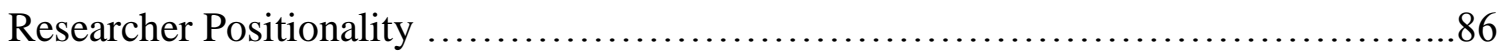

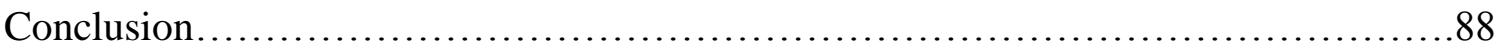

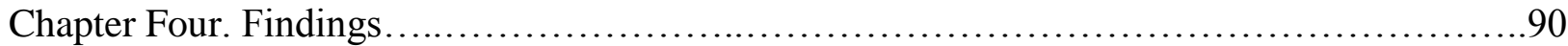

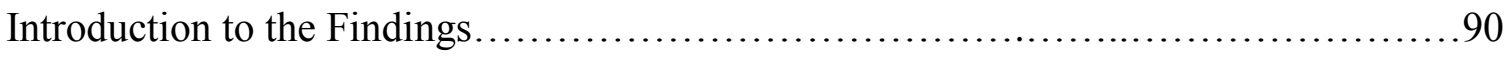

Section 1: Introduction to the Participants and Grounding Experiences..................91 
Alex's Grounding Experiences................................................92

Bailey's Grounding Experiences..........................................99

Chris's Grounding Experiences............................................97

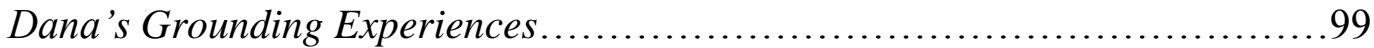

Summary of Grounding Experiences.........................................101

Summary of Section One ................................................... 102

Section Two: Teaching Practices Used in the Clinical Patient Care Setting..............103

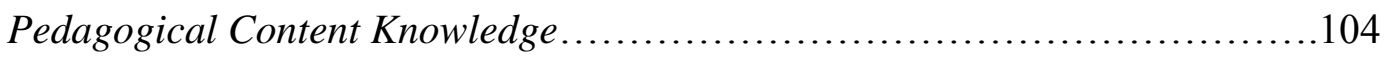

Knowledge of Content and Curriculum.................................104

Responding: Providing Directions for Completion of

Assignment..................................................105

Questioning: Asking Question Concerning Completion of

Assignment ................................................. 106

Responding: Setting Expectations for the Completion of Skills or

Tasks.................................................... 106

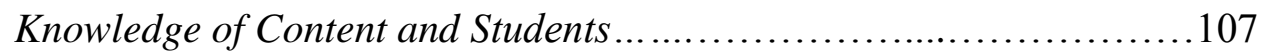

Responding: Using Unique Examples.............................108

Responding: Clarification of Misconceptions or

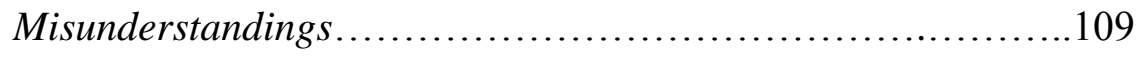

Knowledge of Content and Teaching...................................110

Responding: Teaching Through Personal Stories................111

Responding: Demonstration or Role

Modeling ....................................................112 
Responding: Creation of Original Learning Materials...........113

Responding: Classroom Management.......................114

Questioning: Engaging in Discussion and/or Reflection.........115

Questioning: Asking Knowledge or Recall questions.............117

Questioning: Asking Application or Critical Thinking

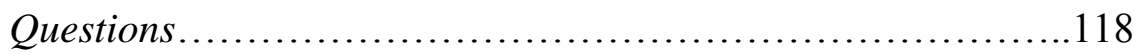

Summary of Pedagogical Content Knowledge.................................119

Subject Matter Knowledge..............................................120

Common Content Knowledge...................................121

Responding: Providing Clinical Rationale...................122

Responding: Using Clinical Judgment........................123

Responding: Providing Directions for Care...................124

Questioning: Asking for Additional Clinical Information..........124

Questioning: Asking about Completion of Tasks.................125

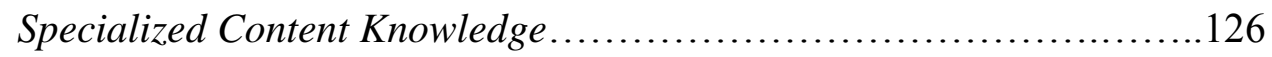

Responding: Providing Clinical Rationale....................127

Responding: Providing Directions for Care...................128

Responding: Using Clinical Judgment...................... 128

Questioning: Asking about Completion of Tasks.................129

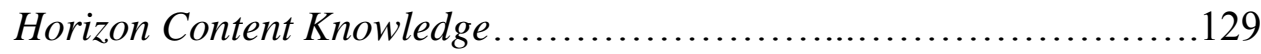

Summary of Subject Matter Knowledge....................................131

Summary of Section Two.............................................. 132

Section Three: Teaching Practices that Deterred Student Learning..................133 
Asking Questions That are Above the Level of the Learner......................133

Under-Utilization of Questioning as a Teaching Practice.......................135

Under-Utilization of Discussion or Reflection as a Teaching Practice............137

Summary of Section Three .............................................139

Similarities and Variances in Teaching Practices................................139

Summary of Major Findings................................................ 141

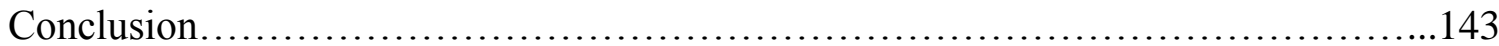

Chapter Five. Discussion and Conclusion........................................... 144

Introduction............................................................... 144

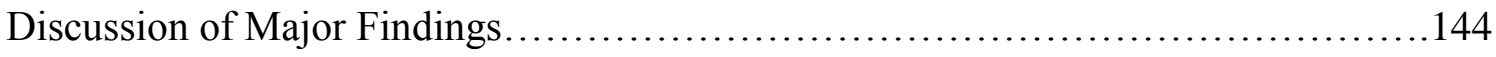

Influences on and the Perceptions of the Role of the Novice Clinical Nursing

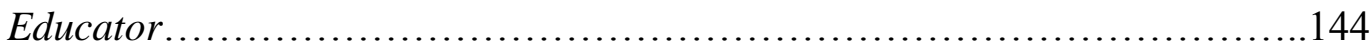

Under-utilization of Teaching Practices................................146

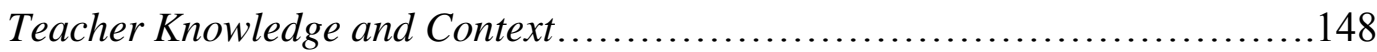

Limitations and Transferability........................................ 151

Conclusions and Recommendations for Future Practice..........................152

Conclusions and Recommendations for Future Research..........................153

Imagining the Possibilities..................................................... 155

Development of Horizon Content Knowledge................................156

Role of the Clinical Nursing Educator...................................157

Utilization of Simulation..............................................158

Final Thoughts......................................................... 159

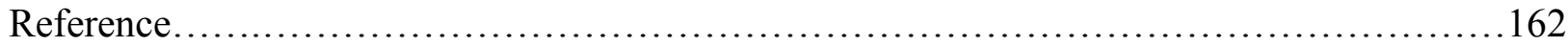


Appendixes................................................................. 182

Appendix A: Themes and Codes..........................................182

Appendix B: Themes and Framework..................................... 184

\section{List of Tables}

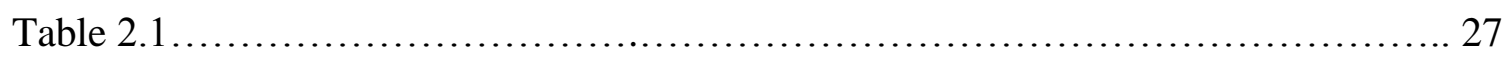

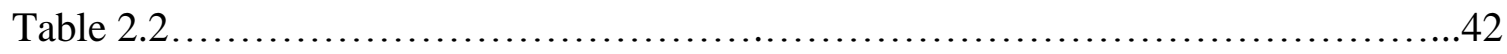

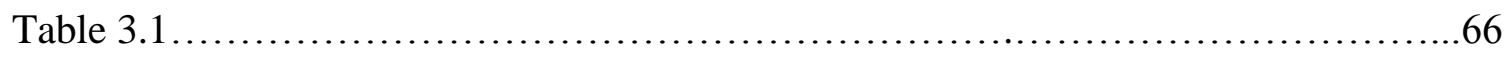

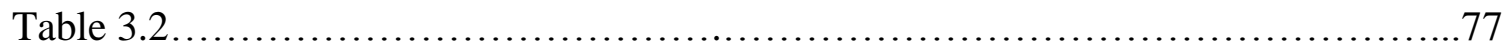

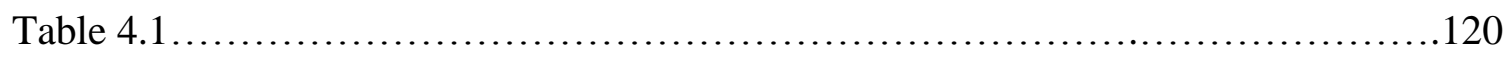

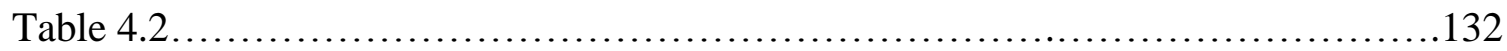

\section{List of Figures}

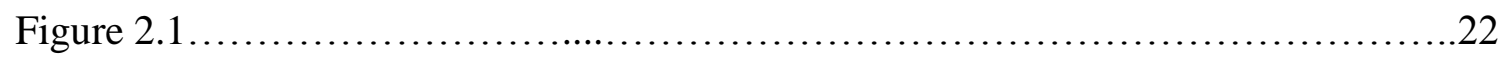




\section{Chapter One \\ Introduction}

\section{Introduction to the Problem}

Due to the demand for qualified registered nurses needed to meet the healthcare needs of the nation's baby boomer's generation (those born between 1946 and 1964), a shortage of registered nurses is predicted to continue in the United States for years to come (American Association of Colleges of Nursing [AACNa], 2017). In order to meet the current and future workforce demands for competent nurses, schools of nursing are faced with the responsibility of increasing enrollments while still working to maintain rigorous academic standards. To further complicate this matter, the nursing profession is simultaneously facing a shortage of qualified faculty to teach and guide the education of future nurses. To meet the demands of increased enrollment, schools of nursing are currently hiring clinical experts who have no formal preparation in pedagogy or educational theory in order fill teaching vacancies (Anderson, 2009; Roberts, Chrisman, \& Flowers, 2013). Recruiting clinical experts (e.g., clinical nurse practitioners) to teach nursing students may contribute to alleviating the effects felt by the nursing faculty shortage, but it is not an ideal solution to the problem. Proficiency as a clinical nurse does not have a direct correlation with the ability to teach nursing students (Altmann, 2007; Stevens \& Duffy, 2017). The following sections will provide the reader with an understanding of the history and current state of nursing and nursing education that have contributed to this dilemma.

\section{Background}

In 1960, due to many factors, the United States experienced a shortage of trained physicians (Andreoli, 1987). Prior to 1969, the emphasis in graduate nursing education was on the preparation of graduate nurses for roles in the academic setting or nursing administration 
(Schoening, 2013). However, in 1969, in order to meet the demand for competent patient-care providers, the American Nurses Association (ANA), a national association with great influence on the profession of nursing and nursing education, advocated for the graduate preparation of nurse clinicians, such as nurse practitioners, and de-emphasized the importance of educating nurses for a role as nursing educators (Genrich \& Pappas, 1997). In the wake of this statement by the ANA, a monumental shift occurred in the curricular focus of graduate nursing education that lead to a dramatic increase in the number of nurses who were educated for the role of an advanced practice nurse, or clinical nurse practitioner (Andreoli, 1987). Subsequently, fewer graduate programs offered courses in nursing education as a primary area of study (Schoening, 2013). Due to the curricular change in graduate nursing programs, the profession of nursing "witnessed a dramatic decline in master's level [nursing] programs that prepare graduates for the faculty role" (Zungolo, 2004, p. 19). Proto and Dzurec (2009) captured this transition:

Several decades ago, graduate programs in nursing turned away from a focus on education, directing curricula instead towards clinical practice and administration. Teaching thus became a relatively invisible career option for nurses. Over time, efforts to market teaching roles diminished, further fostering a discrepancy between interest in positions in clinical and academic nursing. (p. 87)

Even though this change occurred many years ago, the profound effects of the ANA's position are still being felt in nursing education today.

\section{Nursing Faculty Shortage}

Due to a declining number of graduate programs available to prepare nursing educators, the nursing profession is now facing a profound faculty shortage (Dunham-Taylor, Lynn, Moore, McDaniel, \& Walker, 2007; National League of Nursing [NLN] Board of Governors, 2002). 
Zungolo (2004) described this phenomenon by stating "we are facing our most serious shortage of individuals who possess any formal knowledge about academic instruction or the organization and delivery of knowledge related to nursing practice” (p. 20). Unfortunately, Zungalo's statement is still true almost fifteen years later. According to a 2016-2017 report by AACN (2017b), there were over 1,567 full-time faculty vacancies identified in a survey of 812 schools with either baccalaureate or graduate nursing programs.

Several barriers have been identified as contributing factors to the faculty vacancies. These barriers include insufficient funds to hire new faculty and competition for other jobs by qualified faculty (AACN, 2016). The aging workforce of nursing educators is another factor that is contributing to nursing faculty vacancies. According to a recent study, the average age of a nursing educator is $\mathbf{5 7 . 8}$ years at the rank of a professor, 56.6 years for associate professor, and 50.9 years for assistant professor (AACN, 2017b). Considering the average age of retirement for a nursing faculty member is 62.5 years, the nursing faculty shortage will continue to plague the profession for many years to come (AACN, 2017b). Finally, an overwhelming factor in the nursing faculty shortage is the difficulty in recruiting qualified applicants for faculty positions and providing adequate educational preparation for teaching (AACN, 2016; National Advisory Council on Nurse Education and Practice [NACNEP], 2010).

\section{Nursing Shortage}

In addition to facing a shortage of qualified nursing faculty, the nursing profession is also facing a shortage of competent registered nurses. Unfortunately, like the nursing faculty shortage, the shortage of registered nurses is predicted to continue for many years. In the year 2000, the supply for registered nurses fell short of the demand by six percent and it is predicted that that percentage will increase to thirty-six percent by the year 2020 (NACNEP, 2010). The 
aging population is one reason for the increased need for qualified registered nurses in the United States. According to a United States Census Bureau report, in 2012 approximately 43 million residents were age 65 or older (Ortman, Velkoff, \& Hogan, 2014). However, by the year 2050, it is predicted that more than 83 million residents of the United States will be age 65 and older and in need of health care services (AACN, 2017b). One statistic projects the need for 649,100 replacement nurses in the workforce by 2025 to meet the demands of the aging population (AACN, 2017a).

As with nursing faculty, the aging registered nurse work force is another factor contributing to the need for qualified nurses. It is predicted that, within the next ten to fifteen years, more than one million registered nurses will reach retirement age and leave the profession (AACN, 2017a). The nursing faculty shortage is greatly affecting the number of competent registered nurses available to care for the citizens of the United States (AACN, 2017b). In a time where schools of nursing should focus on increasing their admission rates to meet the demand for qualified nurses, nursing schools are, rather, turning qualified students away and reducing admission rates due to the lack of qualified nursing faculty, classroom space, and clinical sites needed to accommodate large classes of students (AACN, 2017b). In 2016, nursing schools in the United States turned away 64,067 qualified applicants to both graduate and baccalaureate nursing programs (AACN, 2017a). The shortage of registered nurses may potentially have a severe impact on the safety and wellbeing of the public (Hinshaw, 2001).

\section{Current Efforts to Ease Nursing Shortage}

The issue of turning away qualified applicants to nursing schools, and therefore limiting the number of nurses available for the workforce, is not only being experienced nationally, but also locally. States, such as West Virginia, are enacting new legislation to meet the need for 
nursing faculty. In March of 2018, the Governor of West Virginia, Jim Justice, signed House Bill 4156 into action. According to section \$30-7-5A Schools of Nursing Faculty Requirements (2018), the state of West Virginia will now allow nurses to teach in schools of nursing as fulltime nursing faculty if they meet any of the following qualifications:

Have a graduate degree with a major in nursing, have a bachelor's degree in a major in nursing and be enrolled in a graduate program within one year of employment as a faculty member, or have a bachelor's degree with a major in nursing and at least fifteen years of direct patient care experience in nursing. (p. 2)

In addition, House Bill 4156 goes on to make the following provisions for nursing faculty with less than two years of educational experience: "The nursing program administrator will submit to the board mentoring and orientation plans as defined by the board guidelines under the guidance of a faculty member fully qualified in the specific teaching area and professional competence" (p. 3). The issue of nursing educators being unprepared to teach nursing students due to a lack of graduate preparation may become even more complicated. Under House Bill 4156, in the state of West Virginia, nursing faculty are not required to have completed graduate education in order to teach in a school of nursing. In light of the recent trend to hire clinical experts who are, perhaps, unprepared to teach undergraduate students, it is imperative to assure that future registered nurses who care for patients in West Virginia and beyond have been taught by competent clinical nursing educators.

\section{Purpose}

The purpose of the research study was to identify the unique clinical teaching practices and underlying knowledge of these teaching practices used by clinical nursing experts with no formal graduate preparation in education and who have transitioned into the role of the novice 
clinical nurse educators. Additionally, the grounding experiences of the teaching practices and how these teaching practices may change based on the clinical context was explored. Finally, areas for future professional development for clinical experts who transition into the role of a novice clinical nursing educator were identified. As the nursing and nursing faculty shortage continue in the United States, and even closer to home in West Virginia, the results of this study may be used to further inform nursing faculty and administrators within Schools of Nursing regarding the current teaching practices of novice clinical nursing educators. By exploring the teaching practices of novice clinical nursing educators, this investigation may provide empirical evidence to guide future research in the areas of mentoring or preparation of novice clinical nursing educators.

\section{Motivation for the Investigation}

In the fall of 2017 , I performed a pilot study to examine the teaching practices utilized by one novice nursing educator when teaching undergraduate nursing students in the clinical patient care area. The pilot study used ethnographic research methods to analyze data sources including: participant observation, ethnographic interviewing, semi-structured interviews, and document analysis. The novice nursing educator participant formerly worked as a licensed registered nurse for over 20 years and a certified nurse practitioner for over 17 year. She was considered to be a novice educator, as she had less than three years of teaching experience. Several findings were revealed from the pilot study. These included: when teaching in the clinical patient-care area the novice clinical nursing educator drew upon her experiences as a nursing student, asked primarily low level questions during the pre-and-post conference sessions, and utilized a teacher-centered approach, rather than a student-centered approach. These findings were used to inform areas of 
the literature review for this research study as well as to justify decisions pertaining to the research questions and methods of data collection and analysis.

\section{Research Questions}

Four clinical nursing experts who transitioned to the role of a novice nursing educator participated in this research study. Four overarching questions guided this research study:

1. What teaching practices and teacher knowledge do novice clinical nursing educators demonstrate or draw from when teaching undergraduate nursing students in the clinical patient-care area?

2. In what ways do novice clinical nursing educators change or adapt their teaching behaviors as a response to the context of the teaching/learning environment?

3. What grounding educational or professional experiences do novice clinical nursing educators refer to or draw upon when working in the role of the clinical nursing educator?

4. What are areas of teacher knowledge and/or teaching practices, utilized by novice clinical nursing educators, which preclude the facilitation of student learning and/or critical thinking?

\section{Significance of the Investigation}

Based on the statements and governmental actions presented in the background of this paper, it is clear that schools of nursing are exploring multiple ways to approach the nursing faculty shortage while continuing to provide competent and rigorous nursing education for the future nurses of West Virginia and beyond. These methods include utilizing nurse practitioners, nurse practitioner students, or even bachelor's degree prepared nurses with clinical expertise. However, these clinical experts lack the formal educational preparation in teaching and learning principles that are required to teach undergraduate nursing students (Kalensky \& Hande, 2017). 
Research on teaching practices used by nursing educators has often focused on the classroom or didactic area and not on the clinical teaching environment (Young \& Diekelmann, 2002). In fact, the identification of teaching styles used by clinical nursing educators to teach nursing students in the clinical patient-care area has not been widely explored, and there is a gap in the literature pertaining to what methods or practices clinical nursing educators are using to teach in the clinical setting (Hossein, Fatemah, Fatemeh, Katri \& Tahereh, 2010; Phillips \& Vinten, 2010). Additionally, Cangelosi, Crocker, and Sorrell (2009) argue that more research is needed in order to explore the role and perspectives of expert clinicians who have transitioned in to the role of a novice clinical educator. The research formerly conducted on the teaching practices primarily drew upon survey or participant interviews data (Scanlan, 2001). There is currently a lack of research studies drawing on observational data of in-the-moment teaching practices to describe the teaching practices used by novice clinical nursing educators.

This research study adds to the limited research available to the nursing education community by offering a detailed description of the teaching practices and distinct knowledge novice clinical nursing educators draw upon when teaching in the clinical patient care area. The findings of this study may also be used as a foundation for the development of continuing professional development for clinical nurse experts who transition into the role of a novice clinical nursing educator. Hutchings and Shulman (1999) use the term "the scholarship of teaching" to urge educators to conduct meaningful research that advances the profession and the practice of teaching (p. 5). Hutchings and Shulman (1999) argue "the scholarship of teaching can also make a place for "what" questions- those in which the task is not to "prove" but to describe and understand an important phenomenon more fully" (p. 9). The purpose of this study was to describe and make meaning of the teaching practices of novice clinical nursing educators in 
order to contribute to efforts to "advance the profession and the practice of teaching" (Hutchings \& Shulman, 1999, p. 5).

\section{Definition of Terms}

There are a number of terms that were used throughout this study. The following terms will be defined:

Academic nursing education. The process of facilitating learning through curriculum design, teaching, evaluation, advisement, and other activities undertaken by faculty in schools of nursing. Academic nursing education is a specialty area and an advanced practice role within professional nursing (NLN, 2012).

Clinical. "Clinical means involving the direct observation of a patient" (Gaberson, Oermann, \& Shellenbarger, 2015, p. 7).

Clinical Nurse Practitioner (CNP). According to the American Association of Nurse Practitioners (AANP) (2017), a nurse practitioner must complete a master's or doctoral degree program (AANP, 2017). Nurse practitioners diagnose and treat common acute illnesses and injuries, administer immunizations, conduct physical exams, manage chronic problems, and order lab services and x-rays (AACN, 2018b).

Clinical teaching or clinical instruction. "The central activity of the teacher in the clinical setting is clinical instruction or clinical teaching" (Gaberson et al., 2015, p.7).

Clinical teaching behaviors. "Those actions, displayed by clinical teachers in the field, that have been shown in the literature to affect student learning" (Mogan \& Warbinek, 1993, p. 161). Novice. According to Kumi-Yeboah and James (2012), a teacher with less than three years of experience is considered to be a novice. 
Pedagogy. The educational or instructional approach used to develop knowledge (Allan \& Smith, 2010). Pedagogy can refer to the nature of knowledge, how students learn, what is taught and how it is taught, and what is learning (Horsfall, Cleary, \& Hunt, 2012).

\section{Conclusion}

The United States, and more importantly, the state of West Virginia, is currently experiencing a nursing and nursing faculty shortage that is predicted to worsen in the foreseeable future. To meet the demand for nurses, nursing schools, both locally and nationally, are looking for ways to increase their student enrollments. Due to the lack of nursing educators who have educational preparation to teach students in both the classroom and the clinical patient-care area, schools of nursing have resorted to hiring clinical experts or nurse practitioners, with no educational preparation in teaching or pedagogy, to fill the gaps (Roberts, et al., 2013).

The purpose of this research study was to identify and describe the teaching practices and the grounding experiences of the teaching practices novice educators use when teaching undergraduate students in the clinical patient care area. This chapter provided an introduction to the research questions, purpose, and significance of the investigation. Chapter two will provide a review of the literature pertaining to the unique theoretical frameworks used to guide this investigation, the roles and responsibilities of the clinical nursing educator, and the lived experience of an expert nurse who transitions into the role of the novice clinical nursing educator. 


\section{Chapter Two}

\section{Literature Review}

\section{Introduction to the Literature Review}

The purpose of this literature review is to examine prior research, professional standards, as well as theories concerning nursing education in general and teaching as they relate to novice clinical nursing educators in particular. In order to provide the reader with a comprehensive understanding of the foundations of the phenomenon, drawing on the National League of Nursing's (NLN) (2012) Scope of Practice for Academic Nurse Educators, the review of the literature first examines the roles and responsibilities of the clinical nursing educator to facilitate student learning and he development of critical thinking in the patient-care area. This is followed by explicating Shulmans (1986) notion of Pedagogical Content Knowledge (PKC) and Ball, Thames, and Phelps (2008) framework of Mathematical Knowledge for Teaching. These frameworks are used to provide an understanding of the unique knowledge of a teacher. A review of the literature that examines the specific teaching practices that may be used in the clinical patient-care areas to develop students' critical thinking, knowledge, and skills is provided. The review ends by elaborating on the unique lived experience of the novice nursing educator in order to justify statements made claiming that clinical experts may be "unprepared" for the clinical nursing educator role (Anderson, 2009; Roberts et al., 2013). Benner's (2001) theory of Novice-to-Expert and Lave and Wegner's (1991) theory of situated learning provide the theoretical groundings for this section. This section also reviews literature related to the limited body of research that has been conducted pertaining to the grounding experiences of the teaching practices used by novice clinical nursing educators. 


\section{Preparation of a Nursing Educator}

According to the American Association of Colleges of Nurses (AACN), entry-level requirements to teach in an undergraduate nursing program are as follows: completion of a Bachelor's degree, completion of a Master's degree, an active registered nurse (RN) license, and nursing experience (AACN, 2018a; AACN, 2018b). Based on these requirements, there is no "official" pre-requisite that states nursing educators must have graduate education with a concentration in nursing education in order to teach undergraduate nursing students in the clinical patient-care area or in the classroom setting of a school of nursing. Graduate education for all master's prepared nurses, regardless of focus or specialty, is based on nine essentials of practice as defined by the AACN (2011) Essentials of Master's Education in Nursing. Although the core curriculum is the same for all graduate programs in nursing, where the graduate programs differ greatly is in the preparation of graduate nurses for specialty roles as either an academic nursing educator or a certified nurse practitioners (CNP). Registered nurses who had earned a master's degree, regardless of the focus of their graduate education, would meet the requirement to teach in an undergraduate nursing program. However, as the following sections will demonstrate, the graduate preparation of a nursing educator and that of a CNP are widely different.

To effectively perform in the role of the nursing educator, nurses must have pedagogical preparation in evaluation methods, curriculum development, and teaching strategies (Booth, Emerson, Hackney, \& Souter, 2016). Therefore, graduate programs that prepare nurses for an academic nurse educator role, ideally, include preparation in curriculum design and development, educational needs assessment, teaching methodologies, learner-centered theories and methods, and an in depth understanding of the discipline of nursing, nursing practice, and 
pedagogy (AACN, 2011). In addition, the academic nursing educator would have a supervised practice experience with a concentration in education (AACN, 2011). For an academic nursing educator, this supervised practice experience would provide an introduction to the role of the nursing educator, much like a student-teaching experience does for a pre-service teacher (Cooley \& De Gagne, 2015). Graduate education would then prepare the academic nursing education student to work within the scope of practice of an academic nursing educator.

Graduate course work for CNP's include content in: research, ethics, theoretical foundations of nursing practice, human diversity and social issues, health promotion and disease prevention, advanced health/ physical assessment, and professional role development including managing and negotiating heath care delivery systems, and management of client health/illness status (Commonwealth of Pennsylvania, 2018). Additionally, the advanced pharmacology course that CNP's are required to take includes information pertaining to principles of pharmacotherapeutics, pharmacokinetics, and prescribing medication (Commonwealth of Pennsylvania, 2018). Although CNP's have completed graduate education and hold a master's degree in nursing, the purpose of their graduate education was to facilitate the development of the clinical expertise needed to care for patients and not on the pedagogical practices to teach undergraduate students (Booth et al., 2016).

According to the National League of Nursing [NLN] Board of Governors (2017), "Nursing education is a specialized area of practice, and the nurse educators who understand and implement discipline-specific pedagogy are the vital link to a future workforce that will lead healthcare reform" (p. 2). Interventions to support the transition of clinical experts into an academic setting, such as mentoring and orientation programs, have been developed and utilized. However, these programs do not routinely focus on novice nursing educators' identification, 
development, or implementation of evidence-based teaching practices or pedagogy when teaching in the clinical patient care setting (Dunham-Taylor et.al. 2007).

\section{The Role of the Clinical Nursing Educator}

Shulman (2005) described the bedside teaching that occurs in the clinical patient-care area as the signature pedagogy of nursing education. According to Shulman (2005), signature pedagogies "are types of teaching that organize fundamental ways in which future practitioners are educated for their new profession" (p. 52). Clinical nursing educators face particular challenges in teaching nursing students, in that their pedagogies must not only measure up to the standards of teaching, but also to the standards of the nursing profession (Shulman, 2005). In clinical teaching, the clinical patient-care setting becomes the classroom where the teachinglearning process is centered on learning how to safely prove nursing care for a patient (Shulman, 2005). Shulman (2005) argues "signature pedagogies prefigure the cultures of professional work and provide the early socialization into the practices and values of the field" (p. 59).

\section{Signature Pedagogies}

Based on Shulman's definitions of signature pedagogies, one could argue that clinical education is the cornerstone of nursing education. Learning in a clinical setting "exposes students to the realities of professional practice that cannot be conveyed by a textbook or simulation" (Gaberson, et al., 2015, p. 9). In the clinical setting, the focus of the nursing students' learning is on the achievement of clinical competencies, as designated by the nursing programs' curriculum (Nielsen, 2009). Therefore, the one of the primary role of the clinical nursing educator is to facilitate learning in the clinical patient-care area that bridges the gap between the classroom and the clinical setting so that the student is able to work towards achieving the designated competencies (Dahlke, Baumbusch, Affleck, \& Kwon, 2012). It is also clinical nursing 
educator's responsibility to facilitate the development of their students' critical thinking when teaching in the clinical patient-care area (Gaberson et al., 2015). The following sections will provide the reader with information pertaining to the role and responsibilities of the clinical nursing educator to facilitate student learning in the patient-care area.

\section{Facilitation of Learning}

The term, "academic nurse educator" describes a teacher who fulfills an academic role as faculty in an academic setting (NLN, 2012). The Scope of Practice for Academic Nurse Educators (2012), published by the NLN, denotes the responsibilities of an academic nurse educator and "outlines the definition, historical perspective, values and beliefs, theoretical framework, scope of practice, and competencies or standards of practice of academic nursing education" (p.4). The NLN (2012) Scope of Practice for Academic Nurse Educators describes eight core competencies that define or delineate the responsibilities of an academic nurse educator. These are: facilitate learning, facilitate learner development and socialization, use assessment and evaluation strategies, participate in curriculum design and evaluation of program outcomes, function as a change agent and leader, pursue continuous quality improvement in the nurse educator role, engage in scholarship, and function within the educational environment.

\section{Perception of the Role of the Clinical Nursing Educator}

Although the role and responsibilities of a nursing educator have been clearly defined by the NLN, the educational background or preparation of a nursing educator may substantially contribute to the perception or understanding of their role and/or responsibilities when teaching in the patient-care area (Kelly, 2006). According to Kelly (2006), clinical nursing educators with graduate preparation in educational theories perceived that helping clinical nursing students relate underlying theory to practice in the clinical and asking questions in the clinical patient-care 
area to facilitate students' development of critical thinking skills were very important parts of their role. Conversely, nursing educators with a clinically-oriented master's degree viewed these two areas as a less important part of their role as a clinical nursing educator. Kelly (2006) contributed this difference to the clinically-focused faculty's expertise in the nursing care of the patient rather than on teaching. Based on these findings, Kelly (2006) suggested that clinical nursing educators need to be exposed to learning theories and teaching practices that are used to facilitate student learning in the clinical patient-care setting. The subject matter knowledge of a clinical nursing educator must transcend the subject matter knowledge of a clinical nurse in order to effectively facilitate learning in the clinical patient-care environment. The following section will provide a review of the literature focusing on the unique knowledge a teacher must possess in order to successfully fulfill this responsibility.

\section{Knowledge of a Teacher}

In order to facilitate nursing students' learning and development of critical thinking skills in the clinical patient-care area, a clinical nursing educator must have expertise in the "art of teaching” (Billings \& Halstead, 2009, p. 290). A teacher's talent lies in the ability to transform his or her expertise into a form that a student can comprehend or understand (Shulman, 1986). According to Shulman (1986), teachers "must be able to explain why a particular proposition is deemed warranted, why it is worth knowing, and how it relates to the other propositions, both within the discipline and without, both in theory and in practice" (p.9). To this point, Grossman, Schoenfeld and Lee (2005) stated the following:

Effective teachers know much more than their subjects, and more than 'good pedagogy.' They know how students tend to understand (and mis understand) their subjects; they know how to anticipate and diagnosis such misunderstandings; and they know how to 
deal with them when they arise. This type of knowledge is known as Pedagogical Content Knowledge. (p. 205)

Grossman et al.'s (2005) notion of Pedagogical Content Knowledge (PCK) is grounded in Shulman's (1987) definition: "the blending of content and pedagogy into an understanding of how particular topics, problems, or issues are organized, represented, and adapted to the interests and abilities of learners, and presented for instruction" (p. 8). PCK “entails the [teachers'] ability to transfer subject matter from ones' own knowing to another's knowing” (Fernandez-Balboa \& Stiehl, 1995, p. 294). PCK is composed of three broad categories: subject matter knowledge, general pedagogical knowledge, and contextual knowledge (Gess-Newsome \& Lederman, 2001). PCK is elaborated below as it relates to these three sections.

\section{Subject Matter Knowledge}

Subject matter knowledge is critical to the teaching-learning process (Grossman, 1990). For a teacher to have an understanding of subject matter knowledge, he or she must have an understanding of both the syntactic and substantive structures of a domain. Shulman (1986) argued that the syntactic structure includes "the ways in which truth or falsehood, validity, or invalidity are established" (p. 8). The substantive structure, according to Shulman (1986), includes "the variety of ways in which the basic concepts and principles of the discipline are organized to incorporate its facts" (p. 9). For a teacher to have a true understanding of subject matter, he or she must be able to translate or communicate why a particular topic or subject is important and how it relates to other topics within the discipline (Shulman, 1986). According to Shulman (1986):

The teacher need not only understand that something is so; the teacher must further understand why it is so...Moreover, we expect the teacher to understand why a given 
topic is particularly central to a discipline whereas another may be somewhat peripheral. This will be important in subsequent pedagogical judgments in regarding relative curricular emphasis. (p. 9)

Fernandez-Balboa and Stiehl (1995) suggested that, in higher education, individuals become teachers or professors based on their knowledge of subject matter and not because they "know how to teach it" (p. 295). Although knowledge of subject matter is an essential component of PCK, a competent educator cannot rely simply on personal knowledge of content as a basis for considering the teaching of others. Shulman (1986) argued:

Mere content knowledge is likely to be as useless pedagogically as content-free skills. We expect the teacher to understand why a given topic is particularly central to a discipline whereas another may be somewhat peripheral. This will be important in subsequent pedagogical judgments regarding relative curricular emphasis.” (p. 8) Simply put, having subject matter knowledge is not synonymous with knowing how to teach the subject matter in order to facilitate students' learning.

\section{General Pedagogical Knowledge}

A second component of PCK is General pedagogical knowledge (GPK). GPK coincides or overlaps with subject matter knowledge, as subject matter knowledge may inform a teacher's pedagogical considerations in how best to present or communicate the instructional content to students. Three distinct facets of GPK are: classroom management and organization, instructional models and strategies, and classroom communication and discourse (GessNewsome \& Lederman, 2001). A teachers' pedagogy includes the understanding of what is taught, how it is taught, the nature of how students learn, and how best to support students' learning (Horsfall et al., 2012). Although GPK, as defined by Shulman (1986), is inclusive of the 
classroom setting, teaching in the patient-care setting requires the clinical nursing educator to have knowledge of and use distinct pedagogies, instructional models, and teaching practices in order to facilitate student learning as well as the ability to safely manage students in the clinical patient-care area.

\section{Contextual Knowledge}

The final component of PCK, contextual knowledge, refers to the knowledge educators must have in order to adapt teaching practices to a specific setting or to meet the needs of a particular group of students (Grossman, 1990). In their exploration of clinical teachers, Pratt, Harris and Collins (2009) defined context in the following way: "Context is social as well as physical, involving a community of people working within norms and cultural conditions they have adapted as their own" (p. 135). The context in which clinical teaching occurs is a major determinant of its [teaching] effectiveness (Gaberson et al., 2015). In nursing education, contextual knowledge would refer to the level of student (sophomore, junior or senior), the required course and program student learning objectives, the environment in which teaching and learning occurs, and knowledge of what the student has been taught in previous nursing and science courses.

PCK is a multifaceted construct that may take teachers years to master. K-12 teachers may begin to develop their knowledge of PCK through a professional teacher education program and through teaching experience (Grossman et al., 2005; van Driel, Verloop, \& de Vos, 1998). A review of the literature shows that PCK is commonly used as a framework in many academic disciplines to examine and guide the preparation of teachers. These include: science education (van Driel et. al., 1998; Fraser, 2016; Loughran, Milroy, Berry, Gunstone, \& Mulhall, 2001; Smith \& Neale, 1989), technology education (Mishra \& Koehler, 2006; Rohaan, Taconis, \& 
Jochems, 2009), math education (Ball, Thames, \& Phelps, 2008; Hill, Ball \& Schilling, 2008;

Judson \& Leingang, 2016), and higher education (Fernandez-Balboa \& Stiehl, 1995). In preservice teacher education programs, PCK may be developed through observing other teachers, teaching in the classroom, and completing courses in teacher education (Grossman, 1990).

\section{Pedagogical Content Knowledge for Clinical Teaching}

Unfortunately, unlike K-12 teachers, the development of PCK for academic nursing educators may not begin until or unless they enroll in a master's of nursing program with a concentration in education. However, if a clinical nursing educator, like the participants in this study, did not receive instruction during their graduate program in education or pedagogical theories and practices, they may be unfamiliar with the essential elements and particular knowledge that comprises content knowledge for teaching and/or PCK. Limited studies have been conducted to examine and/or define the knowledge of a clinical teacher. As one example of this, Wolf, Beitz, Peters, and Wieland (2009) developed the Clinical Teaching Knowledge Test (CTKT), a 40-item multiple-choice test to examine the clinical nursing educators' knowledge of clinical teaching skills. Results of the study demonstrated that the main areas of weakness for the 160 participants was the use of educational theory in teaching in the clinical patient-care area. This study provides evidence that, in general, clinical nursing educators may lack the knowledge and underlying educational theory to effectively facilitate learning in the clinical patient-care area.

Three of the research questions in this study focused on particular teaching practices and underlying knowledge of novice clinical nursing educators. However, the nursing education literature lacks a framework that adequately describes the general pedagogical knowledge that is required of clinical nursing educators. Due to the fact that limited research has been done to 
examine the underlying knowledge of a clinical nursing educator, it was imperative to the work of this investigation that a framework was developed and used in order to understand the unique knowledge that clinical nursing educators draw upon and to identify the teaching practices derived from that knowledge. A review of the education literature demonstrated that the theoretical framework of Mathematical Knowledge for Teaching (Ball, Thames \& Phelps, 2008) provides a modern, structured, and organized framework that may be used to examine complex knowledge of subject matter and pedagogy that an educator must possess in order to facilitate students' learning. The following section introduces and describes each facet of the Mathematical Knowledge for Teaching framework. This framework was utilized in order to contextualize the facets of knowledge for clinical nursing educators.

\section{Mathematical Knowledge for Teaching}

In searching for a modern model to exemplify teaching knowledge for mathematic educators, educators Ball et al., (2008) transcended Shulman's (1986) principles of PCK when developing the practice-based theory of Mathematical Knowledge for Teaching framework (CKT). Hill, Ball, and Schilling (2008) describe Mathematical Knowledge for Teaching as teacher knowledge of the content that the students are supposed to learn. It is knowing how students think about specific content, knowing ways to unpack, represent, and make that content learnable. Finally, Mathematical Knowledge for Teaching involves knowing the ways to teach specific content. The Mathematical Knowledge for Teaching theoretical framework is divided into two sections, Subject Matter Knowledge and Pedagogical Content Knowledge. Each section is then further divided into three sections to demonstrate the unique qualities and knowledge of a skilled teacher. Figure 2.1, below, summarizes the constructs of the Mathematical Knowledge for Teaching framework. A review of each section follows. 


\section{Figure 2.1}

\section{Mathematical Knowledge for Teaching}

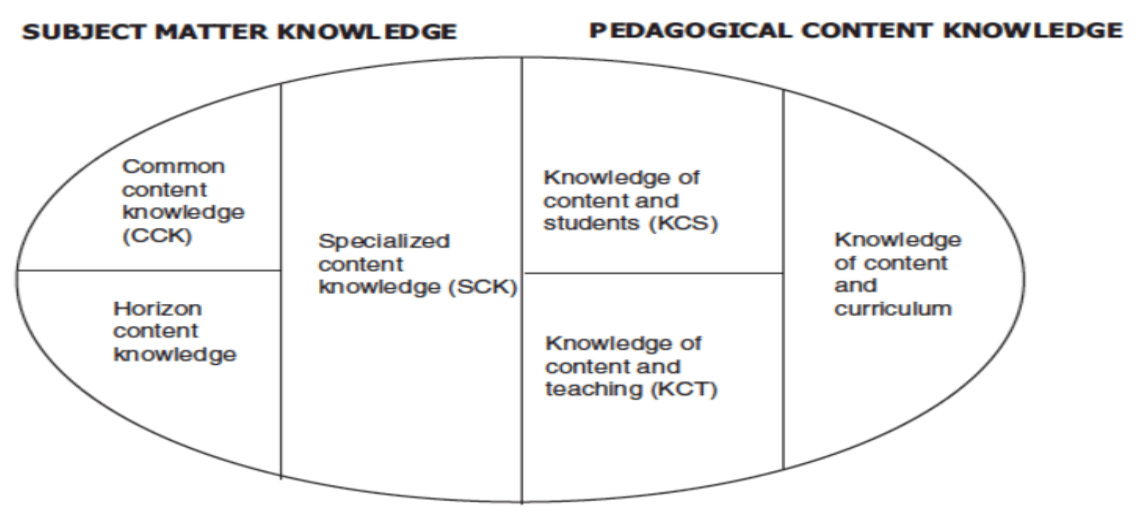

Note. Ball, Thames \& Phelps (2008) construct of Mathematical Knowledge for Teaching.

\section{Subject Matter Knowledge}

Within the Subject Matter Knowledge section, there are three sub-sections: Common Content Knowledge (CCK), Knowledge at the Mathematical Horizon, and Specialized Content Knowledge (SCK). Common Content Knowledge is described by Hill, Ball, and Shillings (2008) as "knowledge that is used in the work of teaching in ways in common with how it is used in many other professions or occupations that also use mathematics.” (p. 377). CCK involves recognizing when students provide incorrect information, understanding definitions common to the discipline, and the ability to do the tasks or know the information that they expect their students to know (Ball et al., 2008). CCK allows teachers to ask questions that may typically be answered by other individuals who know mathematics such as "what is a number that lies between 1.1 and 1.11" (Ball et al., 2008, p. 399). The knowledge needed to answer this question may not be particular to teaching, rather it may be used in a variety of settings and "not unique to teaching" (Ball et al., 2008, p. 399). CCK is the mathematical skill and knowledge that may be expected of any well-educated adult (Ball et. al., 2005). 
Like Common Content Knowledge, Specialized Content Knowledge (SCK) is based Shulman's (1986) original concept of subject matter knowledge (Hill et al., 2008). Ball et al. (2008) conceptualized Specialized Content Knowledge to mean the "mathematical knowledge that is not typically needed for purposes other than teaching" and "many of the everyday tasks of teaching are distinctive to this special work" (p. 400). SCK includes the skills and knowledge teachers draw upon that may be used to analyze errors, provide explanations as well as explicit examples of mathematical practices and languages (Ball et al., 2005). Examples of teaching tasks included in this realm of knowledge include: responding to students' why questions, giving explanations, choosing or modifying tasks to be easier or harder, and choosing and developing definitions (Ball et al., 2008).

CCK and SCK similar in that they are based on mathematical knowledge, and not knowledge of students or teaching (Hill et al. 2008). However, they differ in the way a teacher may use this knowledge in the academic setting. Common Content Knowledge allows the teacher to recognize correct or incorrect answers and facilitates the ability of the teacher to do the work that they assign to the students (Ball et al., 2005). However, mathematical Common Content Knowledge is not particular to math educators. Adults who have taken upper-level math courses may possess the same mathematical CCK as the math teacher. For example, this mathematical knowledge may be used by individuals to complete everyday tasks, such as to calculate the percentages of sales tax on an item. Conversely, Specialized Content Knowledge is not commonly used outside the realm of teaching and is beyond what is expected of an educated adult (Ball et al., 2005). It is distinctive to knowledge an educator must possess in order to perform the everyday tasks of teaching (Hill et al., 2008). Common and Specialized Content Knowledge go hand-in-hand to facilitate the teachers' deep understanding of, not only the 
content or information to be taught, but also with the knowledge to answer students questions, and connect one topic to another (Ball et al., 2008).

Finally, Horizon Content Knowledge (HCK) is described as: an orientation to an familiarity with the discipline (or disciplines) that contribute to the teaching of the school subject at hand, providing teachers with a sense for how the content being taught is situated in and connected to the broader disciplinary territory" (Jakobsen, Thames, Riberio \& Delaney, 2012, p. 4642).

A teachers' knowledge of the mathematical horizon may support them in making judgments about what is important to teach and orienting their instruction to the discipline (Jakobsen et al., 2012). HCK includes the teachers' awareness of how topics are related over the span of the curriculum (Ball et al., 2008). HCK allows teachers to make "sense of what students are saying and to act with an awareness of connections to topics that students' may or may not meet in the future" (Jakobsen, Thames, \& Riberio, 2013, p. 4). HCK is also inclusive of the mathematics teachers' ability to visualize how concepts learned early in a program may connect to mathematical concepts that are introduced in future courses (Ball et al., 2008). As an example of how a mathematics teacher may draw upon their HCK, .Ball et al. (2008) offer the instruction of a number line to third-grade students. At a third-grade level, the number line may look simple and not be a complex concept to master. However, the mathematics teacher will need to draw upon their knowledge of HCK in order to understand that the third-grade students will progress through grades four, five, and beyond, and will need to master additional concepts related to the number line, such as fractions and negative numbers, in order to plan instruction. In this way, teachers can create learning experiences that account for future possibilities in using the number line and, at the same time, can be careful to mitigate potential future misunderstandings. 


\section{Pedagogical Content Knowledge}

The second part of the Content Knowledge for Teaching Mathematics framework is associated with Shulman's (1986) original model of teacher knowledge and Pedagogical Content Knowledge (Ball et al., 2008; Hill et al., 2008). The three subsections are: Knowledge of Content and Students (KCS), Knowledge of Content and Teaching (KCT), and Knowledge of Content and Curriculum (KCC) (Ball et al., 2008; Hill et al., 2008). Knowledge of Content and Students (KCS) is a combination of knowledge that includes knowing about students and knowing about the discipline (Ball et al., 2008). Central to this construct is the teachers' knowledge of student conceptions and misconceptions, a teachers' ability to anticipate what a student may think or know, and what they may find confusing, and what examples to choose to facilitate a students' learning (Ball et al., 2008). KSC requires a teacher to have a deep understanding of not only the content they are teaching, but also how students may learn and/or come to understand the content.

Ball et al. (2005) offer the following example of KCS. A student is struggling to solve the problem $3+6=x+5$. The mathematics teacher may recognize that, based on the level of the learner, the student is struggling to solve the problem due to their misunderstanding of the symbol $\mathrm{x}$ or their misunderstanding of the use of the equal sign. Based on their KCS, the mathematics teacher may choose to use a different example or provide additional information and guidance in order to facilitate the students' understanding of the mathematical concept.

Knowledge of Content and Teaching (KCT) combines knowledge of the discipline and knowledge of teaching (Ball et al., 2008). This construct may include the following teacher responsibilities: designing instruction, choosing the sequence of content presentation or discussion, selecting of examples, choosing a particular teaching method, discriminating the 
advantages and disadvantages of using particular representations to teach a specific idea or concept. KCT requires an interaction between specific content understanding and an understanding of pedagogical methods that facilitate students' learning (Ball et al., 2008). For example, Ball et al. (2005) suggest that KCT includes the mathematics teacher' ability to place content for instruction in a sequence that is conducive to students learning. Mathematics teachers would draw from this knowledge when designing instruction to move from simple math concepts, such as simple addition of numbers, to more complex math concepts, such as division of fractions (Ball et al., 2008).

Knowledge of Content and Curriculum (KCC) is the final construct included in the Pedagogical Content Knowledge section. KCC may include a teacher's understanding of the school or course curriculum (Ball et al., 2008). Additionally, KCC may include the teachers' knowledge of what is to be taught and in what particular order it should be taught (Judson \& Leingang, 2016). Shulman (1986) suggests that a teachers' curricular knowledge encompass their understanding of not only the curriculum particular to their course, but also content that has been taught during the preceding semesters, during the concurrent semester, or will be taught in future semesters. For example, a second-grade math teacher would draw upon his or her Knowledge of Content and Curriculum related to math concepts introduced during the first-grade year, as prescribed by the school or school district, in order to plan instructional activities for his or her second-grade course.

Due to a lack of literature related to the unique knowledge and teaching practices of clinical nursing educators, and based on the a review of the literature, the Mathematical Knowledge for Teaching framework (Ball, 2008) was adapted and used as the theoretical framework during data collection and analysis in order to identify, define, describe, and analyze 
the in-the-moment teaching practices and knowledge of novice nursing educators. Table 2.1

describes each component of Ball et al.'s (2008) Mathematical Knowledge for Teaching model and how it was adapted to meet the constructs of clinical nursing education.

Table 2.1

Mathematical Knowledge for Teaching vs. Pedagogical and Subject Matter Knowledge for Clinical Nursing Educators.

\begin{tabular}{|c|c|c|}
\hline $\begin{array}{l}\text { Subject } \\
\text { Matter } \\
\text { Knowledge }\end{array}$ & Application to Mathematics & $\begin{array}{l}\text { Application to Clinical Nursing } \\
\text { Education }\end{array}$ \\
\hline $\begin{array}{l}\text { Common } \\
\text { Content } \\
\text { Knowledge }\end{array}$ & $\begin{array}{l}\text { Common content knowledge (CCK) is } \\
\text { described as "knowledge of a kind used } \\
\text { in a variety of settings- in other words, } \\
\text { not unique to teaching" (Ball et al., } \\
2008, \text { p. 399). } \\
\text { CCK involves recognizing when } \\
\text { students provide incorrect information, } \\
\text { understanding definitions common to } \\
\text { the discipline, and the ability to do the } \\
\text { tasks or know the information that they } \\
\text { expect their students to know (Ball et } \\
\text { al., 2008). }\end{array}$ & $\begin{array}{l}\text { Common content knowledge } \\
\text { (CCK) for the clinical nursing } \\
\text { educator includes knowledge } \\
\text { related to discipline of nursing. } \\
\text { This includes knowledge of disease } \\
\text { processes, nursing interventions, } \\
\text { and interpretation of laboratory } \\
\text { values. Additionally, this } \\
\text { knowledge is inclusive of the } \\
\text { clinical nursing educator ability to } \\
\text { apply his or her experience as a } \\
\text { clinical nurse to the patient-care } \\
\text { setting, including utilization of } \\
\text { clinical judgment and critical } \\
\text { thinking skills as they relate to the } \\
\text { acute care clinical setting. This } \\
\text { knowledge would have been } \\
\text { acquired during clinical nursing } \\
\text { educators' undergraduate or } \\
\text { graduate nursing education and/or } \\
\text { through clinical practice. }\end{array}$ \\
\hline $\begin{array}{l}\text { Specialized } \\
\text { Content } \\
\text { Knowledge }\end{array}$ & $\begin{array}{l}\text { Specialized content knowledge (SCK) } \\
\text { is based Shulman's (1986) original } \\
\text { concept of subject matter knowledge } \\
\text { (Hill, Ball, \& Schilling, 2008). Ball, } \\
\text { Hill, and Bass (2005) conceptualized } \\
\text { specialized content knowledge to mean } \\
\text { "the mathematical knowledge that } \\
\text { allows teachers to engage in particular } \\
\text { teaching tasks..." (p. 377). SCK is }\end{array}$ & $\begin{array}{l}\text { Specialized Content Knowledge } \\
\text { (SCK) is inclusive of specialized } \\
\text { knowledge that the clinical nursing } \\
\text { educator would need to utilize } \\
\text { when teaching nursing students to } \\
\text { care for patients in a clinical } \\
\text { patient-care area. This knowledge } \\
\text { would be specific to clinical unit } \\
\text { routines, the role of health care }\end{array}$ \\
\hline
\end{tabular}


particular to teaching, may be inclusive of everyday tasks, and is typically a knowledge set that is not needed or used outside the realm of teaching.
Horizon Content knowledge (HCK) is an awareness of how topics are related over the span, how topics relate to previous courses, how topics will relate to courses beyond. Horizon content knowledge is described as "an orientation to an familiarity with the discipline (or disciplines) that contribute to the teaching of the school subject at hand, providing teachers with a sense for how the content being taught is situated in and connected to the broader disciplinary territory" (Jakobsen, et al., 2012, p. 4642). A teachers' knowledge of the mathematical horizon may support them in making judgments about what is important to teach and orienting their instruction to the discipline (Jakobsen et al., 2012). professionals, and the role and responsibilities of the Registered Nurse working in a clinical patientcare area, and everyday task of the registered nurse including medication administration and documentation in the Electronic Medical Record (EMR). SCK also includes knowledge of the nursing process. The clinical nursing would have, ideally, acquired specialized content knowledge through clinical practice as a Registered Nurse. Horizon Content knowledge (HCK) involves the clinical nursing educators' knowledge of what content is important to each in the clinical patient-care area. It also includes the clinical nursing educators' knowledge of how the content taught in the clinical patient-area connects to the students' prior knowledge and future learning. Examples of this would include the clinical nursing educators' ability to engage the students' prior knowledge from general education courses, such as the sciences and of preceding nursing courses. HCK would include the clinical nursing educators' ability to relay information appropriate to the level of the learner. HCK would enable to the clinical educator to ask appropriate questions based on students' prior learning. Finally, HCK would enable the clinical nursing educator to scaffold content in the clinical setting based on their knowledge of the broader disciplinary learning needs.
Pedagogical Application to Mathematics

Content

Knowledge
Application to Clinical Nursing Education 
Knowledge of Content and Students

Knowledge of Content and Curriculum
Knowledge of Content and Students (KCS) is a combination of knowledge that includes knowing about students and knowing about the discipline (Ball et al., 2008). Central to this construct is the teachers' knowledge of student conceptions and misconceptions, a teachers' ability to anticipate what a student may think or know, and what they may find confusing, and what examples to choose to facilitate a students' learning (Ball et al., 2008). $\mathrm{KSC}$ requires a teacher to have a deep understanding of not only the content they are teaching, but also how students may learn and/or come to understand the content.

Knowledge of Content and Curriculum (KCC) includes knowledge includes a teacher's understanding of the school or course curriculum (Ball et al., 2008). HCK may also include the teachers' knowledge of what is to be taught and in what particular order it should be taught (Judson \& Leingang, 2016).
Knowledge of Content and Teaching
Knowledge of Content and Teaching (KCT) combines knowledge of the discipline and knowledge of teaching and strategies to guide student learning (Ball et al., 2008). This construct may include the following teacher responsibilities: designing instruction, choosing the sequence of content presentation or discussion, selecting of examples, choosing a particular teaching methods, discriminating the advantages and disadvantages of using particular representations to teach a
Knowledge of content and students (KCS) includes the clinical nursing educator's understanding how nursing students learn or come to understand concepts in the clinical patient-care area. This may include the selection of specific examples used in the patient care area in order to clarify or explain a concept. It also includes the clinical nursing educators' ability to anticipate what a nursing student may understand as well as common misconceptions. KCS may be used to help a student to understand how terms may be used in different ways on the clinical unit.

Knowledge of Content and Curriculum (KCC) includes the clinical nursing educators' knowledge of course and program objectives, clinical objectives, and clinical assignment objectives. Additionally, this would include the clinical nursing educators' knowledge of appropriate skills and nursing interventions the nursing student may perform or not perform independently or with supervision based on their level in the nursing program.

Knowledge of Content and Teaching (KCT) includes the clinical nursing educators' knowledge of specific teaching practices that facilitate learning and critical thinking in the clinical patient care area. These include the teaching practices of questioning, promoting discussion, active engagement, and role modeling. KCT also includes the clinical nursing educators' ability to choose the correct teaching method for in- 
specific idea or concept. KCT requires an interaction between specific content understanding and an understanding of pedagogical methods that facilitate students' learning (Ball et al., 2008). the-moment instruction of students. $\mathrm{KCT}$ for clinical nursing educators also incorporates constructs from Shulman's (1986) general pedagogical knowledge including classroom management and organization and classroom communication and discourse Gess-Newsome \& Lederman, 2001).

Note. Table constructed based on review of the literature.

\section{Clinical Teaching Practices}

To be an effective clinical nursing educator, it is important for the educator to have knowledge of nursing theory, pedagogy, and clinical nursing practice (Hanson \& Stenvig, 2008). Having the knowledge of and proficiency in utilizing clinical teaching practices is vital to facilitating nursing students' learning in the clinical patient-care area. However, the everchanging real-world patient-care environment may actually dictate what knowledge nursing students develop and which skills they master. The following sections provide literature related to effective teaching practices that may be used in the clinical patient-care area to promote learning and the development of critical thinking skills. Additionally, a discussion as to how teaching the real-world clinical context plays a role in the facilitation of learning in the clinical patient-care area.

\section{Development of Critical Thinking}

Although the NLN Scope of Practice (2012) does not discriminate between competencies of classroom educators and clinical nursing educators, Competency I: Facilitate Learning, may be applied to the distinct teaching strategies, methods, and practices to be used by the clinical nursing educator. According to Competency I: Facilitating Learning, the responsibilities of an academic nurse educator in facilitating learning include: creating opportunities for learners to 
develop their critical thinking and clinical reasoning skills and implementing a variety of teaching strategies and practices appropriate to learner needs, outcomes, context and content (NLN, 2012). The development of critical thinking skills is essential to a student nurse in order facilitate knowledge development and competent professional nursing practice (Facione \& Facione, 1996).

Kataoka-Yahiro and Saylor (1994) define the critical thinking process as it relates to nursing practice as" reflective and reasonable thinking about nursing problems without a single solution and is focused on deciding what to believe and do" (p. 352). To safely care for patients, nurses use critical thinking when developing clinical reasoning. Clinical reasoning is the process in which nurses analyze and evaluate patient information, such as the patient's presentation, past medical history, and prescribed medications, in order to make a clinical judgment (Gaberson et al., 2015). Tanner (2006) defined clinical judgment as "an interpretation or conclusion about a patient's needs, concerns, or health problems and/or the decision to take action (or not) or modify standard approaches, or improvise new ones as deemed appropriate by the patient's response" (p. 204). Clinical judgments are influenced by the situation, context, patient presentation, and culture of the clinical unit (Cappelletti, Engle, \& Prentice, 2014). Gaberson et al. (2015) argued "clinical learning activities must focus on developing students' critical thinking skills and dispositions throughout the nursing education program, so that students can build on their experiences to begin to refine these skills before they enter the professional workforce" ( $p$. 25). Therefore, the clinical nursing educator must have knowledge of and utilize teaching practices that facilitate nursing students' development of critical thinking skills in the clinical patient-care area setting (Dahlke et al., 2012). 


\section{Classroom vs. Clinical Teaching}

Promoting nursing students' development of critical thinking and clinical reasoning in the clinical area is very different from teaching in the classroom setting. In the classroom setting, the nursing educator may utilize lecture as a primary teaching strategy in order to correlate new and previously learned knowledge. In addition, in the classroom setting, the nursing educator has full authoity over the classroom enviroment, uses tests or quizzes to evaluate student learning, and develops interperstional relationships with the students enrolled in the course.

In comparision, in the clinical setting, the nursing educator is responsible for providing nursing students with real-life learning experiences and opportunities that facilitate a transfer of knowledge from the classroom to the clinical setting. This must be done without the luxury of relying on or referring to lecture notes, power-point slides, or outlines to guide them in their clinical teaching (Gaberson et al., 2015). Additionally, because the clincial nursing educator is teaching in the real-world setting of a healthcare facility, he or she has limited authority over the clincial enviroment (Karuhije, 1997). Also, in this setting, the clinical nursing educator is responsible for the supervison of the nursing students' provision of care on hospitalized patient. Karuhije (1997) argues "although classroom and clinical teaching share similarities, each role has unique differences requiring the acquisition of and ability to use a wide varity of contextspecific instructional, evaluative, and interpersonal abilities" (p.5).

\section{Clinical Pedagogies That Promote Critical Thinking}

According to the NLN Scope of Practice (2012) Competency I, Facilitating Learning, the academic nursing educator must implement a variety of teaching strategies or practices, grounded in educational theory, that foster's the transfer of students' knowledge from the classroom to the clinical setting (Billings \& Halstead, 2009; Karhuije, 1997). Research in this 
area has identified evidenced-based teaching practices, such as observing nursing students in practice, questioning, and conducting clinical conferencing, that may be used in the clinical setting to facilitate nursing students' development of critical thinking skills (Oermann, 1997; Twibell, Ryan, \& Hermiz, 2005). A further discussion of these as well as other clinical teaching practices used to promote nursing students' critical thinking and clinical reasoning is provided in the following sections.

\section{Facilitate Pre-and Post-Conference Discussion}

Engaging in group discussions or pre-conferences with nursing students at the beginning of the clinical day and post-conference discussions at the end of the clinical day is one teaching strategy clinical instructors may use in order to facilitate the development of students' critical thinking and clinical reasoning (Gaberson et al., 2014; Oermann, 1997; Twibell et al., 2005). Pre-and post-conferences are teaching practices that facilitate nursing students' critical thinking by allowing them to present and discuss rationales for patient-care decisions (Oermann, 1997). During the pre-conference, students are provided with opportunities to ask questions, seek clarification, and voice any concerns they may have in anticipation of caring for their assigned patient during the clinical day (Gaberson et al., 2014). Likewise, during post-conference sessions, students are able to reflect on the care they provided to their patient or patients during the clinical day (Oermann, 1997). Additionally, conducting clinical post-conferences allows students to benefit from listening to their peers describe their clinical day and experiences (Horsefall, 1990). Clinical conferences promote the development of critical thinking by providing the students with a safe space away from the patient-care area to present patient information to the clinical nursing educator and the clinical group in order to identify how or why difficult concepts are related to one another (Oermann, 1997). 
In order to conduct successful pre-and post-conference discussions, the clinical nursing educator is responsible for developing a plan for instruction, including selecting a topic for discussion, selecting a proper teaching method to facilitate the discussion, and a devising a method to evaluate student learning (Billings \& Hallstead, 2009). Discussion in pre- and postconferences should be an exchange in which, through asking open-ended questions and supporting learner responses, the clinical nursing educator encourages students to arrive at their own decision or to engage in self-assessment about their clinical care (Gaberson et al., 2015). By asking open-ended questions, the clinical nursing educator facilities student learning and the development of higher-level thinking skills (Billings \& Hallstead, 2009; Gaberson et al., 2015).

\section{Questioning}

Questioning is a teaching strategy that may be used during pre-and post-conference and during the clinical day to develop nursing students' critical thinking skills, facilitate discussion and learning, keep students engaged, and challenge students' thinking (Gaberson et al., 2015; Holland \& Ulrich, 2016; Nicholl \& Tracey, 2007; Merisier, Larue, \& Boyer, 2018). Several studies have been conducted to define the types of questions clinical nursing educators utilized in the clinical setting. Mogan and Warbinek (1994) developed the Observations of Nursing Teachers in the Clinical Setting (ONTICS) instrument in order to distinguish between and among, and categorize teaching practices of clinical nursing educators, including questioning, that either facilitated or presented a barrier to student learning. Giddings, Dyson, Entwistle, Macdiarmid, Marshall, and Simpson (2000) used the ONTICS tool (Mogan \& Warbinek, 1994) to develop the Checking out, Exploring, and Extending Questioning (CEEQ) model. This model identified three categories of questions used by clinical nursing educators. These are: checking out, exploring, and extending. "Checking out" questions included identifying the nursing 
students' level of knowledge, feelings, and their understanding of nursing actions. Types of questions used for this purpose included requesting, clarification, refocusing, and prompting. The nursing students' responses to these questions typically involved descriptive information sharing and knowledge recall. "Exploring" questions were used to elicit nursing students' prioritizations and reasoning nursing action, use of language when communicating with patients, and assessment. Sub-categories of questions included requesting explanations and posing problems. These types of questions required the nursing students to interpret, explain, and rationalize their thoughts or actions. "Extending thinking" questions prompted nursing students to reflect on their nursing practice, consider cause-and-effect of their actions, explore thinking processes, and consider the worth of the students' knowledge and skills. Sub-categories included reflective, hypothetical, and comparative. These types of questions were often used by clinical nursing educators to prompt or extend the discussion with the student or students.

Having knowledge and understanding of different level and types of questions enables the clinical nursing educator to ask an array of questions, including high-level or clarifying questioning, in order to facilitate student learning and promote critical thinking (Oermann, 1997). Although the types of questions asked in the clinical setting may be used as a tool to facilitate learning, research suggests that, in the clinical setting, clinical nursing educators tend to ask nursing students low-level knowledge questions rather than high-level critical thinking questions (Giddings et al., 2000; Sellappah, Hussey, Blackmore, \& McMurray, 1998). The level of education and teaching experience may actually be a factor that affects clinical nursing educators' ability to ask high-level questions (Phillips \& Duke, 2001). In fact, Phillips and Duke (2001) found experienced nursing educators are more likely to ask higher-level thinking 
questions compared to educators with less teaching experience and no formal education in pedagogy.

\section{Role Modeling}

In addition to facilitating learning through conducting pre-and-post conferences and discussion, role modeling is a teaching strategy used by clinical nursing educators to facilitate learning in the clinical patient-care area. The concept of role modeling is based on the observational learning construct of Bandura's (1977) social learning theory, in which people learn to organize and model practices based on observation (Bethards, 2014). Through role modeling, the clinical nursing educator provides verbal and non-verbal cues or professional behaviors that nursing students can imitate when caring for patients (Sander \& Welk, 2005). A clinical nursing educator may use role modeling as an effective teaching strategy in the clinical patient-care area in order to teach a variety of psychomotor and technical skills, "soft" skills, such as methods of communication and conveying empathy, as well as to promote the emotional and spiritual development that they will need to function as a practicing nurse (Gaberson et al., 2015; Nouri, Ebadi, Alhani, Rejeh, \& Ahmadizadeh, 2013). Nouri et al., (2013) found that using role modeling as a teaching strategy promotes emotional development, spiritual development, and intellectual development of nursing students, including the promotion of active learning and critical thinking.

According to the NLN Scope of Practice (2012) Competency 1, the clinical nursing educator is responsible for implementing a variety of teaching strategies and practices appropriate to learner needs, outcomes, context and content. As the research studies by Nori et al., (2013), Phillips and Duke (2001), Sellappah et al. (1998) and others demonstrate, clinical conferences, questioning, and role modeling are examples of the variety of teaching practices 
that clinical nursing educators use in the clinical area to facilitate the development of nursing students' critical thinking. The findings of these studies, however, are not particular to the teaching practices employed by clinical nursing experts who have transitioned into the role of the novice clinical nursing educator. Additional research in this area is needed in order to investigate the teaching unique practices used by novice clinical nursing educators. The results of this investigation may contribute to this gap in the nursing research.

\section{Clinical Context and the Learner}

Teaching in the clinical patient-care area requires the ability of the clinical educator to teach in short periods of time and to make points memorable so that learners can retain them (Irby, 2014). In the clinical patient care area, the primary role of the student nurse is that of learner, not of nurse, and providing care for a patient does not automatically translate into student learning (Gaberson, et. al., 2014). Therefore, in the clinical setting, the clinical nursing educator should not focus solely on teaching the nursing student how to provide care to a patient. Teaching should also, however, focus on the students' development of the essential cognitive, psychomotor, and affective skills that are core outcomes of clinical education in undergraduate nursing programs (Oermann \& Gaberson, 2017). As the following sections will demonstrate, teaching students in the context of the fast-paced, highly-demanding real-world clinical setting can present a challenge. Therefore, the utilization of specific teaching practices may be dictated by the context of the real-world learning environment.

\section{Clinical Context}

Hoffman and Donaldson (2004) examined how the context of the unpredictable realworld clinical environment influenced the clinical teaching in an academic medical center in the United States. The results of the investigation identified several tensions that influenced clinical 
teaching practices and actions used by the clinical educators including type of illness, number of patients, and medical needs of the patients influenced the types of teaching practices used during the teaching session. The authors (2004) state "the higher the volume the less teaching takes place and that is because more time needed to be devoted to making sure the patients are getting taken care of" (p. 450). Conversely, during times when the team rounded on fewer patients and/or patients with fewer medical needs, the clinical instructor was able to use teaching practices that facilitated the medical students' in-depth exploration of the patient condition.

\section{Learning Opportunities}

The context of the patient-care environment can also influence the types of learning opportunities and time-spent on-task in the clinical patient-care area. Limited studies have investigated the in-the-moment teaching practices of clinical nursing educators in the clinical patient-care setting. Hsu (2006), Ironside, McNelis, and Ebright (2014) found that interactions between the student and the clinical nursing educator focused more on tasks, such as assuring the student was doing their assigned work, such as changing dressings, and assisting the student in medication administration. Little time was devoted to nursing students' development of critical thinking or problem solving skills. Evaluation of the students' knowledge was limited to basic knowledge of medications and laboratory values. The educators, therefore, allotted less time to evaluating the student's overall understanding of higher-level concepts and engaging in activities to promote problem solving and critical thinking abilities. The nursing students were very focused on implementing the assigned tasks for their patient, such as completing activities of daily living (ADL's), in a timely manner rather than seeking out new learning opportunities on the clinical unit. Although the acuity level (e.g. how much nursing care was required) of the patient cared for by the students and clinical nursing educators in these studies was not reported, 
these investigations provide evidence that, when teaching in the "real-world" patient-care area, clinical nursing educators may prioritize skill or task completion over the use of questioning or other teaching practices in order to meet the clinical needs of the patients.

\section{Developmental Level of the Learner}

Clinical teaching practices may also differ based on the students' developmental level (Conigliaro \& Stratton, 2010). Gardner and Harrelson (2002) suggest that, as the learner advances in competence, skills, and knowledge, the method of instruction or instructional styles should also change. For example, a clinical nursing educator may use a directional teaching method with novice clinical students, such as with sophomore level nursing students. However, when teaching a more experienced learner, such as with senior level nursing students, the clinical educator may use delegation as an instructional teaching method.

The review of the research on clinical teaching demonstrates that clinical teaching practices may be influenced or dictated by not only the level of the learner, but also the realworld patient-care environment or context. Unfortunately, no research study has been conducted to examine how the context of the learning environment may affect the teaching practices of clinical nursing experts that have transitioned into a novice clinical educators. For this reason, one of the research questions of this investigations examines how teaching practices of novice clinical nursing educator change in context in order to inform this area of nursing education research.

\section{Nursing Students' Perspective on Clinical Teaching}

The purpose of this research study was not investigate the nursing students' perspectives of the positive and negative teaching practices demonstrated by novice clinical nursing educators. However, the nursing students' perspective of clinical teaching practices that both 
promote and inhibit the facilitation of learning in the clinical patient-care area is important to consider. The review of the literature that follows provides an understanding of nursing students' perspectives in order to inform and understand interactions between the educator and the student in the clinical patient-care area.

Several behaviors were identified by nursing students as positive attributes of a clinical nursing educator. For example, the ability to communicate expectations for student learning or clinical performance was rated as one of the most important qualities of an effective clinical nursing educator by nursing students (Arkan, Ordin, \& Yilmaz, 2018; Hanson \& Stenvig, 2008; Lundin \& Futhullah, 2016; Sweet \& Broadbent, 2017). Being approachable, positive, supportive, and helpful to the nursing students are also important clinical teaching practices of an effective nursing instructor in order to facilitate learning in the clinical environment (Beitz \& Weiland, 2005; Lundin \& Futhullah, 2016; Sweet \& Broadbent, 2017). Other positive attributes of an effective clinical nursing instructor include: providing motivation for learning, demonstrating an empathetic, kind, and/or respectful attitude when working with nursing students, encouraging discussion, and providing positive reinforcement and encouragement to nursing students when learning in the clinical patient-care area (Hanson \& Stenvig, 2008; Lundin \& Futhullah, 2016; Sweet \& Broadbent; 2017; Valiee, Moridi \& Garibi, 2016).

Several behaviors were identified by nursing students that were perceived as barriers to learning in the clinical patient care area. For example, nursing students learning in the clinical patient-care area may be affected by the clinical nurse educators' lack of familiarity with the patient population, clinical incompetence, or lack of confidence in their teaching abilities (Killam \& Heerschap, 2013; Melincavage, 2011). Additionally, nursing students' perception that the clinical educator is exhibiting a lack of dedication or commitment to student learning, or "just do 
it [teaching] for the money" may also inhibit student learning in the clinical patient care area (Killam \& Heerschap, 2013, p.687). Another barrier to learning included nursing students' perceptions that their clinical nursing educator served more in the role of the evaluator rather than in the role of a teacher (Sharif \& Masoumi, 2005). Finally, poor or inadequate communication, asking too many questions in front of a patient, maintaining unattainable expectations, and seeking out and exploiting deficiencies of students' knowledge were all found to be barriers to student learning in the clinical patient-care area (Arkan et al., 2018).

\section{The Novice Clinical Nursing Educator}

As this review of the literature has demonstrated, the clinical nursing educator requires a working knowledge of aspects of knowledge of the learner, knowledge of context, knowledge of pedagogically sound teaching practices, subject matter knowledge, and subject matter knowledge for teaching (Shulman, 1996). According to Karuhije (1986) "a clinical teacher needs to know how to teach" and clinical teaching "is far too important to be delegated to the least experienced or least prepared" (p. 143). This section of the review is devoted to exploring literature related to the clinical nursing expert's transition to a novice clinical nursing educator. Benner's (2001) Novice-to-Expert framework is used to understand the unique developmental differences between a novice and an expert. Additionally, Lave and Wegner's (1991) theory of Situated Learning, along with theories of legitimate peripheral participation and communities of practice, are explored to provide an understanding of how novice nursing educators may adapt to learning to teach "on the job." The grounding experiences that novice clinical nursing educators often draw upon will be explored. Finally, a review of the literature of the unique lived experience of the transition of a clinical expert to the role of a novice clinical nursing educator is provided to in 
order to gain an understanding and appreciation for the stressors and emotions novice clinical nursing educators may experience while learning to teach on-the job.

\section{Novice-to-Expert Model}

Cangelosi, Crocker and Sorrell (2009) coined the phrase, "expert to novice" to describe the unique phenomenon of an expert clinical nurses who transition into the role of novice nursing educators (p. 386). The phrase "expert to novice" is a play on words of Benner's (2001) Noviceto-Expert theoretical model. Benner's (2001) model is used to examine the lived experience of the 'expert-to-novice' or the expert clinical nurse who transitions to the role of the novice clinical educator. Benner (2001) developed the Novice-to-Expert model based on the Dreyfus (1986) Model of Skill Acquisition. Key to the Dreyfus model is the process by which, through experience, learners move through five stages of development as they transition from novice to expert. These five stages of development are: (a) novice, (b) advanced beginner, (c) competent, (d) proficient, and (e) expert (Dreyfus, 2004). Benner applied the Dreyfus (1986) model to nursing practice based on her observations of fifty-one experienced nurses, eleven new graduate nurses, and five senior-level nursing students as they provided nursing care to patients in several different hospitals. By generalizing the Dreyfus (1986) Model, Benner (2001) applied the same five stages of development to nursing practice in order to understand the specific skills and clinical knowledge a nurse must possess to transition from a novice to an expert nurse (Altmann, 2007; Benner, 2001). The characteristics and/or practices of each of the five stages of development of professional nurses are elaborated below in

\section{Table 2.2}

Characteristics and Behaviors of the Five Stages of Development of Professional Nurses

Stage of Characteristics and/or Behaviors




\begin{tabular}{ll}
\hline Novice & A novice nurse has no previous experience of the situation in which he or she is placed, and \\
the behavior of the novice is extremely inflexible and limited. Because they lack experience, \\
novice nurses must be given context-free rules or guidelines to govern their performance. \\
The title of "novice" is not just limited to a nursing student or new graduate nurse. Rather, \\
the title of novice can be given to any nurse entering into a setting where he or she has no \\
previous experience (Benner, 2001; Dreyfus \& Dreyfus, 1986). \\
\hline Advanced & Going from a novice to an advanced-beginner stage only occurs through considerable \\
beginner & experience with real situations. Advanced beginners demonstrate marginally acceptable \\
& performances and begin to make decisions based on experience, not on established rules or \\
& instruction (Benner, 2001; Dall'Alba \& Sanberg, 2006; Dreyfus \& Dreyfus, 1986; \\
& Ramsburg \& Childress, 2012). \\
\hline Competent & To reach the stage of competence, a nurse must work in a role up to three years and he or \\
& she must be able to develop a plan for the particular situation in which they are placed and \\
& be able to make decisions based considerable analytic thought. Competent nurses are able to \\
& see how their own actions contribute to long-range goals or actions (Benner, 2001; \\
Ramsburg \& Childress, 2012). & A proficient nurse is able to see the situation as a whole, rather than in separate parts, and \\
acts based on experience. The proficient nurse is able to determine what is normal and what \\
is abnormal in their patients' presentation (Benner, 2001; Dall'Alba \& Sanberg, 2006; \\
Dreyfus \& Dreyfus, 1986; Ramsburg \& Childress, 2012). \\
\hline Proficient \\
Clinical practice for an expert nurse is second nature. An expert nurse no longer requires or \\
relies on rules or guidelines to govern their practice. Instead, when making clinical \\
decisions, they now draw upon their years of nursing experience, intuition, background, and \\
a deep understanding of the situation. Their performance is now flexible, fluid, and highly \\
proficient. They recognize a problem and simultaneously know how to solve it (Altmann, \\
2007).
\end{tabular}

According to Benner (2004), nurses may be at different levels of skills in different areas of practice based on their background, skills, and knowledge. For example, a nurse who has many years of experience taking care of adult patients would be considered an expert in caring for a 56-year old patient, but would be considered a novice when caring for a premature baby or young child (Benner, 2004). This concept of a professional nurse simultaneously functioning in the role of an expert and a novice correlates with the work of this study. Based on Benner's (2001) theory, the expert clinical nurse would be considered an expert when prescribing medications or completing a detailed health history for a patient, but, due to their lack of teaching experience, would be considered a novice when working in the role of a clinical nursing educator. 
When transitioning from an expert clinical nurse to a novice nursing educator, the once so familiar patient care environment where the nurse was the expert in planning, implementing, and evaluating nursing care of patients now becomes an unfamiliar and, perhaps, daunting teaching environment. According to Benner (2001) the behavior of the novice clinical nursing educator may be extremely inflexible and limited. Novice clinical educators must utilize contextfree rules or guidelines to govern their teaching practice. In the clinical teaching setting, the novice clinical educator takes on a new role and new responsibilities. Unfortunately, according to Cangelosi et al. (2009), proficiency as a nurse does not translate into proficiency as a clinical nursing educator. Because of a lack of graduate education with a concentration on learning theories and teaching practices and teaching experience, the novice clinical nursing educators may have to learn to teach "on-the-job."

\section{Situated Learning}

The theory of Situated Learning was used in this investigation in order to understand the unique experiences of novice clinical nursing educators as well as to gain an understanding of how novice clinical educators learn to cope with and adapt to the challenges of learning to teach “on-the- job." Lave and Wegner (1991) proposed that, when learning "on the job”, or through a situated activity, learning is facilitated through the interaction with the real-world environment. This "real-world" environment may include interaction with equipment, people, and/or through completing the required task for the role (Lave, 1989). Lave and Wenger (1991) suggest that situated learning has four interrelated aspects which include: learning is best situated in the context of authentic practice, learning can be transferred to similar situations, learning is primarily a social process, and the learners' prior knowledge is essential to the formation of new knowledge. Additionally, Brown, Collins, and Duguid (1989) conclude that what is being 
learned cannot be separated from the environment or context in which it is being learned.

Situated Learning theory implies that the learning or professional development of a teacher, or, in the case of this study, the novice clinical nursing educator, needs to be organized around tasks that the novice educator will need to accomplish in the clinical setting and, therefore, is inseparable from the context in which teaching-learning occurs (Szmanski \& Morrell, 2009). Simply put, a teacher cannot learn to teach unless they act in the role of a teacher.

\section{Legitimate Peripheral Participation}

Lave and Wenger (1991) use the term legitimate peripheral participation to describe the process of "newcomers to move towards full participation in the sociocultural practices of a community" (p. 29). The principle of legitimate peripheral participation implies that a novice learner may begin the learning process by first observing, and then performing basic tasks. It is through active participation with people within the working environment that the novice has joined, the novice learner will become more skilled and knowledgeable, take on more responsibilities, and, eventually acquire the skills, values, and norms of the community of practice (Mann, 2011).

Several diverse disciplines, including clinical educators from the field of medicine (Dornan, Boshuizen, Kng and Scherpbier, 2007; MacDougall \& Drummond, 2005; Mann, 2011) medical laboratory science (Miller, 2014), technology (Szymanski \& Morell, 2009), nursing research (Gieselman, Stark \& Farruggia, 2000), adult education (Black \& Schell, 1995) and professional teachers (Thacker, 2015), have used theories and concepts of situated learning or legitimate peripheral participation as a foundational framework for their research. The field of medical education has also examined Situational Learning theory as a framework for developing both medical students, and future medical educators. MacDougall and Drummond (2005) found 
that, like the novice nursing educators in this study, medical doctors "are experts in what they teach [however] most have little or no training in how they teach" (p. 1213). Mann (2011) argued "situational learning offers an integrative theoretical perspective within which to conceptualize learners and learning, develop and test new approaches, and realize our mutual goal of preparing learners [and eventual medical educators] effectively as members of the medical profession" ( $p$. 67).

\section{Communities of Practice}

The concepts of situated learning and legitimate peripheral participation are based on the idea that learning is always tied to context and social constructs and occurs through participation in the activities of a community (Mann, 2011). Communities of practice, a concept that is often aligned with situated learning theory, is central to knowledge development of "newcomers", or novice learners. Lave (1996) argues "learning is a facet of the communities of practice of which they are composed." (p. 150).

Drawing upon Lave \& Wenger's (1991) concept of professionals learning in a community of practice, Miller (2014) investigated medical laboratory science clinical educators' beliefs about teaching and how they view themselves as clinical educators. Miller (2014) argued that "clinical instructors are also associated with more than one community of practice.

Generally, their primary identification is with their professional discipline and not their educational role" (p. 98). Findings of this study (2015) demonstrated that expert clinicians who transition into the role of the clinical educator may struggle in identifying in their role as a teacher. 


\section{Challenges to Learning to Teach On-the-Job}

Based on the theories of situated learning, legitimate peripheral participation, and communities of practice, as a novice nursing educator advances through Benner's (2001) stages of development, he or she would begin to develop concepts of teaching practices through "onthe-job" experience. The novice clinical nursing educator would first learn their role and responsibilities through observation, and then by performing in the role educator. Unfortunately, unlike medical education, nursing education does not often draw upon the practical application of the theories of legitimate peripheral participation or communities of practice when introducing novice clinical nursing educators to the role of the teacher.

An additional challenge to novice clinical nursing educators may include a lack of guidance from mentors or senior faculty (Cooley \& De Gange, 2016). Mentoring and new faculty orientation programs have primarily been developed and implemented to introduce and integrate new faculty to the role of the academic nursing educator (Shanta, Kalaneck, Moulton, \& Lang, 2011). However, not all novice educators are assigned a mentor upon hire (Cangelosi et al., 2009; Morin \& Ashton, 2004). To compound this issue, mentoring or orientation programs offered by schools of nursing may not fully meet the needs of novice clinical nursing educators (Genrich \& Pappas, 1997; Roberts et al., 2012).

Regardless of educational preparation or experience of the educator, when assuming the role of a clinical nursing educator, administrators of nursing schools expectations of minimum competencies for a novice nursing educator to include "the ability of the clinical nursing educator to facilitate student learning, development, and socialization into the nursing profession" (Poindexter, 2013, p. 563). Therefore, upon hire, novice clinical nursing educators may be expected to meet the same clinical teaching responsibilities as more experienced clinical 
nursing educators. Likewise, they may not have the opportunity to observe other teachers teaching in the clinical setting prior to assuming the role of the clinical teacher. In fact, prior to assuming the role and responsibilities of the clinical nursing educator, the only exposure the novice may have had to the role of a clinical nursing educator was during their experience as an undergraduate or graduate nursing student.

Insufficient teaching experience and/or a lack of an educational background in teaching, may present significant challenges to a clinical nursing expert who assumes the role of the clinical nursing educator (Anderson, 2009; Anibas, Brenner, \& Zorn, 2009). These day-to-day challenges may include: working to teach students critical thinking, managing the instruction of multiple students at one time, managing student issues, evaluating student performance, and managing students' emotional behaviors. Other challenges may include: forming relationships with students, understanding the unique language of academia, facing a lack of confidence in their teaching skills and abilities, and insufficient time to perform teaching tasks (Anderson, 2009; Brown \& Sorrell, 2017; Cooley \& De Gagne, 2016).

\section{Emotional challenges}

Due to these on-the-job challenges, clinical nursing experts may experience emotional or social challenges as they assume the role and responsibilities of a novice nursing educator. Considering Benner's (2001) theory of novice-to-expert, when making a career change, the clinical nursing expert would experience a role transition requiring them to develop a new identity and become accustomed to the values and norms of an academic educator (Anderson, 2009). Meleis (2010) describes role transition as "a change in role relationships, expectations, or abilities. Role transitions require the person to incorporate new knowledge, alter his behavior, and thus change his definition of himself in his social context" (p. 15). Prior to transitioning to a 
novice educator, the clinical nursing expert may have experienced security and comfort in the role of an expert, but that safety and security is removed when taking on the role of educator (Anderson, 2009). Role transition may lead to feelings of uncertainty or instability as one moves into an unfamiliar role (Meleis, 2010). These feelings may manifest themselves in several emotions including grief, powerlessness, anxiety, depression, or mourning (Meleis, 2010).

\section{Role Strain}

Novice educators may also experience role stress or role insufficiency during their transition. Role stress may cause the novice nursing educator to experience role strain, or an emotional response to the stress, which may manifest in feelings of isolation and being overwhelmed by the responsibilities of the new teaching role (Clark, 2013). Role insufficiency is "any difficulty in the cognizance and/or performance of a role or of the sentiments and goals associated with the role behavior as perceived by the self or by significant others and may result from the lack of knowledge of what behaviors or goals the role demands" (Meleis, 2010, p. 16). Novice nursing faculty often experienced stress due to not having knowledge of the educational theories or background experience that is required of their new role or accepting a faculty position without fully understanding the role of an academic nursing educator (Anibas, et al., 2009; Mann \& De Gange, 2017; Brown \& Sorrell, 2017; Weidman, 2013; Anderson, 2009).

\section{Role Ambiguity}

Role ambiguity may be experienced by novice nursing educators when transitioning into their new role due to a lack of socialization to the academic culture (AACN, 2018a; Clark, Houten, \& Perea-Ryan, 2010; Owens, 2018). Role ambiguity occurs when the role and responsibilities assumed by novice nursing educator lack clarity or are not well defined (AACN, 2018a). When teaching students in the clinical patient-care area, the focus of a clinical educator 
should be "teaching not doing" (Gaberson et al., 2015, p. 16). Novice nursing educators may be very torn between assuming the role of the clinical nurse providing patient care, which would be second nature, and stepping back to facilitate the nursing students' learning experience (Paul, 2015).

\section{Facing the Challenge of Learning to Teach On-the-Job}

In order to meet these day-to-day challenges and adapt to the stressors associated with on-the-job teaching, novice clinical nursing educators develop coping mechanisms that may include drawing upon personal and professional experiences in which to ground their teaching. Without the knowledge of current evidence-based teaching practices, pedagogy, or learning theories, novice educators may resort to teaching as they were taught in their undergraduate or graduate programs, rather than according to evidenced-based teaching practices (Anibas et al., 2009; Krisman-Scott, Kershbaumer, \& Thompson, 1998; Mann and De Gagne, 2017; Owens, 2018; Shanta et al., 2011). Additionally, in order to cope with these challenges, novice nursing educators may also emulate teaching practices, such as role modeling and questioning, that are similar to those they had witnessed being used by experienced clinical educator peers (Phillips \& Vinten, 2010; Scanlan, 2001; Schriner, 2007). Finally, without previous teaching experience, novice nursing educators often use personal life experiences, graduate education, teaching skills developed for patient-education while employed as a clinical nurse as preparation for their role as a nursing educator (Anibas, et al., 2009; Mann and De Gagne, 2017; Schoenig, 2013). One of the purposes of this research study was to examine the grounding experiences of clinical nursing experts who transition into the role of the novice clinical nursing educator. The results investigation will contribute to the limited research in this area on this particular population. 


\section{Conclusion}

In conclusion, "knowing the practice field [of nursing] is not the same has knowing how to teach in the field" (Bastable, 2014, p. 10). Roberts et al. (2013) and Poindexter (2013) argue that the preparation of competent nurses must be facilitated by competent and qualified nursing educators to avoid compromising the quality and integrity of the nursing profession. Therefore, ideally, clinical nursing educators would have knowledge of and utilize research-based pedagogies, teaching practices such as conducting pre-and post-conferences, role modeling, and questioning, in order to promote nursing students development of critical thinking and clinical reasoning in the clinical patient-care area. Also, it is important for a clinical nursing educator to have a contextual understanding of the learning environment as well as an understanding of how best to negotiate the fine balance between providing competent care to the patient and facilitating nursing students' learning in the patient-care area. According to the NLN (2012), teaching and learning strategies need to be innovative, varied, and evidence-based. Without formal preparation in theories of education, novice clinical instructors may have significant deficits in their ability to effectively facilitate student learning in the clinical patient care setting and may rely on emulating teaching methods that were once used by a beloved educator or reliable colleague (Carrega \& Byrne, 2010; Ferguson \& Day, 2005).

Although clinical nursing educators are responsible for implementing teaching practices that promote learning in the patient-care area, limited research has been conducted to investigate and identify the in-the-moment teaching practices, knowledge, and grounding experiences that are specific clinical nursing educators who have transitioned to the role of a novice clinical nursing educator. In fact, teaching practices used in the clinical patient-care area are one of the 
least studied areas in nursing education (Hossein et al., 2010; Phillips \& Vinten, 2010). Patton (2007) stated:

There is a lack of nursing research particularly looking at the professional teaching knowledge of clinical nurse educators in relation to what they actually do in practice, the challenges they encounter, how they make sense of these challenges, and how they make decisions to respond in a manner consistent with their own practice (p. 489). Prior research studies examining teaching practices used by clinical nursing educators have not focused specifically on the in-the-moment teaching practices and underlying knowledge of clinical nursing educators who have transitioned to the role of the novice clinical nursing educator. This presents a gap in the nursing education literature. The findings of this study will inform field of nursing education of the teaching practices, knowledge, and grounding experiences particular to clinical nursing experts who transition to the role of a novice nursing educator teaching in the clinical patient-care area. 


\section{Chapter Three}

\section{Methodology}

\section{Introduction to the Research Methods}

The issue of schools of nursing hiring clinical experts, such as nurse practitioners or nurse practitioner students, with no educational preparation in teaching or pedagogy to fill the role of the clinical nursing educator was presented in chapter one. Chapter one also presented a historical perspective to explain how this phenomenon came to be and information as to why this phenomenon is predicted to continue into the foreseeable future. The review of the literature, presented in chapter two, examined research, professional standards, and provided a theoretical perspective from the disciplines of education and nursing education relating to the teaching practices and the lived experience of novice clinical nursing educators.

Chapter three begins with a review of the literature describing the methodological approaches and methods of data collection other investigators have used to study the teaching practices of clinical nursing educators. An overview of the research questions is also provided. Additionally, this chapter provides an overview of ethnography as a methodological approach to research as well as an argument for the appropriateness of this methodology as it applies to this research study. Additionally, a discussion is provided related to the theoretical perspective, rationale for selecting a focused ethnographic approach, and the research design for this investigation, including an overview the participants, recruitment efforts, the setting, data sources, and methods of data collection. Rationale for each of these design decisions is provided. Detailed information related to the framework used for data analysis and the process of data collection, ethical considerations, and researcher positionality statement conclude this chapter. 


\section{Review of the Methodological Literature}

A review of the literature demonstrates that the teaching practices of clinical nursing educators, how these teaching practices change in context, and the grounding experiences that shape and influence those teaching practices have been investigated through the use of various research methods and methodologies. These methods include: participant observation (Hsu, 2006; Ironside et. al., 2014; Mogan and Warbinek, 1994), semi-structured interviewing of novice clinical educators (Scanlan 2001; Twibell et al., 2005), comparative-descriptive research design (Phillips and Duke, 2001; Sellappah et al., 1998), and mixed-methods approach (Giddings et al., 2000). Although these investigations were conducted to explore the teaching practices used by clinical nursing educators, there have been no research investigations conducted to examine the particular clinical teaching practices used by clinical experts who transition into the role of the novice clinical educator. Additionally, there have been limited studies using focused ethnography as a research approach which uses the combined methods of field work, participant observation, ethnographic interviews, and document collection to investigate the teaching practices of clinical nursing educators. This gap in the research provides further credence for conducting this research study.

\section{Research Phenomenon}

The teaching practices and teacher knowledge that clinical nurse experts who have transitioned into the role of novice clinical nurse educators with no formal graduate preparation in education utilize when teaching in the clinical setting comprised the research phenomenon for this research study. Additionally, how these novice clinical nursing educators change or adapt their teaching practices when teaching undergraduate nursing students in the real-world clinical context was examined. Additionally, without formal education in teaching practices or theory, 
the unique grounding experiences of the teaching practices used by these clinical experts was investigated. Finally, areas of teacher knowledge and teaching practices that may be further developed through continued professional development were identified. These areas may be used to aid future clinical experts in the transition to novice clinical nursing educator.

\section{Research Questions}

Four overarching research questions that guided this study:

1. What teaching practices and teacher knowledge do novice clinical nursing educators demonstrate or draw from when teaching undergraduate nursing students in the clinical patient-care area?

2. In what ways do novice clinical nursing educators change or adapt their teaching behaviors as a response to the context of the teaching/learning environment?

3. What grounding educational or professional experiences do novice clinical nursing educators refer to or draw upon when working in the role of the clinical nursing educator?

4. What are areas of teacher knowledge and/or teaching practices, utilized by novice clinical nursing educators, which preclude the facilitation of student learning and/or critical thinking?

As these questions involve the investigation of a particular culture (i.e. clinical nursing experts who have transitioned into the role of a novice clinical nursing educator) focused ethnography was chosen as an appropriate research methodology to begin to seek answers to the research questions. Research questions in focused ethnographies often focus on the "what" and are used to describe experiences, beliefs, values, languages and/or behaviors experienced within 
cultural contexts or groups (Higginbottom, Pillay, \& Boadu, 2013). The following sections will provide rationale for the choice of this research methodology.

\section{Ethnography as a Research Methodology}

Ethnography has been defined as "both a product of research and a research process" (LeCompte \& Schensul 2010, p. 5). As such, ethnography is a multifaceted and complex research methodology with multiple definitions, purposes, and theoretical perspectives. Numerous authors have defined the work, purpose, or product of ethnographic research. Van Maanen (2011) defines ethnography as a "written representation of culture or selected aspects of a culture" (p. 1). Creswell (2018) articulates ethnography is a way of designing a qualitative research study in order to interpret and describe a learned pattern of beliefs, values, languages, or behaviors of a culture. LeCompt and Schensul (2010) argue that "ethnography is a systematic approach to learning about the social and cultural life of communities and other settings" (p. 1). Additionally, as a research methodology, ethnography allows the researcher to discover what people are actually doing before trying to interpret their actions (LeCompte \& Schensul, 2010). Spradley (1979) described ethnography as "the work of defining a culture" (p. 3). Based on these author's statements, it is clearly evident that at the heart of ethnography is the study of a culture.

Like ethnography, culture has been defined in many different ways. Roper and Shapira (2000) believe that there are two main conceptualizations of culture: behavioral/materialistic and cognitive. The behaviorist perspective includes observing a group's patterns of behavior and customs, whereas a cognitive perspective would include the ideas, knowledge, and beliefs that are held by a particular group of people (Roper and Shapira, 2000). Fetterman (1989) supports the cognitive view of culture when stating "culture comprises the ideas, beliefs, and knowledge that characterize a particular group of people” (p. 27). McCurdy, Spradley, and Shandy (2008) 
describe culture as "knowledge that is learned and shared and that people use to generate behavior and interpret experience" (p. 5). Spradley (1979) defines culture as "the acquired knowledge that people use to interpret experience and generate social behavior" (p. 5).

The novice clinical nursing educator participants in this research study are clinical experts who have no previous educational preparation in teaching. In order to fulfill the role of a clinical nursing educator they have transitioned from a clinical expert to a novice teacher. Therefore, the teaching practices and grounding experiences of these teaching practices are very unique to this particular group. Based on these definitions of culture, clinical experts who transition into the role of the novice clinical nursing educator would comprise a unique and individual culture.

Ethnography manifested as a viable research methodology for this research in order to gain a real-world perspective of the teaching practices, grounding experiences of these teaching practices, and an understanding of how these teaching practices change in relation to the context of the real-world clinical patient-care area. Using an ethnographic approach for this research study facilitated the exploration of several fundamental concepts of the culture of novice clinical nursing educators. First, the particular group of people under investigation were clinical experts who have transitioned into the role of a novice clinical nursing educator who have been assigned to teach undergraduate nursing students in the clinical patient-care area. By using ethnography as a method of qualitative inquiry, I was able to recognize and understand behavioral patterns of the novice clinical educators within contexts. These patterns included the teaching practices used by the educators, patterns associated with their interaction and communications with undergraduate nursing students (Robinson, 2013). I was also able to identify patterns or behaviors that the clinical nursing educators did not exhibit. 
Finally, by using an ethnographic approach, I was able to develop an understanding of the grounding experiences and knowledge that novice clinical nursing educators draw from when choosing to use particular teaching practices. To accomplish this, I interacted, observed, talked with, and questioned the novice educators that participated in this study in order to fully understand and identify what teaching practices they use and how these teaching practices changed in relation to the context, as well as why they use these particular teaching practices.

\section{Focused Ethnography}

There are numerous types of ethnographic studies as well as variant purposes for conducting an ethnographic research study. The type of ethnography that is conducted is dependent on the social or cultural group the ethnographer wishes to study (Morse, 1994). As such, I chose to use a focused ethnographic approach for this research study.

Focused ethnography, or microethnography, is a type of particularistic ethnography that focuses on a small group (perhaps less than 15 people), may focus on a distinct problem or distinguishing characteristic within a small group of people who have specific knowledge about an identified problem or issue, and offers a more narrow or specific focus for the ethnographic research (Higginbottom et al., 2013; Morse, 1994; Roper \& Shapira, 2000). Muecke (1994) suggests that the main features of focused ethnographies include: a problem-focused or context specific research approach based on a conceptual orientation of a single researcher, focus on a discrete or specific community, a limited number of participants who hold a specific knowledge, and episodic participant observation by the researcher rather than a "living among" approach that is used in macroethnographies. Focused ethnography is purposeful and utilizes the researchers' background knowledge to inform the research questions (Higginbottom et al., 2013). In a focused ethnographic study, the topic or culture to be examined is specific and may be identified 
by the researcher prior to beginning the study, with participants or informants chosen or selected based on their knowledge and experience (Higginbottom et al., 2013; Morse and Richards, 2002).

Roper and Shapira (2002) suggest two main purposes for using focused ethnography in nursing research. These purposes include using focused ethnography to understand meaning that members of a subculture assign to their experiences and to study nursing as a cultural phenomenon. According to Higginbottom (2013), focused ethnography "can be used to enhance and understand nursing practice by studying specific phenomena in distinct cultures and subcultures of clients or professionals" (p. 37).

A focused ethnographic approach was appropriate for this research study for the following reasons. The "what" research questions have been developed and guided the purposeful observations and questioning of the participants. The small group of participants in this research study were selected based on their specific and unique knowledge, experience, and characteristics. These unique characteristics sets them apart from the culture of other clinical nursing educators. Additionally, as this study was conducted by a single researcher, a conceptual approach based on the professional stance as a clinical nursing educator and knowledge of the literature gained through scholarly inquiry prior to data collection was used. Finally, the results of this study may be used to enhance the knowledge of the nursing education community as it relates to the teaching practices used by novice clinical nursing educators (Higginbottom, 2013). This newly acquired knowledge may be used to inform future professional development activities and programs for novice clinical nursing educators. In conclusion, the methodology of focused ethnography is appropriate for this proposed research study, as the research approach 
will be inclusive of a small number of participants with unique characteristics in a particular setting (the clinical patient-care setting) via an episodic-like manner.

\section{Epistemological and Theoretical Perspective}

Researchers and scholars offer different viewpoints and arguments related to defining or describing the epistemological and /or theoretical perspectives grounding ethnography. The theoretical perspective of ethnography can differ based on the aim of the researcher or the research study (Creswell, 2018). For example, theoretical perspectives in ethnography can include feminism, anthropology, Marxism, ethnomethodology, critical theory, structural functionalism, or symbolic interactionism (Creswell, 2018). For this proposed research study, the epistemological perspective of constructionism was used as is applies to the theoretical perspective of symbolic interactionism. The following section will provide rationale for this perspective.

Crotty (2015) suggests three distinct epistemologies in social research: objectivism, subjectivism, and constructionism. According to Crotty (2015), the epistemology of constructionism is embodied in the theoretical perspective of symbolic interactionism or interpretivism, or "an approach to understanding society and explaining the human world" (p. 3). The theoretical approach of constructionism implies that meaning is not discovered, but rather it is constructed (Crotty, 2015). Crotty (2015) suggests that ethnography is, therefore, informed by the theoretical perspective of symbolic interactionism because ethnographic inquiry "seeks to uncover meanings and perceptions on the part of the people participating in research, viewing these understandings against the backdrop of the people's overall worldview or culture" (p. 7). Similarly, Creswell (2018) argues that the concept of interpretivism may be used to describe the concept of social constructivism. In social constructivism, people or groups "seek understanding 
of the world in which they live and work" (Creswell, 2018, p. 24). In using this theoretical lens, researcher seeks to gain the participants' view of the situation in which they are placed and how they construct meaning through interaction within that situation or environment. The researcher, therefore, influenced by their own background or life experiences, also interprets meaning constructed from what he or she sees, hears, and experiences (Creswell, 2018).

For this research study, the lenses of constructionism, symbolic interactionism, and social constructivism were drawn upon. From an epistemological perspective, as guided by the theories of constructionism and symbolic interactionism, the purpose of this proposed research study was to seek and construct new meaning by gaining an understanding of the teaching practices used by novice clinical nursing educators and the grounding experiences of these teaching practices. The novice nursing educators were observed and interviewed as they teach undergraduate nursing students in the "real-world" patient-care area, within their own environment or situation. Based on the theoretical perspective of social constructivism, I also reflected my role as an interpreter in the process of understanding and reflecting upon my personal and professional background, experiences, and observations while constructing an ethnographic record of the teaching practices used by novice clinical nursing educators.

\section{Research Study Design}

A focused ethnographic research approach guided the research design in the following ways. The researchers' background as a seasoned clinical nursing educator informed the four what" research question (Higginbottom et al., 2013). The distinct clinical teaching practices, knowledge, and grounding experiences of clinical nursing experts who transition to the role of the novice clinical nursing educators comprised the specific phenomenon under investigation. A small number of participants who met specific research criteria were recruited for this study 
(Higginbottom et al., 2013). Saturation of data dictated the sample size (Higginbottom et al., 2013). Data was primary collected through episodic participant observation, however these methods also included ethnographic interviewing. During data collection, I was immersed in the culture of the novice clinical nursing educator in order to gain the perspective of the teaching practices and the participants' grounding influences. Observations of the clinical teaching practices took place in an episodic-like manner. Each participant was observed during three clinical days during the course of one semester, rather than being imbedded in practice with them over a long period of time. Additionally, only specific behaviors that related to the research questions were recorded and analyzed. I did not record behaviors of the novice nursing educator that did not correlate to the research questions. These behaviors included: the methods the novice nursing educators utilize to provide formative and summative evaluations of nursing students in the clinical patient-care area, grading practices, processes of selecting particular patients to be assigned to nursing students, and the student nurse perspective, to name a few.

During the analysis process, data from each participant was analyzed separately, and then again as a part of the participant group as a whole. This was a cyclic process which included my self-reflection as recorded in a research journal (Higginbottom et al., 2013). Documents, including the participant's curriculum vitas were used along with the course syllabi for their assigned courses as part of the analysis process (Higginbottom et al., 2013). The section below further describes the design of the research study, participant section, and data collection sources as they relate to the stated research questions. This section is followed by a detailed description of the data analysis. 


\section{Participants}

Four novice clinical nursing educators participated in the study. Criterion-based sampling was used as a recruiting method to select participants who met specific inclusion criteria of the study (LeCompte \&Schensul, 2010). Convenience sampling, or selecting participants who are immediately or geographically accessible, was used as an additional recruitment procedure for this study (Miles, Huberman, \& Saldana, 2014). As I am currently employed at a large university in the Mid-Atlantic region, all four participants were known to me prior to consenting to the study.

Participants of all ages and cultural backgrounds who meet the criteria for the study were considered for this study. Participants were required to meet several pre-determined inclusion criteria in order qualify as a participant in this research study. Inclusion criteria included the following:

- Enrolled or completed undergraduate and graduate education in nursing and working towards or hold a master's of science in nursing (MSN) degree.

- The graduate or undergraduate education could not have included courses in nursing education, education theory, or clinical teaching strategies.

- A minimum of four years of full-time experience as a registered nurse (RN)

- Three years or less of experience teaching undergraduate nursing students in the clinical patient-care area.

- Assigned to teach undergraduate nursing students in a medical-surgical patient-care unit. Exclusion criteria for this study included the following:

- Enrolled or completed a Master's of Science in nursing (MSN) in a non-clinical area (i.e. leadership, management, education, or other non-clinical graduate nursing degrees). 
- Obtained a doctoral degree ( $\mathrm{PhD}$ or $\mathrm{EdD})$ in education.

- Instructed students in a specialty area including pediatrics, psych/mental health, intensive care, obstetrics.

- More than three years of teaching experience.

- Less than four years of experience as a registered nurse.

\section{Number of Participants}

As this research study utilized a focused ethnographic approach, which includes a small sample of participants, prior to data collection, it was decided that either four or five participants would be recruited for the investigation (Morse, 1994; Roper \& Shapira, 2000). Upon recruiting and observing each participant, a detailed ethnographic record was created. After performing an in-depth analysis of the ethnographic record and semi-structured interviews of four participants, I concluded that data saturation had been reached. Therefore, recruitment efforts concluded. Four novice clinical nursing educators comprised the participant group for this study.

\section{Introduction to the Participants}

Based on a review of the participants' curriculum vitae, the following section provides a brief introduction to the participants, the types of students they were assigned to teach, and the clinical patient-care settings where data collection occurred. All participants were considered novice clinical nursing educators and provided direct supervision of undergraduate nursing students in a clinical patient care area. However, their backgrounds, professional experienced, education, and the clinical units in which they were assigned to teach varied significantly.

All four participants held undergraduate degrees in nursing. The participants professional experience as a Registered Nurse ranged from 5 to 25 years and included caring for patients in the following clinical areas: the emergency department, cardiac cath lab, hemodialysis center, 
trauma, intensive care, a medical clinic setting, and at a correctional facility. Three of the participants held a certification as a Nurse Practitioner. One participant was enrolled part-time in a Family Nurse Practitioner graduate program. The participants held between 1 to 19 years of experience as Certified Nurse Practitioners in the following clinical areas: gynecology and women's health, breast care, palliative care, and internal medicine.

Although all participants were considered novice clinical nursing educators, with less than three years of teaching experience, the years of teaching experience varied among participants. During the time they were observed, two participants were completing their third year of teaching in an undergraduate program, and two were completing their first year of teaching. All participants had at least one semester of teaching experience in the clinical setting prior to being enrolled in the study.

The type of nursing program, the academic level of the students, the academic semester the observations occurred, and the clinical areas in which participants were observed also varied. Due to the variation in the number of hours in the clinical day, the total number of observational hours also differed among participants. The participants were responsible for the clinical instruction of nursing students enrolled in either a traditional four-year or an accelerated BA/BSBSN nursing program at a large Mid-Atlantic University. All participants were observed teaching in medical-surgical clinical areas at a large Level 1 trauma center associated with the university. A description of the level of student, the clinical patient-care area, the total number of hours, and the time period of observation is provided below in Table 3.1. All participants have been assigned gender neutral pronouns pseudonyms as well as gender neutral pronouns in order to protect their identities. 
Table 3.1

Participant Information

\begin{tabular}{|c|c|c|c|c|c|}
\hline $\begin{array}{l}\text { Participant } \\
\text { Pseudonym }\end{array}$ & Semester & Clinical hours & $\begin{array}{l}\text { Total hours } \\
\text { of } \\
\text { observation }\end{array}$ & $\begin{array}{l}\text { Academic } \\
\text { Level of } \\
\text { Student }\end{array}$ & $\begin{array}{l}\text { Type of } \\
\text { Clinical } \\
\text { Area }\end{array}$ \\
\hline Alex & $\begin{array}{l}\text { Spring } \\
2019\end{array}$ & $\begin{array}{l}\text { 7:30am- } \\
\text { 12:30pm x } 3 \\
\text { observational } \\
\text { days }\end{array}$ & 15 & $\begin{array}{l}\text { Second- } \\
\text { semester } \\
\text { BA/BS-BSN }\end{array}$ & $\begin{array}{l}\text { Medical- } \\
\text { Surgical/ } \\
\text { orthopedic } \\
\text { unit. }\end{array}$ \\
\hline Bailey & $\begin{array}{l}\text { Summer } \\
2019\end{array}$ & $\begin{array}{l}\text { 7:30am-3:30pm } \\
\text { x } 3 \text { days }\end{array}$ & 24 & $\begin{array}{l}\text { First-semester } \\
\text { BA/BS-BSN }\end{array}$ & $\begin{array}{l}\text { Medical- } \\
\text { surgical, } \\
\text { step-down } \\
\text { unit }\end{array}$ \\
\hline Chris & Fall 2019 & $\begin{array}{l}\text { 7:30am- } \\
12: 30 \mathrm{pm} \times 3 \\
\text { days }\end{array}$ & 15 & $\begin{array}{l}\text { Second- } \\
\text { semester } \\
\text { traditional }\end{array}$ & $\begin{array}{l}\text { Medical- } \\
\text { Surgical/ } \\
\text { orthopedic } \\
\text { unit. }\end{array}$ \\
\hline Dana & Fall 2019 & $\begin{array}{l}\text { 7:30am- } \\
\text { 12:30pm x } 3 \\
\text { days }\end{array}$ & 15 & $\begin{array}{l}\text { First-semester } \\
\text { traditional }\end{array}$ & $\begin{array}{l}\text { Medical- } \\
\text { surgical, } \\
\text { step-down } \\
\text { unit }\end{array}$ \\
\hline Total hours & & & 69 & & \\
\hline
\end{tabular}

Prior to beginning recruitment for participants, all permissions for conducting this research study were completed. The Institutional Review Board (IRB) at West Virginia University approved the study on February 19, 2019.

\section{Data Collection Methods}

In order to gain a robust account of the teaching practices and grounding experiences of clinical nursing experts who transition into the role of the novice clinical nursing educator, several methods of data collection were employed for this investigation. These methods included field work and the writing of field notes and jottings, in-the-moment ethnographic interviewing, journaling, document collection, and semi-structured interviewing. The following sections 
provide a description of the observational setting, time frame for data collection, and how each method of data collection was utilized.

\section{Field Work and Observational Setting}

Spradley (1980) suggests that field work is performed not to study a particular culture or population, but to learn from a particular culture or population. Field work involves the investigator conducting research in situ, in the context or setting where the participants works or lives (Creswell, 2018; Leininger, 1984). Field work was used to answer each of the four research questions.

During this investigation, the novice clinical educator participants were observed in three different settings where the work of teaching took place: the pre-conference, the clinical patientcare area, and post-conference settings. Pre-conference was conducted by the clinical nursing educator for approximately one hour prior to the clinical day in a room or area that was designated by the school of nursing and in close proximity to the clinical unit. During the preconference sessions, the novice clinical educator would greet the students, make causal conversion, answer questions, provide direction for the clinical day, and listen to the student reports on their assigned patients.

The location and type of clinical patient-care setting was, again, assigned by the school of nursing. The four clinical nursing educators were assigned to teach clinical in a large acute-care hospital in the mid-Atlantic region of the United States. For this research study, observations took place in either an orthopedic medical-surgical unit and/or a medical-surgical step-down unit.

Post-conference sessions were conducted after completion of the clinical day. Some post conference sessions were conducted in a designated room in close proximity to the clinical area. 
Other times, post conference sessions were conducted in an area chosen by the clinical educator on or adjacent to the clinical unit. Post-conference sessions varied in time, depending on the clinical educator, from 5 minutes to approximately 30 minutes.

\section{Time Frame}

In order to facilitate an intimate in-depth study of teaching practices used by novice clinical nursing educators, each novice clinical nursing educator was observed for three clinical days during either the spring, summer, or fall semesters of 2019 (Merriam \& Tisdell, 2015). On average, one day of observation during the fall and spring semesters comprised around 5 hours. In the summer semester, an observation day averaged around 8 hours. Three days proved to be an appropriate number of days spent with each participant in order to answer the research questions. During the first day of data collection for each participant, a detailed ethnographic record was created in order to record what the clinical instructor was saying, doing, where the clinical instructor was going, and their interactions with the student. Upon conclusion of the first day of data collection, I analyzed the ethnographic record to identify behaviors that specifically related to the research questions. This allowed me to focus on particular teaching practices used by the clinical nursing educator and to gain an understanding of areas to focus observations and ethnographic questions during sequent observations. During the second day of observation, I focused on particular teaching practices, noting patterns, and similarities in behaviors displayed by the clinical nursing educator that were similar to the first day of observations. Upon conclusion of the third day of data collection, I was able to gain a clear understanding of the behaviors and teaching practices of the individual participants. Additionally, as observations were conducted with each participant, I gained an understanding of the behaviors and teaching practices of the participant group as a whole. 


\section{Participant Observation}

Participant observation is a "method in which a researcher takes part in the daily lives, rituals, interactions and events of a group of people as one of the means of learning the explicit and tactile aspects of their routines and their culture" (Dewalt \& Dewalt, 2010, p. 12). Taking the etic, or outsider stance, will allow the researcher to make comparisons and understand differences in behavior (Morse and Richards, 2002). In the participant-as-observer role, the researcher is able to validate observations with the participants through observing, interviewing, and recording field notes, and gain key information about what is like to be or work as a member of that culture or participant group (Roper and Shapira, 2002). The goal of observer-asparticipant role is to collect specific data. Contact with the participants that may be more formal and less intimate than in the participant-as-observer role, as the researcher takes on more of an observer role and participates less in the daily lives of the participant group (Roper and Shapira, 2002). Roper and Shapira (2002) suggest that moving between the participant-as-observer role and the observer-as-participant role may offer the best opportunities for the researcher to gain an understanding of the meaning of the group. As suggested by Roper and Shapira (2002) I moved between the roles of participant-as-observer role and the observer-as-participant for this investigation. Participant observation was used to answer each of the four research questions.

During the pre-conference sessions, I observed and made notations from either the back or the side of the room, with both the instructor and the students in view. In this way, the interactions between the clinical nursing educator and the nursing students were easily observable. These interactions were recorded via field notes and jottings. According to Emerson, Fritz, and Shaw (1995) field notes "are accounts describing experiences and observations the researcher has made while participating in an intense and involved manner" (p. 5). Emerson et 
al., (1995) state that jottings could be a few words up to several pages of description, depending on the setting and what the researcher is observing. Conversations between the instructor and individual students or groups of students were recorded as close to verbatim as possible.

As pre-conference concluded, I accompanied the clinical nursing educator and the nursing students to the clinical unit. Once on the clinical unit, the clinical nursing educator was observed as they went about their day. This included observing the clinical nursing educator as the provided clinical direction to the nursing students, prepared medications for administration, assisted students with computer charting, worked with the staff on the clinical unit to coordinate patient care, retrieved medications and supplies, and answered student questions. Observations took place in the hallways of the clinical unit, medication room, and clean utility rooms. At times, I was able to stand outside of the patients' room, out of sight of the patient, and observe the interaction between the clinical nursing educator and the student. During or immediately after observations between the clinical nursing educator and the student(s), I would hand-write indepth notes, including verbatim conversations between the clinical instructor and the student, in order to described actions, behaviors, teaching practices, responses, and questions asked by the clinical nursing educator. My personal thoughts, ideas, and reflections were also recorded during this time for future considerations.

Upon conclusion of the clinical day, the clinical instructor and students convened in the post-conference area. Again, observations and conversations were recorded as I sat either in the back or to the side of the room. Upon conclusion of post-conference, a few extra moments were spent with each instructor in order to ask additional questions and clarify observations. 


\section{Student and Patient Confidentiality}

During the course of data collection, at no times were students were identified by name or gender. In order to protect the identity of the students, students were addressed in field notes as "Student 1, Student 2", etc. Likewise, prior to data collection, the decision was made that student-clinical nursing educator interactions would not be observed in the patient's hospital room. Rather, the observations would be limited to patient care areas such as hallways and other patient-care areas. This decision was made for several reasons. First, it was of utmost importance to me to protect the privacy of the patient. As such, the patients were never identified by name, initials, or room number during data collection. Secondly, in no way did I want the patient or their family to feel their care was being compromised by either the student or the clinical nursing educator due to their participation in this study. This action was taken to assure that the student and clinical nursing educator were not distracted from providing care to the patient. Although this may have limited my ability to observe or capture the entirety of the interactions between the clinical instructor and the student, this was a compromise that I was willing to make in order to protect the patients' safety and confidentially.

\section{Ethnographic Interviewing}

Ethnographic interviewing, or interviewing done during participant observation in order to obtain a robust idea of the participant's insider or emic perspective was used as a data collection method in order to answer the research question: What grounding educational or professional experiences do novice clinical nursing educators refer to or draw upon (Fetterman, 1989; Spradley, 1979). According to Spradley (1979), ethnographic interviewing is more like having a conversation with a research participant, rather than a structured or semi-structured interview. Additionally, this type of interviewing may be done during participant observation in 
order to obtain a robust idea of the participant's insider or emic perspective (Fetterman, 1989; Spradley, 1979). Through the process of ethnographic interviewing and utilization of multiple forms of questions, I developed an understanding of the grounding personal or professional experiences influencing the teaching practices of the novice nursing educator participants. Additionally, some ethnographic interview questions were asked "on the spot" while observing the participants in order to clarify thoughts and/or ideas about what I was seeing or hearing or to ascertain additional information concerning the instructors teaching methods.

\section{Semi-Structured Interviewing}

Semi-structured interviews were used as an additional method of data collection for this research study in order to answer the research question: What grounding educational or professional experiences do novice clinical nursing educators refer to or draw upon when working in the role of the clinical nursing educator? Interviews took place in an area chosen by the participant including a private conference room and/or my personal office. The questions were asked of the participants in order to gain an understanding of the participants' perception of their role as a clinical nursing educator. Additionally questions sought to gain an understanding of the grounding personal or professional experiences they draw upon when working in the role of a clinical nursing educator. The four interview questions asked of each participant were:

- In the clinical patient care area, what role and/or responsibility do you feel you have as clinical nursing educator?

- Do you have a particular teaching style?

- Do you have a particular person you emulate when you are teaching? 
- Do you feel the clinical rationale you provide to the students is coming from your knowledge as a registered nurse, nurse practitioner (or NP student), or another source?

Interviews were recorded via an iPhone recording application. Transcripts of the interviews were transcribed verbatim.

\section{Document Collection}

Documents were used as an additional data source for this research study. Document analysis is utilized as a research method in order to elicit meaning, empirical knowledge, or a fuller understanding of the culture from texts, artifacts, or images (Bowen, 2009; Munhall, 2007). The course syllabi were collected and analyzed in order to gain an understanding of the clinical teaching objectives that the clinical nursing educator may be trying to meet. The course syllabus included the student learning objectives for the overall course as well as the clinical portion of the course. Also, the course syllabi were used in order to understand where on the curricular "horizon" of the overall nursing curriculum the clinical course was placed. This provided a source of reference in order to understand the previous concepts and skills introduced or covered in the current clinical course for which the clinical instructor was assigned.

Additionally, a curriculum vita (CV) of the participants were collected and analyzed in order to provide an understanding of the types and years of clinical experience, both as a registered nurse and as a clinical nurse practitioner, if applicable. The CV's were also used to verify the graduate preparation of the participants. Analysis of the participants' CV's provided an understanding of the participants' clinical expertise or strengths, as well as clinical areas where they lacked experience. 


\section{Reflective Journaling}

Qualitative research requires the researcher to self-reflect on the research process (Creswell, 2018; Lincoln, Lynham \& Guba, 2018). The researchers' background, work experience, culture, and prior experiences will affect his or her interpretation of the information and what the researcher hopes to gain from the study (Creswell, 2018). The researcher, in turn, must acknowledge his or her own influences on data collection and interpretation (Streubert \& Carpenter, 2011).

Therefore, in addition to utilizing several other research methods, reflective journaling or writing "in the margins" was used in order answer the research questions: What teaching practices do novice clinical nursing educators demonstrate when teaching undergraduate nursing students in the clinical patient-care area: In what ways do novice clinical nursing educators change or adapt their teaching behaviors as a response to the context of the teaching/learning environment? It was through the reflective journaling process that I was able to write and reflect upon the data collection process, connect participant observations to my personal and professional experiences, and pose additional questions to be explored in greater depth and breadth. This reflective journal was analyzed during the analysis phase of this research study. In conclusion, using criterion-based and convenience sampling, four participants consented to participate in this focused ethnographic research study. Multiple methods of data collection were used in this research study in order to answer the overarching research question. By using data collection methods such as field work, participant observation, and interviewing, a "real-world" description of the teaching practices used by novice clinical nursing educator's and the grounding experiences of these teaching practices were identified. 


\section{Data Analysis}

For this research study, a theoretical thematic qualitative data analysis approach was utilized in order to construct the meaning of the data as it relates to the research questions (Crotty, 2015). Analysis of data was conducted for each individual participant as well as the participant group as a whole to identify themes and patterns. The following section will describe the cyclic process of data collection and analysis, provide rationale for the use of a theoretical thematic approach to data analysis, introduce the theoretical framework of Pedagogical and Subject Matter Knowledge for Clinical Nursing Educators, as adapted from the Mathematical Knowledge for Teaching framework (Ball et al., 2008), and describe the process used for data analysis, methods used to maintain rigor of data analysis, ethical consideration, and my researcher positionality statement.

\section{Theoretical Thematic Data Analysis}

The process of Theoretical Thematic Analysis was used to analyze all forms of data. Thematic analysis is a qualitative descriptive analytic approach "for identifying and reporting patterns (themes) within data" (Braun \& Clark, 2006, p. 79). Thematic analysis fits within the constructionist theoretical perspective of this research study, as this type of data analysis is used to examine the realties, meanings, or events that are observed (Braun \& Clark, 2006). A review of the literature demonstrates that several focused ethnographic investigations in nursing have utilized thematic analysis during the data analysis phase (Dupuis-Blanchard, Neufeld, \& Strang, 2009; Ensign \& Bell, 2004; Kilian, Salmoni, Ward-Griffin, \& Kloseck, 2008; Pasco, Morse, \& Olson, 2004; Spiers \& Wood, 2010).

Thematic analysis can be either inductive, such as in grounded theory, or deductive, or based on a known theory (Braun \& Clark, 2006). Braun and Clark (2006) suggest that, although 
a theoretical thematic analytic approach may provide less of a description of the overall data, it will provide a more detailed description of some of the data. As this research study used a focused ethnographic methodological approach, based on the specific research questions, it was appropriate to use a theoretical thematic analytic approach in order to provide a detailed description of the specific teaching practices, context of these teaching practices, and grounding experiences of the teaching practices used by novice clinical nursing educators.

As discussed in chapter two, the discipline of nursing education lacks a framework that describes the content knowledge for teaching and/or the general pedagogical knowledge that is required of a clinical nursing educator. Therefore, the Mathematical Knowledge for Teaching (Ball et al., 2008) framework was adapted in order to understand the unique pedagogical knowledge and subject matter knowledge of a clinical nursing educator. The adapted framework, re-named the Pedagogical and Subject Matter Knowledge for Clinical Nursing Educators framework, was used as the thematic theoretical framework during data collection and analysis in order to identify, define, describe, and analyze the in-the-moment teaching practices and knowledge of novice nursing educators. Chapter four of this investigation includes an in-depth discussion of and research findings of this research study pertaining to each aspect of the adapted framework. Table 3.2 demonstrates the six facets of knowledge, as described in Ball et al.'s (2008) Mathematical Knowledge for Teaching model, how each facet was adapted to meet the constructs of clinical nursing education, and an example of how a clinical nursing educator would draw upon each facet of knowledge when teaching in the clinical patient-care area. 
Table 3.2

Pedagogical and Subject Matter Knowledge for Clinical Nursing Educators with Examples

\begin{tabular}{|c|c|c|}
\hline $\begin{array}{l}\text { Subject } \\
\text { Matter } \\
\text { Knowledge }\end{array}$ & $\begin{array}{l}\text { Application to Clinical Nursing } \\
\text { Education. }\end{array}$ & Example from Nursing Practice \\
\hline $\begin{array}{l}\text { Common } \\
\text { Content } \\
\text { Knowledge }\end{array}$ & $\begin{array}{l}\text { Common content knowledge (CCK) for } \\
\text { the clinical nursing educator includes } \\
\text { knowledge related to discipline of } \\
\text { nursing. This includes knowledge of } \\
\text { disease processes, nursing interventions, } \\
\text { and interpretation of laboratory values. } \\
\text { Additionally, this knowledge is inclusive } \\
\text { of the clinical nursing educator ability to } \\
\text { apply his or her experience as a clinical } \\
\text { nurse to the patient-care setting, } \\
\text { including utilization of clinical judgment } \\
\text { and critical thinking skills as they relate } \\
\text { to the acute care clinical setting. This } \\
\text { knowledge would have been acquired } \\
\text { during clinical nursing educators' } \\
\text { undergraduate or graduate nursing } \\
\text { education and/or through clinical } \\
\text { practice. }\end{array}$ & $\begin{array}{l}\text { The clinical nursing educator would use } \\
\text { CCK when instructing a student to not to } \\
\text { administer an anti-hypertensive medication } \\
\text { based on the patients' medical condition, } \\
\text { physical assessment, and blood pressure } \\
\text { reading. In this scenario, the clinical } \\
\text { nursing educator is drawing upon their } \\
\text { knowledge of actions of the medications, } \\
\text { their overall knowledge of the patient, and } \\
\text { their experience in administrating this } \\
\text { medication in clinical practice. }\end{array}$ \\
\hline $\begin{array}{l}\text { Specialized } \\
\text { Content } \\
\text { Knowledge }\end{array}$ & $\begin{array}{l}\text { Specialized Content Knowledge (SCK) is } \\
\text { inclusive of specialized knowledge that } \\
\text { the clinical nursing educator would need } \\
\text { to utilize when teaching nursing students } \\
\text { to care for patients in a clinical patient- } \\
\text { care area. This knowledge would be } \\
\text { specific to clinical unit routines, the role } \\
\text { of health care professionals, and the role } \\
\text { and responsibilities of the Registered } \\
\text { Nurse working in a clinical patient-care } \\
\text { area, and everyday task of the registered } \\
\text { nurse including medication } \\
\text { administration and documentation in the } \\
\text { Electronic Medical Record (EMR). SCK } \\
\text { also includes knowledge of the nursing } \\
\text { process. The clinical nursing would have, } \\
\text { ideally, acquired specialized content } \\
\text { knowledge through clinical practice as a } \\
\text { Registered Nurse. }\end{array}$ & $\begin{array}{l}\text { SPK would be used by the clinical nursing } \\
\text { educator when providing directions to a } \\
\text { student on the correct method to document } \\
\text { that an hypertensive medication was } \\
\text { withheld and not administered to the } \\
\text { patient based on the patients' medical } \\
\text { condition, physical assessment, and blood } \\
\text { pressure reading. }\end{array}$ \\
\hline
\end{tabular}


Horizon Horizon Content knowledge (HCK)

Content

Knowledge

involves the clinical nursing educators'

knowledge of what content is important

to each in the clinical patient-care area. It

also includes the clinical nursing

educators' knowledge of how the content

taught in the clinical patient-area

connects to the students' prior knowledge

and future learning. Examples of this

would include the clinical nursing educators' ability to engage the students'

prior knowledge from general education courses, such as the sciences and of preceding nursing courses. HCK would include the clinical nursing educators' ability to relay information appropriate to the level of the learner. HCK would enable to the clinical educator to ask appropriate questions based on students' prior learning. Finally, HCK would enable the clinical nursing educator to scaffold content in the clinical setting based on their knowledge of the broader disciplinary learning needs.

\begin{tabular}{|c|c|}
\hline $\begin{array}{l}\text { Pedagogical } \\
\text { Content } \\
\text { Knowledge }\end{array}$ & Application to Mathematics \\
\hline $\begin{array}{l}\text { Knowledge } \\
\text { of Content } \\
\text { and Students }\end{array}$ & $\begin{array}{l}\text { Knowledge of content and students } \\
\text { (KCS) includes the clinical nursing } \\
\text { educator's understanding how nursing } \\
\text { students learn or come to understand } \\
\text { concepts in the clinical patient-care area. } \\
\text { This may include the selection of specific } \\
\text { examples used in the patient care area in } \\
\text { order to clarify or explain a concept. It } \\
\text { also includes the clinical nursing } \\
\text { educators' ability to anticipate what a } \\
\text { nursing student may understand as well } \\
\text { as common misconceptions. KCS may be } \\
\text { used to help a student to understand how } \\
\text { terms may be used in different ways on } \\
\text { the clinical unit. }\end{array}$ \\
\hline $\begin{array}{l}\text { Knowledge } \\
\text { of Content } \\
\text { and } \\
\text { Curriculum }\end{array}$ & $\begin{array}{l}\text { Knowledge of Content and Curriculum } \\
\text { (KCC) includes the clinical nursing } \\
\text { educators' knowledge of course and } \\
\text { program objectives, clinical objectives, }\end{array}$ \\
\hline
\end{tabular}

HCK would be used to question the student related to their knowledge of the body's regulation of blood pressure. The clinical nursing educator would know that this knowledge would be derived from the students' pathophysiology course they took as part of their general science courses. HCK would enable the clinical nursing educator to provide information at the level that the particular learner would be able to comprehend. Also, in order to scaffold the students learning, HCK would also be used to guide the clinical nursing educator in questioning the student as to what comorbidities may be complicated by the disease process of hypertension.

Application to Clinical Nursing Education
Examples of this would include the clinical nursing educators' choice of using an analogy or real-world example to help facilitate the students' understanding of a difficult concept or skill. For example, the clinical nursing instructor may use the analogy of a water pump and water pipes to explain the concept of peripheral vasodilation and peripheral vasoconstriction that may affect a patients' blood pressure.

An example of this would be the clinical nursing educators' ability to decide which nursing interventions are appropriate for a sophomore level nursing student working 
and clinical assignment objectives. Additionally, this would include the clinical nursing educators' knowledge of appropriate skills and nursing interventions the nursing student may perform or not perform independently or with supervision based on their level in the nursing program.

Knowledge of Content and Teaching

Knowledge of Content and Teaching (KCT) includes the clinical nursing educators' knowledge of specific teaching practices that facilitate learning and critical thinking in the clinical patient care area. These include the teaching practices of questioning, promoting discussion, active engagement, and role modeling. KCT also includes the clinical nursing educators' ability to choose the correct teaching method for in-themoment instruction of students. KCT for clinical nursing educators also incorporates constructs from Shulman's (1986) general pedagogical knowledge including classroom management and organization and classroom communication and discourse GessNewsome \& Lederman, 2001). in the clinical patient care area. For example, based on the clinical objectives of the course, the clinical nursing educator would know that this level of nursing student would need to be directed and supervised to utilize a manual blood pressure cuff, rather than using an electric or automatic cuff, to assess a patients' blood pressure.

An example of this may include the clinical nursing educator choosing to utilize the teaching practice of discussion during the post-conference session to engage students and facilitate critical thinking. For example, the clinical nursing educator may choose to lead a discussion in the postconference session related to the various assessments and treatments for hypertension that the students identified during their clinical day. The clinical nursing educator may also choose to utilize scenarios or brief case studies in order to facilitate the students' discrimination of normal and abnormal assessment findings, and appropriate interventions and/or medications that may be administered based on assessment findings.

\section{Process of Data Analysis}

Roper and Shapira (2000) suggest that data analysis actually begins while data is being collected as the researcher reflexively discovers themes and patterns and allows those discoveries to influence their questions and observations. The first step of this process involved reading and re-reading transcripts and field notes and jotting down thoughts and ideas based on each individual participant, and as the participant group as a whole. Upon completion of each participant observation in the clinical patient-care area, the hand-written field notes and jottings recorded during participant observations were typed and organized. This process included 
transcribing notes of conversations between the student and the clinical nursing educator. It also included notes and recordings of conversations and interviews with the clinical nursing educator.

As suggested by Roper and Shapira (2000) and Spradley (1979), a cyclic process of observing, collecting, transcribing, and analyzing the data occurred after each observation session with a participant. In conducting a cyclic process of observing, interviewing, and analyzing, I was able to reflect upon the meaning of the collected data in order to identify further questions to pose in subsequent interviews (Spradley, 1979). Analysis of the data was completed for each participant as an individual, as well as the participant group as a whole. Additionally, new thoughts, ideas, and themes were recorded and reflected on these via the reflective journal. Through the process of observation, writing, and reflecting, additional ethnographic interview questions were drafted and asked of the participant during subsequent observation days. It was through this cyclic process that I began to recognize and understand patterns of repeating behaviors of each individual participant, as well among the participant group as a whole, emerged.

\section{Generating Codes}

Theoretical thematic data analysis is a multi-step process of data collection and analysis (Braun and Clark, 2006). Braun and Clark (2006) suggest that generating codes is first step in thematic analysis. Saldana (2016) suggests that descriptive coding is appropriate for ethnographic research studies that utilize interviews, field notes, documents, and journals. A descriptive code assigns a label to a passage of qualitative data in order to summarize or describe the data (Miles, Huberman \& Saldana, 2014). Descriptive codes were assigned to describe the clinical nursing educators' actions, reactions, and behaviors. The first descriptive codes to emerge during data analysis were "responding" and "questioning" to provide a basic description 
of the educators' behaviors. From there, additional codes were used to describe the types of responses made by the clinical nursing educator the students such as "providing clinical rationale" and "providing direction for care". Additionally, codes were developed to describe the types and purposes of questions asked by the clinical nursing educator such as "asking for additional clinical information" and "asking about completion of assignment." Other codes were developed to describe additional teacher actions including: "setting expectations,", "classroom management," and "teaching via personal stories." Finally, codes were developed to describe areas of future professional development. These codes included: "missed learning opportunities," "teaching as task," "asking questions above the level of the learner," and "failure to engage prior knowledge." All forms of data, field notes, documents, the reflective journal, and interview transcripts were coded via first cycle descriptive coding. Each code was color coded in order to visualize each code on the ethnographic record (see Appendix A).

\section{Generating Themes}

A second step in ethnographic data analysis, or second cycle pattern coding, is sorting the coded data or descriptive labels into a smaller number of sets in order to identify themes and patterns (Miles et al., 2014; Roper \& Shaperia, 2000; Saldana, 2016). A theme is an outcome of coding and analytic reflection and is used to represent a pattern or meaning within the data (Braun \& Clark, 2006; Saldana, 2016).). Saldana (2016) suggests that thematic coding is appropriate method for thematic analysis of interviews, field notes, and other participantgenerated documents and artifacts.

This second step in analysis involved identifying emerging themes, sorting the different codes into these themes, and organizing the data by thematic constructs (Miles et al., 2014; Saldana, 2016). Therefore, the codes created during the first-level analysis process, were 
analyzed in order to identify relationships, similarities, and differences, between and among the codes and identify emergent themes that correlated to the Pedagogical and Subject Matter Knowledge for Clinical Nursing Educators framework. Six themes of teacher knowledge were identified in this framework: knowledge of curriculum, knowledge of students, knowledge of teaching, common content knowledge, specialized content knowledge, and horizontal content knowledge. During this process, much consideration was given to how each theme each fit into or represented one of the six identified areas of teacher knowledge identified by the framework. In order to organize and visualize themes, both a narrative description and a thematic map was developed in order to organize and display discovered themes (Roper \& Shapira, 2000). This map was used to describe themes related to the participant group as a whole (see Appendix B).

\section{Developing an Understanding}

A third step in ethnographic data analysis is to explore the analyzed data to in order to construct a theoretical understanding of the phenomenon (Roper \& Shapira, 2000). Based on a detailed review of the literature, a prolonged period of observation, the development of codes and themes, I was able to develop an understanding of the knowledge, teaching practices, and grounding experiences used by novice clinical nursing educators when teaching undergraduate students in the clinical patient care area. Additionally, through careful analysis, insights emerged concerning the knowledge or teaching practices that were absent or poorly implemented. These findings represent teaching practices that may deter student learning, as well as opportunities for future professional development of novice clinical nursing educators. 


\section{Rigor in Data Analysis}

Several steps were taken to maintain rigor during the data analysis process. This included addressing the processes to maintain credibility, dependability, and confirmability (Lincoln \& Guba, 1986). The following sections address each of these processes.

\section{Credibility}

Credibility is an authentic portrait of the actions or behaviors of the participants under investigation (Miles et al., 2014). To enhance the trustworthiness or credibility of the research study, three different types of data (field notes, interview transcripts, and documents) were analyzed and coded in order to confirm findings (Miles et al., 2014). Prolonged or persistent engagement with the participants was an additional step in assuring credibility of the data (Lincoln \& Guba, 1986; Krefting, 1990). During the course of this investigation a total of 69 hours was devoted to observation of the participants with several additional hours devoted to interviewing them individually. According to Denzin (1989) a thick description of qualitative data may establish the sequence of the events or significance of the experience. This may also include the voices, actions, feelings and/or meaning of the interactions (Denzin, 1989). During the observational process, detailed notes of conversations, actions, and reactions of the participants were documented along with the location and contexts of the observations. Teaching practices used in the clinical setting were described in detail, and specific examples used. Additionally, ethnographic interviewing was used as a data collection method in order to gain "real-time" insight into the novice nursing educator's decision-making process and the reasoning behind using selected teaching practices in context. 


\section{Dependability}

Dependability was maintained through the research process by providing a dense description of the research methods and how each method was used to collect data (Krefting, 1990). A second method to ensure dependability was coding and re-coding the data after waiting for a period of time in order to compare the findings among and between each participant (Krefting, 1990). The data for each participant was coded as close to the observation period as possible. After coding of each additional participant's data, the previous participant's data was reviewed and recoded to assure consistency in coding. After all data was collected on all four participants, the data was reviewed and coded a final time in order compare all the participants' data and provide consistent coding.

\section{Confirmability}

Confirmability of the data was maintained through reflexive analysis of my bias and opinions that were captured via the reflective journal (Krefting, 1990). Shenton (2003) states "a key criterion for confirmability is the extent to which the researcher admits his or her own predispositions" (p. 72). Throughout the entire process of conducting this ethnographic research study, it was imperative that an in-depth and accurate account of what was actually being heard and seen was documented, not just my perception of what was being heard or seen (Roper \& Shapira, 2000). As such, direct quotations were used as much as possible during data collection. Additionally, throughout the entire research process I worked to continually write and reflect upon my biases and personal thoughts via the reflective journal. A final step that was taken in order to produce confirmability was keeping an audit trail, via the reflective journal, of the stepby-step research process. This included a description of participant recruitment, how the research 
data was collected and processed, and how the data related to the research questions (Shenton, 2004).

\section{Ethical Considerations}

As data was collected in a "real-world" learning environment in a healthcare institution, several steps were taken to maintain the confidentially and privacy of the participants as well as the students, staff members, patients, and families with whom they interacted. Prior to the study, the participants were made aware of the purpose of the study and its intended use (Strike et al., 2002). Participants were able to decide to participate in this study freely and without coercion and at any time. Participants had the right to withdraw from the study if they felt they no longer wanted to participate (Strike et al., 2002) Confidentiality was maintained in order to protect the names of the participants, their gender, the institution for which they teach, staff members on the clinical unit, the students assigned to their clinical group, and the patients for which the students are assigned to care (Strike et al., 2002). Pseudonyms were assigned to all students, staff, and instructors. Observations did not include interactions with the patients or the patient's family members in order to protect their Health Insurance Portability and Accountability Act (HIPAA)

privileges. Additionally, students were not identified by name, or class in which they are enrolled in order to protect their Federal Education Rights and Privacy Act (FERPA) privileges. An ethical approach was maintained by applying for and gaining Institutional Review Board (IRB) consent and having the participants in the study sign a consent form prior to beginning participant observation (Strike et al., 2002).

\section{Limitations}

The following limitations are true of this study:

1. The small sample size may not be representative of all novice clinical nursing educators. 
2. All participants will be selected from the same geographical region. This may limit transferability of findings to other geographical locations.

3. The novice clinical nursing educator may change methods of teaching due to the researcher functioning in the role of the observer.

4. Participants were assigned to teach in medical-surgical or fundamental nursing courses only. These findings may not be transferable to novice clinical nursing educators teaching in other clinical courses.

5. Researcher bias may influence analysis of results. Because the researcher has been teaching as a clinical instructor for over 18 years, bias to the role of the clinical educator may play a part in the collection, analysis, and interpretation of data.

\section{Researcher Positionality}

LeCompte and Schensul (2010) argue that it is important for researches to identify positions of power that may be assumed by the researcher prior to conducting the research study. Therefore, it is important to include a bit of my own story as part of this ethnography. I am academic nursing educator with a master's degree in nursing education. I have over 18 years of experience teaching undergraduate nursing students in the classroom and the clinical patient care area. I have worked as a registered nurse for over twenty-three years. I currently hold a nontenured position at a large university in the Mid-Atlantic region at the rank of Clinical Education Associate Professor. I have been enrolled in a Doctorate of Education program with a focus on curriculum and instruction as part of this dissertation process. I have also served as preceptor and mentor to new clinical nursing educators who enter into the clinical teaching profession. In addition, I hold certification through the National League of Nursing (NLN) as a Certified Nursing Educator (CNE). Through these professional and educational experiences, I have had 
the opportunity to become very familiar with nursing curriculum, pedagogy, learning needs of the undergraduate nursing student, as well as clinical teaching practices that facilitate student learning. This education and experience also has afforded me the ability to recognize teaching practices that preclude the facilitation of student learning. Although these experiences greatly influence what I know and have come to learn about clinical education and working as a clinical educator, they also require me to examine and profess my role and potential bias as an "expert" when observing "novice" clinical nursing educators teach in the clinical patient-care setting.

As I entered into and frequently throughout data collection, I would reflect on Benner's (2001) Novice to Expert theory in order to understand both the position of the novice clinical nursing educator and my position as an expert clinical educator. According to Benner's (2001) Novice-to Expert framework, when making clinical decisions, experts draw upon their years of nursing experience, intuition, background, and a deep understanding of the situation. Their performance is now flexible, fluid, and highly proficient. They recognize a problem and simultaneously know how to solve it (Altmann, 2007). My professional experience as a registered nurse, a clinical nursing educator, and a doctoral candidate have clearly influenced my understanding of the role and responsibilities of clinical nursing educators. During observational sessions with the participants, I would often ask "what would I do in this situation" or "how would I handle this situation?" I think these thoughts are natural, considering on days that I was not collecting data in the role of the researcher, I was working in the role of the clinical nursing educator and teaching students in the clinical patient care area, just as the participants were doing. At first, I worked to close off these thoughts in order to be as objective as possible in thinking only about the participants' actions and behaviors, and not my own. However, as I progressed through the data collection and analysis process, I realized that, as an expert, my 
thoughts, ideas, and perceptions were not only valid but important to this study, and that I must use these personal experiences in order to create this ethnographic record. It was through these reflections on my own practice that allowed me to identify and understand the teaching practices used by the participants that prohibit learning and/or may constitute areas of continued improvement for novice clinical educators.

The goal of this study was to identify knowledge, teaching practices, and experiences that clinical nursing experts who transition to the role of novice clinical nursing educator's use when teaching in the clinical patient care area. A second goal was to understand and define knowledge and teaching practices that were either unfamiliar to the participants, under-utilized by the participants, or actually deterred student learning in order to inform the development of continuing professional education. I have come to understand that my personal knowledge, education, and experiences enable me to notice, make judgments, and confidently identify and describe teaching practices, knowledge, and grounding experiences of the participants. It is my hope that the findings of this study may be used in order to provide empirical evidence to guide the design and implementation of future professional development activities and programs for novice clinical nursing educators.

\section{Conclusion}

The purpose of this chapter was to provide a rich description of the research design, the ethnographic methodology used, and the specific methods of data collection and analysis. The data used in this study was described in detail and included participant observation, ethnographic interviewing, and document collection. Analysis of the data focused the knowledge, teaching practices, and underling influences of those teaching practices used by novice clinical nursing educators when teaching students in the clinical setting. This chapter also provided information 
pertaining to maintaining student and patient confidentiality, ethical considerations, limitations, concepts related to rigor in data collection, and a statement of researcher positionality. 


\section{Chapter Four}

\section{Findings}

\section{Introduction to the Research Findings}

The purpose of this chapter is to provide a detailed ethnographic account of the findings of this investigation related to the teaching practices, knowledge, and grounding experiences of the four participants. The participants were clinical nursing experts who transitioned into the role of novice clinical nursing educator. To provide this detailed account, chapter four is divided into three distinct sections. This chapter begins by introducing each of the participants and providing a summary of their professional experiences. Additional findings pertaining to the particular professional or personal grounding experiences that have influenced, shaped, or defined the individual teaching practices of each participant are provided. This section also provides the reader with insight as to why the participants may have used or not used a particular teaching practice or practices when teaching undergraduate nursing students in the clinical setting. The second section of this chapter provides a detailed account of the teaching practices used by the participants when teaching in the clinical patient care area. Additionally, a description of when, where, and/or in what context, the teaching practices were used is provided. The final section of this chapter is devoted to identifying and describing research findings that pertain to teaching practices that were either under-utilized by the participants and/or prohibited student learning. This section may be used to inform future professional development in order to facilitate clinical nursing expert's transition into the role of a novice clinical nursing expert. As discussed in chapter's two and three, the Pedagogical and Subject Matter Knowledge for Clinical Nursing Educators framework, adapted from the theoretical framework, Mathematical Knowledge for Teaching (Ball et al., 2008), is used as the guiding theoretical lens though which the findings are viewed. 


\section{Section One: Introduction to the Participants and Grounding Experiences}

As described in chapter three, for this focused ethnographic study, four novice clinical educator participants were recruited, observed, and interviewed during the data collection process. In order to participate in the research study, the participants met the following inclusion criteria: (a) enrolled or completed undergraduate and graduate education in nursing and working towards or hold a master's of science in nursing (MSN) degree, (b) their graduate or undergraduate education did not include courses in nursing education, education theory, or clinical teaching strategies, (c) four years of full-time experience as a registered nurse (RN), (d) three years or less of experience teaching undergraduate nursing students in the clinical patientcare area, and (e) assigned to teach undergraduate nursing students in a medical-surgical patientcare unit. Based on the inclusion criteria, the participants' years of experience as either a registered nurse or a nurse practitioner would qualify them to be considered an expert nurse. Additionally, as none of the participants had more than three years of teaching experience, for the purpose of this study, the participants were all considered to be novice clinical educators.

One of the aims of this research study was to investigate what grounding experiences novice clinical nursing educators with no formal graduate education in educational theories, pedagogy, or teaching practices draw upon when teaching or making teaching decisions in the clinical patient-care area. To accomplish this, data including semi-structured interviews, ethnographic interviews, and observations were analyzed to uncover and identify the participants' grounding experiences and the influence these grounding experiences had on their teaching. The following sections introduce each of the participants (Alex, Bailey, Chris, and Dana), as well as discusses the findings related to this research aim. These finding include the following information about each participant: the participant's unique professional nursing 
experience, their perception of the role of the clinical nursing educator, the grounding experiences that have influenced their perception of the role and/or their clinical teaching practices, and their perception of their teaching style.

\section{Alex's Grounding Experiences}

Alex has over twenty years of experience as an RN and four years of experience as a Family Nurse Practitioner. Alex has worked in a variety of clinical settings including a correctional facility and in medical clinics both in the United States and abroad. Alex is in the third year of teaching undergraduate students in the clinical patient-care setting.

Alex identified that completing tasks, such as bathing and medications, as well as helping the nursing student grow and learn among their top priorities when working in the role of the clinical nursing educator. When asked about their perception of the role and responsibilities of a clinical nursing educator, Alex shared the following:

I'm responsible for making sure that they do the tasks that they [the students] are assigned. So from that standpoint, yeah, there is a list of A, B, C, D, and E, that I expect them [the students] to get done but then I also feel responsible for helping them to learn along the way as they're getting those things done that we expect them to do. [Such as] AM care and documentation and medications. Those are things that they have to get done. They are tasks that all nurses have to do. But then to help them learn, to help them grow, to help them see why those things are important.

Alex was unable to verbalize a particular teaching style use in the clinical setting. Rather, Alex indicated that their teaching style is dependent on the clinical day. Alex responded: "It's [their teaching style] rather fluid depending on the day, depending on the students and what they need, depending on me and how I'm feeling. So I don't know that I could put a particular style to it." 
When asked about a particular person(s) they emulate when teaching, Alex was able to recount experiences with particular former instructors that both positively and negatively affected their learning as a student nurse. Alex shared that, each of these past nursing instructors have been influential in modeling the role of a clinical nursing educator. The following passage was Alex's response to the question "Do you have a particular person you emulate when you are teaching?" I do. I have a couple of them.... a friend of mine was an instructor there [at the university she attended as an undergraduate] and students just seemed to love her and learn from her and she had such a heart to teach. She wanted students to learn and so she was fun but she was always on top of us. She knew what was going on with everybody and so I try really hard to pattern myself after her.

Alex also provided the following response concerning negative experiences with a clinical instructor from their undergraduate program:

...now I had that clinical instructor who just was about you doing the tasks and doing that... I got to do this and that. And it wasn't about the teaching environment or learning environment. It was, you are here to do this, this, and that.

Alex also shared that they draw additional teaching experiences from home schooling their children. When asked if the clinical rationale that they provide to the students in the clinical setting originates more from experience as a registered nurse or a nurse practitioner (NP), Alex provided the following response:

Probably more ... well I don't know. Maybe a combination, a little heavier on the NP side. Because some of that is what you gain in that role in that piece of education. It's that higher education where you gain that extra knowledge to be able to answer some of those questions. 
During the clinical observation sessions, Alex demonstrated several examples of utilizing experiences from their professional role when teaching undergraduate students in the clinical patient care area. As one example of this, between observation day two and observation day three, Alex volunteered to travel abroad on a university-sponsored mission trip in order to provide nursing care to patients at a medical clinic located in a third-world country. During this trip, Alex worked in the role of a NP in order to triage patients, provide medical care, complete physical assessments, and prescribe medications. When beginning the pre-conference session on observation day two, Alex began the session by recounting stories from the trip abroad pertaining to their experiences providing nursing care to adults and children with unique medical needs. Alex provided several examples from their trip in order to compare and contrast the modern health care system in the United States and that of the country they visited. During this discussion, the students were engaged and were able to ask questions pertaining to Alex's mission trip. Additionally, several students in the group also reflected upon and shared their experience in providing care or services to patients or individuals outside of the United States. By engaging the students and sharing professional experiences, Alex utilized the teaching practices of discussion and reflection to facilitate the students' learning.

\section{Bailey's Grounding Experiences}

Bailey has worked as a registered nurse for over seven years, predominantly caring for patients in the cardiovascular intensive care unit. Bailey worked as a Family Practice Nurse Practitioner for a year full-time before transitioning into to the role of clinical nursing educator. Bailey was completing their first full-time year of teaching during the observational sessions.

When asked if the clinical rationale that they provided to students when teaching in the clinical patient care setting was drawn more heavily from being a nurse practitioner or a 
registered nurse, Bailey provided the following response: "Honestly, it's a combination. I had, I pull a lot as far as skill-wise, but a lot of the actual like looking at labs, the why meds, the patho [pathophysiology], I feel like a lot of that comes from my NP.” Bailey also cited personal experiences, or experiences outside of healthcare, contributing to their teaching. Bailey stated the following when asked about prior experiences that have influenced their teaching:

I definitely draw from the different kinds of experiences that I've had, but I've even drawn from, it sounds weird, but retail experience when worked retail. So I pulled from that to be able to meet common ground with them [the nursing students] about what things were like, what their job was like and things like that. So I think honestly I try to use just about anything I can to connect with patients and to teach students to connect with patients, to show them.

When asked about perceptions of the role or responsibilities of a clinical nursing educator, Bailey identified doing as many tasks or skills as possible, facilitating student learning, and patient safety as priorities. Bailey shared the following:

Probably role and responsibilities to facilitate learning for the students in the clinical environment, so to help them learn as much as they can. So trying to, for me, trying to strike a balance between getting as many things done as they can and then taking the time to really teach with that, but also as a nurse to protect the patients and keep the patients safe. So kind of balancing, keeping the patient safe, with the students.

When asked to identify a particular teaching style used when teaching in the patient care setting, Bailey had the following response:

But I try to really break it down into a way that they will understand. And, I am big on, like imagery, so like explaining to them, so like drawing pictures for them. Um, I try to 
have them do things as much as they can, and then if they need assistance step in after that. So kind of letting them do it.

When asked about an influential teacher or person they emulate while teaching, Bailey identified peers or colleagues as positive influences in their teaching. However, Bailey verbalized that they also draw from experiences with a former middle school teacher with whom they had a very negative experience. Bailey’s response:

I had a teacher that I was terrified of when I was in middle school. I had several teachers I was terrified of. That made for a really rough year and just the fact that I didn't want to ask questions, I was like afraid to go to class. I did. I got good grades but I just don't want anybody to dread coming to clinical or feel belittled in clinical. I try really hard to be approachable to make sure that they [the students] feel comfortable asking questions. That it's a safe learning environment.

Drawing upon this negative interaction with a former teacher, Bailey identified being approachable and allowing the students to feel comfortable when working with them in the clinical patient-care area as very important and meaningful qualities or behaviors of a teacher. Bailey voiced they strive to create a safe learning environment that does not promote the students' feeling "afraid" or "belittled". Rather Bailey wants the students to feel "comfortable" and "safe" when working with and learning from them in the learning environment.

Bailey's negative experiences with a former teacher may actually be influential in their decision-making process and comfort level when providing negative or constructive feedback to clinical students. As evidence of this, during the first day of clinical observations, Bailey was meeting with clinical students for the first time. Bailey started the day by reviewing the rules and regulations of the clinical component of the course, including dress code and other school and 
course policies. According to these polices, students would receive a punishment, or a professional role violation, if they are in violation of these policies. As Bailey was explaining their expectations, they stated "At this point, you all know the dress code, school of nursing policies. I don't like the whole professional role violation thing, so please don't make me do it" As this example demonstrates, Bailey's previous experience of feeling "belittled" or "afraid" as a middle school student may be powerful and/or influencing force on them not wanting to provide negative feedback or punishment, and their desire to be approachable to students.

\section{Chris's Grounding Experiences}

Chris has over twenty-five years of experience as a registered nurse and over 19 years of experience as a family nurse practitioner. Chris has worked in a variety of specialty clinical areas including in women's health, hemodialysis, cardiac care, and in a neurosurgical intensive care setting. Chris was finishing their third year of clinical teaching during the observational sessions. When asked about prior professional experiences that influence their teaching, Chris reflected on experiences working with graduate nurse practitioner students. Chris stated:

Well, because I had some experience as a preceptor in the outpatient clinical setting, so I use some of that experience as far as, it was a Master's level, so I did have to differ my strategies at the level that I'm in now [the undergraduate level].

When asked about perceptions of the role or responsibilities of a clinical nursing educator, Chris offered the following simple reply: "To provide the students with a conducive learning environment." When asked about a particular teaching style, Chris felt that striving to stimulate the students to critically think in the patient care setting an important part of their role. Chris provided the following response: 
Let's see, how do I verbalize my teaching style? It's more of a, I try to strive[for] the students to critically think as opposed to just telling me the facts of why, when, and how. Definitely my style is to stimulate critical thinking without telling them the actual answer. When asked if, when teaching in the clinical setting, Chris drew more from experience as a registered nurse or from experience as a nurse practitioner, Chris provided the following response:

It's probably a little bit of both. I relate back to my experience as an RN working in various units and floors. I actually relate some of my teaching to when I was a student. There's some things I bring up when I was a student. Then some of the rationale, such as labs and more complex things they're learning, probably more of an NP level.

As an example of Chris drawing from experiences as a nurse practitioner, during a postconference session, Chris was listening to a nursing student provide information as part of a clinical assignment concerning the application of a source of heat, such as a heating pad, to a patients' skin. As part of the presentation, the student provided information pertaining to the limited length of time a source of heat could be safely be applied to the patient' skin. In response to this, Chris shared a story concerning an experience with a patient who had a negative clinical outcome related to prolonged exposure to head. Chris stated "When I worked [as a nurse practitioner in breast surgery] I had a patient who left a heating pad on too long" and then related a story about a patient who had extreme tissue and incisional damage caused by prolonged heat exposure. In making this response, Chris drew on experiences working as a nurse practitioner in a breast care clinic in order to provide the students with a real-world example and facilitate the students' understanding of this concept. 


\section{Dana's Grounding Experiences}

Dana has worked as a registered nurse for over thirteen years. Dana's experiences range from working in cardiac care units and in the emergency department. Dana is currently enrolled in their second year as a nurse practitioner student. During the observational sessions, Dana was finishing their first year as a clinical nursing educator.

Dana voiced that they draw on experiences both as a nurse and as an NP student when teaching in the clinical patient care area. When asked specifically if they draw more from their experience as a registered nurse or a nurse practitioner student, Dana responded: "I would say it's $75 \%$ from on the job [as an RN], 25\% from being a [NP] student. And that's from saying "Here's how I learned to learn"

When asked about a person or persons they emulate when teaching, Dana provided the following response: “Old, previous teachers. Previous teachers and colleagues. And some trial and error with, not educating students, but educating new nurses." Dana elaborated by providing several responses concerning drawing from experiences with educating new nurses in the clinical setting and how educating new nurses may differ from educating undergraduate nursing students. An example of one of Dana's responses is provided below:

So with a novice nurse it's more about physical strategies. I don't know if I want to say physical strategy, but the actual work order from a student it's more of concepts, I feel like. A novice nurse knows the concepts because they went through school. And then when they get on the job, my thing will say, "well here's some things that we do on the job that were some physical ways that I put in an NG or a physical way that I put an IV that works for me." And for a student, my job is to say, "here is why someone has an NG 
and here's how to do it." So it's more of the concepts or the cardiac concepts or abdominal concepts, those kind of things.

When asked about perceptions of the role and/or responsibility as a clinical nursing educator, Dana was the only participant to draw a connection between the clinical setting and the didactic or classroom setting. Dana stated "I feel like my role is to reinforce the things that they learned in the classroom and then get them prepared for the things that they will learn."

Similar to Bailey, Dana voiced that creating a non-intimidating or approachable learning environment was important to their method of teaching. The question posed to Dana "Do you feel you have a particular teaching style" yielded the following response:

I feel like my teaching style is I try to make a holding environment or a semipermeable membrane to where they're in a situation to where they feel challenged but they don't feel overwhelmed or stressed. So I try to make this nice little circle to where they get the challenge of taking care of a patient, but they don't feel like they have to do it all on their own and that they can get the difficulty of being a nurse without feeling that there's something that they don't know that they can't ask about."

During the second clinical day, Dana provided an example of how their professional experiences influenced their teaching practices. Prior to the clinical day, Dana developed two scenario-based case studies to be used during the post-conference session. To begin the postconference session, Dana introduced the learning activity to the clinical group. Dana called this activity "what to do when things go bad." Dana explained "both [case studies] are based on real life events that happened to me [when working as a registered nurse in the emergency department]". Dana provided the student group with patient-based scenarios with detailed clinical information, including patient assessment data and vital signs. Dana then asked the 
students to think about and verbalize nursing interventions they would implement first, what information is important, and what information they could overlook. By using this teaching practice, based on Dana's professional experience as a registered nurse, Dana was able to engage the students in discussion and facilitate their critical thinking.

\section{Summary of Grounding Experiences}

There were several similarities between and amongst the participants concerning their grounding experiences; however the participants also revealed differences in their responses and/or actions. The four participants had a wide variety of clinical experiences prior to transitioning to the role of novice clinical educators. It is through these professional working experiences that the participants developed their expertise as a clinical nursing expert. In addition to their professional experiences, their personal experiences as either undergraduate or graduate nursing students have also greatly influenced their teaching practices. Each participant voiced that they draw upon their past educational experiences with former professors, both positive and negative, when making decisions concerning their particular teaching style, facilitating student learning, and/or dealing with disciplinary issues. Likewise, the participants were similar in that they all drew upon a combination of their knowledge acquired as a registered nurse, a nurse practitioner, or nurse practitioner student when providing clinical rationale to nursing student in the clinical setting. When working with students in the clinical setting, I observed that each participant shared stories pertaining to their work as either a registered nurse, a nurse practitioner, or nurse practitioner student. These stories were told in order to provide real-world examples of nursing care, aid the student in making clinical connections, to develop original teaching content, and/or demonstrate sympathy for students who may be struggling to learn in the clinical setting. 
Although the participants identified and demonstrated many similarities in their grounding personal and professional experience, their past experiences also differed in several ways. For example, Dana and Chris voiced that their previous experience working with novice nurses or graduate students influenced their teaching practices. Alex identified home schooling their children and Bailey mentioned working in a retail job as a grounding influences on their teaching. Likewise, the participants differed in their perception of the role of the clinical nursing educator. Alex and Bailey each identified they believed that helping the students learn and complete tasks or "getting as many things done" as their primary role or responsibility as a clinical nursing educator. Bailey also mentioned "keeping the patient safe" as one their primary roles as a clinical nursing educator. Dana believe that making a connection between the clinical and didactic setting was their primary role. Chris, however, identified the creation of a "conducive" learning environment and stimulating critical thinking as their primary role as a clinical nursing educator.

\section{Summary of Section One}

This section provided an overview of the participants' professional experiences, examples of the grounding experiences of each participant, and the similarities and differences of these grounding experiences. Although the participants shared many of the same unique grounding experiences, they differed in their perception of their role and/or responsibilities when teaching undergraduate students in the clinical patient-care area. Chapter five provides a further discussion of these differences. The next section of this chapter provides a detailed account of the in-the-moment teaching practices used by the each of the individual participants when teaching in the clinical setting as well as a summary of teaching practices common to the 
participant group as a whole. Additionally, an account of how the participants changed those practices in relation to the real-world clinical context is provided.

\section{Section Two: Teaching Practices used in the Clinical Patient Care Setting}

The second aim of this research study was to investigate the underlying knowledge and teaching practices used by clinical experts who transition into the role of the novice clinical educator. The purpose of this section is to provide findings related this aim. The Pedagogical and Subject Matter Knowledge for Clinical Nursing Educators framework, adapted from the Mathematical Knowledge for Teaching (Ball et al., 2008) was used to provide the guiding framework for which each teaching practice or unique source of knowledge is correlated. Like the original Mathematical Knowledge for Teaching framework, the adapted Pedagogical and Subject Matter Knowledge for Clinical Nursing Educators framework is divided into two sections: Subject Matter Knowledge and Pedagogical Knowledge. Pedagogical Content Knowledge is used to identify the type or purpose of the observed teaching practice and includes the following themes: Knowledge of Content and Students, Knowledge of Content and Teaching, and Knowledge of Content and Curriculum. These themes are used to describe the unique teaching practices that clinical nursing educators demonstrated when teaching in the clinical setting. There are three themes that fall under Subject Matter Knowledge: Common Content Knowledge, Specialized Content Knowledge, and Horizon Content Knowledge. These themes are used to describe the unique knowledge that the clinical nursing educator drew upon when teaching in the clinical setting. Findings in this section have been organized by these theoretical themes as well as by the distinctive patterns of communication, responding and questioning, that were used by the participants during the investigation. Observation and ethnographic interviewing conducted during the pre-and post-conference sessions as well as during the clinical 
day were data collection methods used to identify and describe the teaching practices used by the participants.

\section{Pedagogical Content Knowledge}

This section includes a discussion related the Pedagogical Content Knowledge of the participants. This includes the findings particular to themes used to describe the participants' knowledge of curriculum, students, and teaching as well as correlating teaching practices. An explanation of the themes, teaching practices, and key examples of each teaching practice are provided below. Additionally, information pertaining to which participant(s) primarily used these teaching practices and the context in which the participants demonstrated each of these teaching practices is provided.

\section{Knowledge of Content and Curriculum}

According to Ball et al.'s (2008) Mathematical Knowledge for Teachers, Knowledge of Content and Curriculum (KCC) includes a teacher's understanding of the school or course curriculum. KCC may also include the teachers' knowledge of what is to be taught and in what particular order it should be taught (Judson \& Leingang, 2016). Shulman (1986) suggests that a teachers' curricular knowledge encompass their understanding of now only the curriculum particular to their course, but also content that have been and will be taught in during the preceding semesters, during the concurrent semester, or in future semesters. For clinical nursing educators, $\mathrm{KCC}$ includes the clinical nursing educators' knowledge of course and program objectives, specific clinical objectives, and clinical assignment objectives. Additionally, this would include the clinical nursing educators' knowledge of appropriate skills and nursing interventions the nursing student can perform or not perform independently or with supervision based on their academic level in the nursing program. 
The participants in this investigation provided evidence of their $\mathrm{KCC}$ through their teaching practices. These teaching practices included: providing directions for completion of assignment, setting expectations for the clinical skills and tasks the students could or could not complete, and asking the students questions pertaining to their understanding of how to collect data for and/or complete clinical assignments. Examples of each of these teaching practice follows.

Responding: Providing Directions for Completion of Assignment. All of the participants drew upon their KCC pertaining to their individual courses when providing the students with information related to the curricular requirements for completing each assignment that was required as part of the clinical component of the nursing course. These assignments included care plans, preplans, pathophysiology trees, and patient teaching projects. Based on their KCC, the participants provided the following information to the students: requirements for project completion including appropriate and inappropriate clinical data to be collected, project due dates, required formatting (such as APA), feedback on appropriate or inappropriate patients for which to gather assessment data, common mistakes made by students on particular assignments, and explanations and rationale related to grading of the project. Several examples of how the participants utilized teaching practices related to their KCC follow.

During a preconference session, Chris was reviewing expectations with the clinical group related to the citation requirements for a preplanning assignment. When speaking to the clinical group, Chris provided the following information based on their knowledge of the requirements of the assignment: "one of the most common mistakes was APA format on the reference list. If you guys can [please] write the edition. We have to make sure that if I look at the reference list, I can find the edition." 
In a separate example, during a post-conference session, Bailey provided direction and information to the clinical group detailing expectations for completion of a pathology tree assignment. During this exchange, Bailey stated "labs and medications. Those are two big things I will look for." Bailey then provided additional information concerning completion of the assignment by stating "You don't have to include everything. One page. You should fit everything on one page."

\section{Questioning: Asking Questions Concerning Completion of Assignment. The} participants also demonstrated KCC when asking questions in order to determine the students' understanding of how to complete an assignment, the rationale behind the students' patient selection, and/or determining the students' understanding of how they would be graded on the project. The participants used these teaching practices primary in the pre-and post-conference sessions. Often, they would either begin the clinical day or conclude the clinical day by asking the students if they had any questions pertaining to the completion of an assignment. They would then follow up this question with information or direction on how to correctly complete the assigned based on their KCC.

As an example of this, during the preconference session, Dana questioned the clinical group about the completion of a clinical pathology assignment. Dana stated to the group "Did anyone do their situational patho?" The students and Dana then engaged in a brief discussion concerning the completion of this assignment for which Dana replied "Don't forget, you just need to add a citation to the end [of the assignment]."

\section{Responding: Setting Expectations for the Completion of Skills or Task. The} participants demonstrated their KCC by communicating their expectations for what skills or tasks the student could or could not perform safely and competently in the clinical patient-care 
area, based on their academic level in the nursing program. The context in which the participants used their KCC to establish and communicate their expectations for students' completion of a skill or task varied during the clinical day. Most often, the participants shared their expectations with the student group during the pre-conference sessions. The participants also commonly shared this information with the students throughout the clinical day.

For example, during the clinical day, Alex was approached by a registered nurse working on the clinical unit who inquired as to whether a particular student was able to collect a urine sample from their assigned patient. Based on their KCC, Alex's response to the nurse and the student was "she can't draw blood but she can catch pee." This statement demonstrates Alex's understanding of the clinical skills that the student is competent in completing (i.e. collecting a urine sample), and those that the student is not yet prepared to complete, such as performing venipuncture in order to draw blood samples.

In summary, all of the participants utilized their $\mathrm{KCC}$ to provide the nursing students with directions for completion of course requirements. Additionally, they utilized this knowledge when asking questions in order to assess the students' understanding of how to complete required assignments. Finally, the participants used this knowledge when setting student expectations or communicating with other nursing staff what skills, tasks, or nursing interventions the students could or could not complete during the clinical day based on their academic level.

\section{Knowledge of Content and Students}

According to Ball et al.’s (2008) Mathematical Knowledge for Teachers, Knowledge of Content and Students (KCS) is a combination of knowledge that includes knowing about students and knowing about the discipline (Ball et al., 2008). Central to this construct is the teachers' knowledge of student conceptions and misconceptions, a teachers' ability to anticipate 
what a student may think or know, and what they may find confusing, and what examples to choose to facilitate a students' learning (Ball et al., 2008). KCS requires a teacher to have a deep understanding of not only the content they are teaching, but also how students may learn and/or come to understand the content (Ball et al., 2008). Based on the Pedagogical and Subject Matter Knowledge for Clinical Nursing Educators framework, KCS also includes the clinical nursing educator's understanding how nursing students learn or come to understand concepts in the clinical patient-care area. This may include the selection of specific examples used in the patient care area in order to clarify or explain a concept. It also includes the clinical nursing educators' ability to anticipate what a nursing student may understand as well as common misconceptions.

The participants in this investigation demonstrated two unique teaching practices for which they drew upon their KCS. These were: using examples and clarification of misconceptions or misunderstandings. These teaching practices involved providing responses to students; however they did not accomplished this through the use of questioning. While all four of the participants used at least one of these teaching practices, only Chris and Dana used both of these teaching practices when teaching in the clinical patient-care area. An explanation and an example of each teaching practice is provided below.

Responding: Using Unique Examples. When teaching in the clinical setting, based on their KCS, the participants utilized unique examples in order to facilitate the student's understanding or interpretation of difficult concepts, to clarify common misconceptions, or to facilitate the completion of tasks or skills. Typically these unique examples were provided during on-the-spot teaching-learning sessions on the clinical unit, and/or during the pre-conference. The following example, provided by Chris, demonstrates the use of this teaching practice. 
During the clinical day, Chris was observed working with a student who was preparing to complete glucose testing on a patient. The student, a second-semester sophomore, had performed this skill only one time prior to this clinical day. In order to complete this skill, the student was required to place a small amount of blood onto the end of a glucose test strip, via syphon-like method, at a 45-degree angle. It is common, for students at this academic level, to misunderstand how to correctly angle the patient's finger at a 45-degree angle. Therefore, prior to the student performing the skill, Chris stated to the student "pretend like you are diving in a pool" to describe the process and the angle needed to correctly place the blood onto the strip. Chris based this teaching practice, using unique examples, on their knowledge of nursing practice, as well as on their knowledge of this particular level of student and their common misconceptions or misunderstandings related to performing this skill. Through this teaching practice, the student was able to understand the correct procedure and correctly complete the skill.

Responding: Clarification of Misconceptions or Misunderstandings. During the preconference sessions and the clinical day, the participants often provided responses, based on their KCS, to clarify students' misconceptions and/or misunderstandings. This teaching practice was used to provide students with direction for care, to facilitate the students' understanding of laboratory results, and to provide the correct pronunciation of words or medical terminology.

As an example of this teaching practice, during the preconference sessions, all of the participants provided clarification of mispronounced words or misunderstood concepts to students. This included the correct pronunciation of the words "arthroplasty" and "residual" in addition to numerous other medical and nursing terminologies and concepts. As an additional example, during a short interview session on the clinical unit, Chris revealed to the researcher that they had experienced several sophomore-level students incorrectly utilize their prepared 
paperwork to reference which medications were to be administered to the patient, rather than utilizing the correct practice of referring to the MAR (Medication Administration Record) found in the Electronic Medical Record (EMR). Based on Chris's observations and understanding of this common misconception, Chris anticipated the student's potential actions. Prior to medication administration, Chris gently reminded the student to "look at the MAR, and not your paperwork" in order to prompt the student of the correct procedure for medication administration. The use of this teaching practice was derived from Chris's understanding of, not only the students' common misconceptions, but also their professional knowledge of the correct and safe process of administering medications.

In summary, the participants used their KCS to provide unique "real-world" examples in order to facilitate student learning of concepts that may be difficult. Additionally, this knowledge was used in order to facilitate the students' understanding of how to safely and correctly complete nursing skills. Finally, all the participants drew on this knowledge when helping students to correctly pronounce new words from the medical and nursing vernacular that were unfamiliar to the student.

\section{Knowledge of Content and Teaching}

Knowledge of Content and Teaching (KCT) combines knowledge of the discipline and knowledge of teaching and strategies to guide student learning (Ball et al., 2008). This construct may include the following teacher responsibilities: designing instruction, choosing the sequence of content presentation or discussion, selecting of examples, choosing a particular teaching method, and discriminating the advantages and disadvantages of using particular representations to teach a specific idea or concept. KCT requires an interaction between specific content understanding and an understanding of pedagogical methods that facilitate students' learning 
(Ball et al., 2008). In clinical nursing education, KCT includes the clinical nursing educators' knowledge of specific teaching practices that may be used to facilitate learning and critical thinking in the clinical patient care area. KCT includes the clinical nursing educators' ability to choose the correct or appropriate teaching method for in-the-moment instruction of students in the clinical patient-care area. As the clinical patient-care area is a very distinct learning environment, much like the classroom in the didactic setting, KCT for clinical nursing educators also incorporates constructs from Shulman's (1986) general pedagogical knowledge including classroom management and organization and classroom communication and discourse (GessNewsome \& Lederman, 2001).

When teaching in the clinical patient care setting, the participants demonstrated numerous examples of teaching practices, including an array of responses and types of questions asked of students, as evidence of their KCT. This included the use of the following teaching practices when responding to students: teaching through personal stories, demonstration or role modeling, and the creation of original learning materials. The participants used the following teaching practices when questioning students: asking for student reflections, asking low-level knowledge or recall questions, and asking questions that stimulate critical thinking. Classroom management is a final teaching practice identified in this theme. A brief description of each teaching practice, where and when the teaching practice was used, and an example of each is provided below.

Responding: Teaching Through Personal Stories. As discussed in the first section of this chapter, the participants often draw on their personal and professional experiences when teaching students in the clinical patient' care area. These stories are used to facilitate students' understanding of difficult concepts, and/or to provide real world examples. In this investigation, 
each participant used the pedagogical practice of teaching through personal stories in the pre-and post-conference settings as well as in the patient-care setting.

As an example of this, during the preconference setting, Alex was facilitating a discussion with students concerning the medical and nursing management of a patient who was demonstrating symptoms of a urinary tract infection (UTI). As part of this discussion, Alex stated the following: "This is just a life observation, residents often overlook more simple issues, such as a UTI, and, instead, use expensive exams to try and diagnosis something else. They [residents] order a lot of medical exams before they consider the obvious." Alex then led their students in a detailed discussion of nursing assessment and treatment of a urinary tract infection. During this exchange, Alex combined knowledge of the discipline, as both a registered nurse and nurse practitioner, in order to facilitate the students' understanding of the care required for the patient with a medical diagnosis of a UTI.

Responding: Demonstration or Role Modeling. All of the participants were observed utilizing demonstration or role modeling during as a teaching practice in order to facilitate student learning in the clinical setting. Most often, this teaching practice was used to guide students during the clinical day in performing nursing interventions, such conducting a physical assessment, utilizing or operating various pieces of medical equipment, such as IV pumps, Doppler devices, hospital beds, or accu-check machines. This teaching practice was also commonly used to guide students when preparing medications for various routes of administration, including subcutaneous and intravenous injections, and via a gastric tube. An example of this teaching practice follows.

When working with a student to prepare and administer a subcutaneous injection, Alex used the teaching practice of demonstration, along with verbal prompting, in order to explain the 
correct technique for administration. As part of this explanation, Alex put their arm out in front of the student and pinched the skin in order to demonstrate to the student the proper technique of bunching or pinching the skin prior to the injection. By providing the students with this in-themoment demonstration, Alex facilitated the students' understanding of the correct method of injection for this medication.

Responding: Creation of Original Learning Materials. Three of the participants, Alex, Bailey, and Dana, were observed using student-centered learning materials or activities that they developed in order to facilitate learning in the clinical setting. These learning activities, implemented during the pre-and post-conference sessions as well as during the clinical day, were used in order to provide an explanation or clarification of a particular concept, disease process, or nursing skill. This teaching practice included the utilization of visual pictures, short teaching sessions, or other clinical educator-created learning activities.

As an example of a clinical nursing educator-created learning activity, during the preconference session, Dana provided a list of drafted questions for each student, based on their specific patients' condition or medical diagnosis. Dana provided the group directions for the activity by explaining that, during the clinical day, each student was required to research and answer the list of provided questions. During the post-conference session, Dana asked the student to reflect on the findings of or answers to these questions. Dana then used these question to engage the individual student as well as the clinical group in discussion.

As an additional example, during the preconference session, Alex was observed approaching a student and handing them a piece of paper with, what appeared to be, a large picture of the human heart with words and arrows pointing to various aspects of the anatomy of the heart. When asked about this, Alex revealed that, prior to the clinical day, they thought about 
this particular student and the patient they would be caring for during the clinical day. This patient had been recently diagnosed with the disease process of congestive heart failure (CHF). The student, a second-semester sophomore, had not yet received didactic content concerning the disease process and nursing care related to a patient diagnosed with CHF. However, Alex shared with me that they wanted the student to have an understanding of the disease process of CHF in order to safely provide care for the patient. Based on their knowledge of the disease process, Alex developed and provided the student with a visual picture of heart in order facilitate the students' understanding of the disease process of CHF. In addition, this was also done in order to facilitate the students' recognition and assessment of signs and symptoms of left- and right-sided heart failure when caring for the patient.

Responding: Classroom Management. Knowledge of Content and Teaching also includes the clinical nursing educators' knowledge of and ability to organize the classroom, including classroom communication (Gess-Newsome \& Lederman, 2001; Shulman, 1986). The knowledge required to manage student learning in the clinical patient-care setting is unique and different from the knowledge required to organize and manage student learning in the traditional classroom setting. In the clincial settting, the participants drew upon their knowledge of the dicipline and teaching by demonstrating the following elements of classroom management: determining which patients on the clinical unit the students were assigned to care for during the clinical day, providing directions to student related to the overall schedule for the clincial day, facilitating the students' completion of tasks in a timely manner, including nursing interventions and administration of medications, and supervising all aspects of nursing care delivered to the patient by the nursing students. The participants used classroom management practices during the pre-and post-confernce sessions, as well as numerous times throughout the clinical day. The 
following section provides an example of Bailey's utlization of the teaching practice of classroom management.

During the first clinical observation day, Bailey was observed providing directions and clincial rationale to a nursing student who was ordered to administer insulin to an assisgned patient. During the exchange with this student, Bailey was approached at the same time by two additional students who verbalized concerns for the care of their assigned patients and needed Bailey's assistance. After taking a brief moment to consider the three students' requests and the needs of all three patients, Bailey turned to the first student and stated "ask the nurse if she will give the new meds, and we will give the rest." Bailey then turned to the second student and stated "let me do meds [with this student] first, and then we will do it [change the central line dressing] next." Bailey then returned to giving medications with the original student. In this example, Bailey drew upon knowledge of the discipline of nursing, including prioritization and organization of patient care, in order to communicate to and direct the care of multiple nursing students.

Questioning: Engaging in Discussion and/or Reflection. In previous sections of this chapter, several examples have been provided to describe when and where the participants used various other teaching practices, such as teaching through personal stories, and the creation of original learning materials, in order to engage the students in discussion. As a participant group, the participants were rarely observed during the clinical day engaging the students in discussion. Dana, Alex, and Chris were observed using discussion as a teaching practice primarily in the preand/or-post conference settings. Bailey was not observed routinely engaging their students in discussion in any of the settings. 
All of the participants used the teaching practice of engaging students in reflection. This teaching practice was, primary, used by the participants in the post-conference sessions and, at times, during the pre-conference session. Like discussion, the teaching practice of engaging the student in reflection was seldom used by any of the participants during the clinical day. Participants who used this teaching practice in the post-conference sessions did so in order to engage the students to reflect on the following themes or concepts: the interventions or skills that were completed during the day, procedures that the students were able to observe during the clinical day (e.g. PICC [peripherally inserted central catheter] line insertion), and/or their overall perception of the care they provided during the clinical day. The participants prompted the students to reflect on their experiences by stating, "how was it," "what did you see, "or "how did it go?" This teaching practice was rarely used by any of the participants to engage in higher-level thinking or to connect concepts from the classroom to the clinical setting. Questioning that may have been used to engage higher level thinking may have included questions such as "what are the risks and/or benefits for a patient with a PICC line?" or "how would the patients' plan of care change now that they have a PICC line?" The period of facilitating student reflection lasted anywhere from a few moments to more than half of the post-conference session. Bailey and Chris routinely provided the students with the least amount of time for reflection, whereas Dana and Alex provided the most. An example of the teaching practice of engaging students in reflection was observed during Dana's pre-conference session.

During the pre-conference session, Dana and the student group were discussing the patients they would be caring for during the day. During this discussion, one of the students expressed that the patient for which he would be providing care this day had been admitted to the hospital for a colostomy reversal. To this statement, Dana posed the following question, "why do 
you think he would want his colostomy reversed?" Dana allowed the student to think and respond to this question. Dana then followed up and posed the question "If you had the option and you had a colostomy would you want to have it reversed?" The student thought for a moment, and then responded by empathizing with the patient and expressing how he would feel if he had a colostomy. Several of the other students in the group asked questions along with also sharing their thoughts, ideas, and fears of having to wear a colostomy appliance. In using this teaching practice, Dana was able to engage the students and allow them to think empathetically and reflect on ways to provide appropriate nursing care to this patient.

Questioning: Asking Knowledge and/or Recall Questions. One of the most common teaching practices used by all of the participants was asking the students' particular questions in order to engage their previous knowledge and/or asking them to recall facts, numbers, or other basic information. These questions were primarily asked during the pre-conference sessions and during the clinical day. The questions were asked by the participants in order to ascertain the students' understanding or knowledge of the following medical and/or nursing concepts: principles of medication administration including drug class, route or method of administration, and common side effects, nursing interventions, disease processes, and interpretation of laboratory results. The questions posed by the instructor were most often answerable with a "yes," or "no," or a short reply from the student. The participants prompted students to provide an answer to their knowledge or recall question by asking the students "what is," "tell me about," "how do you," "do you know," and/or "what do we call." The following is an example of Baileys' utilization of this practice in the clinical setting. 
During the clinical day, Bailey was observed working with a student who was preparing to give the medication Lovenox [an anti-coagulant] via a subcutaneous injection. The following exchange between Bailey and the student occurred prior to the administration of this medication:

Bailey: Where are we going to give [the injection site] Lovenox?" The student responded.

Bailey: "How far from the umbilicus?" The student responded.

Bailey: "How do we engage the safety?" The student responded.

Bailey: "What do we do with the air bubble?" The student responded.

Upon conclusion of this line of questioning, Bailey and the student entered into the patients' room to administer the medication. As this example demonstrated, Bailey used the teaching practice of asking knowledge or recall questions for which the student utilized their basic knowledge of the medication Lovenox in order to answer.

Questioning: Asking Application or Critical Thinking Questions. Asking questions for which the nursing student would need to apply concepts or think critically to answer was a final teaching practice that each instructor was observed using when teaching in the clinical and/or the pre-conference setting. The participants prompted the student to provide an answer to these higher-level application or critical thinking questions by beginning the question with a "what" or "why" statement. These questions routinely were asked to simulate the students' application or critical thinking related to principles of medication administration, nursing interventions, and disease processes. Although the teaching practice of asking application or critical thinking questions was used by all of the participants, it was used far less by the participants than the teaching practice of asking knowledge or recall questions. Additionally, these questions were asked more frequently when working with students in the in the pre-and 
post-conference settings, compared to the clinical setting. The following is an example of Chris' utilization of this teaching practice in the clinical setting.

During the clinical day, Chris was observed working with the clinical students to complete accu-checks in order to inform their decision as to which patients would require insulin coverage. When working with a particular student, who had just reported a high blood sugar and informed Chris that the patient would require insulin coverage, Chris asked the student about the arrival of the patients' lunch tray. The student responded. Chris followed up with the question “why do we get concerned about the lunch tray?" in order to facilitate the students' critical thinking and application of their knowledge of different concepts (i.e. the patient's blood sugar level, the required dose of insulin, and the timing of the patients' lunchtime meal) needed to safely care for this patient.

In summary, the participants used their Knowledge of Content and Teaching in a variety of ways during the pre-and post-conference sessions and during the clinical day. These teaching practices were used to teach nursing students about skills, engage students in discussion, manage the needs patients in the real-world clinical area, elicit the application of prior knowledge, and facilitate critical thinking. In addition, three of the four participants drew upon their Knowledge of Content and Teaching in order to develop original teaching materials.

\section{Summary of Pedagogical Content Knowledge}

In summary, the participants in this investigation drew upon their knowledge of students, curriculum and teaching as well as from their knowledge of the discipline of nursing when using a variety of teaching practices in the clinical, pre-conference, and post-conference settings. As described in this section, not all participants were observed utilizing each identified teaching practice. Likewise, some teaching practices were used more predominantly in certain settings or 
by particular participants than others. A summary of the context in which the participants utilized the facets of Pedagogical Content Knowledge is provided in table 4.1 below.

Table 4.1

Context of the Use of Pedagogical Content Knowledge

\begin{tabular}{|c|c|c|c|}
\hline Pedagogical Content Knowledge & $\begin{array}{l}\text { Pre- } \\
\text { conference }\end{array}$ & $\begin{array}{l}\text { Post } \\
\text { Conference }\end{array}$ & $\begin{array}{l}\text { Clinical Patient } \\
\text { Care Area }\end{array}$ \\
\hline \multicolumn{4}{|l|}{ Knowledge of Curriculum } \\
\hline $\begin{array}{l}\text { Providing directions for completion } \\
\text { of assignment }\end{array}$ & $\mathrm{x}$ & $\mathrm{x}$ & \\
\hline $\begin{array}{l}\text { Asking questions concerning } \\
\text { completion of assignment }\end{array}$ & $\mathrm{x}$ & $\mathrm{x}$ & \\
\hline \multicolumn{4}{|l|}{ Knowledge of Students } \\
\hline $\begin{array}{l}\text { Setting expectations for completion } \\
\text { of skills or task. }\end{array}$ & $\mathrm{x}$ & $\mathrm{x}$ & \\
\hline Using unique examples & $\mathrm{x}$ & $\mathrm{x}$ & \\
\hline $\begin{array}{l}\text { Clarification of misconceptions or } \\
\text { misunderstandings }\end{array}$ & $\mathrm{x}$ & & \\
\hline Classroom management & $\mathrm{x}$ & $\mathrm{x}$ & $\mathrm{X}$ \\
\hline \multicolumn{4}{|l|}{ Knowledge of Teaching } \\
\hline Teaching through personal stories. & $\mathrm{x}$ & $\mathrm{x}$ & $\mathrm{X}$ \\
\hline Demonstration or role modeling & & $\mathrm{x}$ & \\
\hline $\begin{array}{l}\text { Creation of original learning } \\
\text { materials }\end{array}$ & $\mathrm{x}$ & $\mathrm{x}$ & \\
\hline $\begin{array}{l}\text { Engaging in student discussion } \\
\text { and/or reflection }\end{array}$ & $\mathrm{x}$ & $\mathrm{x}$ & \\
\hline $\begin{array}{l}\text { Asking knowledge and/ or recall } \\
\text { questions }\end{array}$ & $\mathrm{x}$ & $\mathrm{x}$ & $\mathrm{x}$ \\
\hline $\begin{array}{l}\text { Asking application or critical } \\
\text { thinking questions }\end{array}$ & $\mathrm{x}$ & $\mathrm{x}$ & $\mathrm{x}$ \\
\hline
\end{tabular}

The following section provides a discussion related to the knowledge and teaching practices that correlate to the other section of the Pedagogical and Subject Matter Knowledge for Clinical

Nursing Educators framework, Subject Matter Knowledge.

\section{Subject Matter Knowledge}

This section includes a discussion related the findings particular to the Subject Matter Knowledge of the participants. Subject Matter Knowledge is critical to the teaching-learning 
process and includes the knowledge of the content of a subject (Grossman, 1990). Subject Matter Knowledge may be used to describe the unique knowledge that the clinical nursing educator draws from when teaching in the clinical setting. This section includes a discussion of findings particular to themes used to describe the participants' Common Content Knowledge, Specialized Content Knowledge, and Horizon Content Knowledge. An explanation of the themes, teaching practices, and key examples of each teaching practice are provided below. Additionally, an explanation regarding which participants primarily used these teaching practices as well as how or when each of these teaching practices were used is provided

\section{Common Content Knowledge}

According to the Mathematical Knowledge for Teachers framework (Ball et al., 2008), Common Content Knowledge (CCK) involves recognizing when students provide incorrect information, understanding definitions common to the discipline, and the ability to perform the tasks or know the information that they expect their students to know (Ball et al., 2008). CCK may be further described as "knowledge of a kind used in a variety of settings; in other words, not unique to teaching" (Ball et al., 2008, p. 399). For clinical nursing educators, CCK includes the knowledge related to the discipline of nursing. It is the underlying nursing knowledge that enables the clinical nursing educator to facilitate the students' learning and/or patient care in the clinical patient care area. This includes knowledge of disease processes, medications, nursing interventions, and interpretation of laboratory values. Additionally, this knowledge is inclusive of the clinical nursing educators' ability to apply their experience as a clinical nurse when teaching in the patient-care setting, including the utilization of clinical judgment and critical thinking skills. This also includes recognizing when a student is providing incorrect clinical 
information. CCK would have been acquired during clinical nursing educators' undergraduate or graduate nursing education and through clinical practice.

When teaching in the clinical patient care setting, participants were observed consistently drawing upon their CCK in order to answer questions and provide responses to the nursing students. In fact, the participants were observed drawing upon their CCK more frequently than the other two types of Subject Matter Knowledge, Specialized Content Knowledge and Horizon Content Knowledge. All of the participants used their CCK in all settings. Because the students under their charge are novice nursing students, the participants were ultimately responsible facilitating nursing students' provision of safe and competent nursing care. Therefore, it was imperative to the safety of the patient that the clinical nursing educators were able to draw upon their CCK when providing clinical rationale, making clinical decisions, using their clinical judgment, and providing direction for care. Additionally, the participants were observed drawing upon their CCK in order to direct their questioning of students in order to ascertain additional information, to clarify clinical information, and/or question students about completion of tasks. A brief description of each of these facets which comprised the clinical nursing educators' CCK, as well as an example of how this knowledge was used in practice, is provided below.

Responding: Providing Clinical Rationale. The participants were observed on numerous occasions utilizing their $\mathrm{CCK}$ in all settings in order to provide clinical rationale, or a detailed clinical explanation as to what the students were hearing, reading, or seeing in the clinical setting. This clinical rationale was offered by the participants in order to facilitate the students' understanding of complex patient care needs or other real-world clinical issues, clarify incorrect information, explain the cause and effect of medications, and/or to describe signs and symptoms of disease processes. The following example, provided by Alex, demonstrates their 
ability to interpret and clarify a students' incorrect information and then provide the appropriate clinical rationale.

During the pre-conference session, a student reported to Alex that their patient was prescribed the medication Methotrexate to treat the pain that was caused by the autoimmune disease process of Rheumatoid Arthritis (RA). Alex immediately recognized that the student was incorrect, as although this medication may be indicated in the treatment of this disease, it is not indicated to treat pain. Alex questioned the student, again, concerning the indication of the medication, in order to clarify the students' understanding. The student provided the same incorrect response. At this time, drawing upon their CCK, Alex explained to the student and the clinical group that methotrexate is a medication used to treat the patient's disease process of Rheumatoid Arthritis, and not to treat pain. Alex provided a brief review of the disease process of RA, including the pathophysiology of the disease, as well as the correct clinical rationale for the use of this medication.

Responding: Using Clinical Judgment. All of the participants were observed numerous times drawing upon their CCK in order to make clinical judgments concerning patient care, administration of medications, and implementation of nursing interventions. This knowledge was used in the pre-conference and the clinical setting in order to assist students in their planning and implementation of patient care. For example, Dana was observed assisting a student who was preparing to administer medications to their assigned patient. As he reviewed the ordered medications, the student reported to Dana their concerns for administering an extended release anti-hypertensive medication. The student shared with Dana that the patient's current blood pressure was "low." After discussing and considering all of this information, Dana replied to the student "I would say, 99\% of the time I would give it [the extended release medication]." In this 
example, Dana drew upon their nursing experience and clinical judgment when considering all aspects of this clinical issue, including the type of medication ordered, the patients' vital signs, and the underlying reason the medication was prescribed, in order to advise the student to safely and appropriately administer the medication to the patient.

Responding: Providing Direction for Care. The participants were observed using their CCK in order to provide the students with directions for patient care in both the pre-conference session and during the clinical day. These directions were provided to students in order to facilitate their completion of nursing interventions or medication administration. Dana provides the following example of using this knowledge to direct the nursing students' care in the clinical setting.

In the clinical setting, Dana was working with a student who was responsible for administering a feeding solution and medications via a gastric tube. Prior to completing this skill, Dana stated to the student "Whenever you go in to do your assessment this morning, stop the feeding for about 15 minutes." Dana then provided the student further clinical direction on the correct technique for administering medication and feeding via a gastric tube. By drawing upon their CCK, Dana provided direction to the student in order to safely administer medications and feeding to the assigned patient.

Questioning: Asking for Additional Clinical Information. All of the participants drew on their CCK during in the pre-conference and clinical settings in order to ask questions and/or clarify information that the students were reporting. The use of questioning primarily centered on asking the student for additional information about the patient' clinical presentation including: current vital signs, pain rating, medications including the last provided dose, laboratory values, and assessment criteria. As an example, during the pre-conference setting Alex was observed 
listening to a student provide a report on the patient she was to care for during this clinical day. As the student provided information, Alex asked the student to stop the report and posed the following questions in order clarify the information they were getting from the student:

Alex: "What dose of medication is he on? What medication does he take?" The student responded.

Alex: "So the last time you looked at her pain rating, what was it?" The student responded.

Alex: "So the pain is controlled with the medication?" The student responded.

The student then provided additional information about the patient. In this exchange, Alex was observed drawing upon their nursing knowledge and used low-level questioning in order to ask for additional clinical information and to clarify the information provided by the student.

Questioning: Asking about Completion of Tasks. The use of questioning to ask about the students' completion of task was the most common type of questioning used in the clinical setting by all of the participants. Primarily, this line of questioning was used at the end of the clinical day in order for the participants to ascertain what nursing skills, interventions, or assessments had or had not been completed during the clinical day. Provided below are examples of questions from each participant used to ask their clinical student(s) about the completion of tasks.

Chris: "Did you do your neuro assessment?"

Alex: "Are there any interventions that you have been able to implement today?"

Dana: "Did you put a dressing over it?"

Bailey: "How are you doing? Did she [the patient] get cleaned up [bathed]"? 
Drawing on their nursing knowledge was well as their experience in caring for patients, all of the participants asked questions in order to understand what tasks or skills had or had not been completed by the students during the clinical day.

In summary, Common Content Knowledge comprises an inherent understanding of the discipline of nursing, including an understanding of concepts related to patient care, an understanding of when tasks or interventions must be completed during the clinical day, and recognizing when a student is providing correct or incorrect clinical information. The participants in this investigation also drew on this particular knowledge and their own clinical judgment in order to provide the students with clinical directions and rationale. Additionally, in using this knowledge, the participants were able to ask questions in order to clarify information and ascertain what nursing interventions had or had not been completed during the clinical day.

\section{Specialized Content Knowledge}

Specialized content knowledge (SCK) is based Shulman's (1986) original concept of subject matter knowledge (Hill, Ball, \& Schilling, 2008). Ball, Hill, and Bass (2005) conceptualized specialized content knowledge to mean "the mathematical knowledge that allows teachers to engage in particular teaching tasks..." (p. 377). SCK is particular to teaching, may be inclusive of everyday tasks, and is typically a knowledge set that is not needed or used outside of the realm of teaching. For the clinical nursing educator, Specialized Content Knowledge (SCK) is inclusive of the unique knowledge that the clinical nursing educator utilizes when teaching nursing students to care for patients in a clinical patient-care area. This knowledge would be specific to clinical unit policies, procedures, schedules and routines, the role of health care professionals, the role and responsibilities of the registered nurse working in a clinical patientcare area, and the everyday tasks of the registered nurse including medication administration and 
documentation in the Electronic Medical Record (EMR). SCK also includes knowledge of the nursing process. The clinical nursing would have, ideally, acquired SCK through clinical practice as a registered nurse.

The participants drew upon their SCK when providing information or asking questions involving the students' nursing care, nursing interventions, safe use of medical equipment, medication administration, and/or documentation of information. Additionally, the participants drew upon their SCK in order to provide nursing care that was required to be performed in a manner that was consistent with the specific protocols, standards, and/or the every-day operations of the clinical unit and/or acute care facility in which the instructor and students were working. Finally, this knowledge involved understanding the particular roles and responsibilities of other healthcare professionals, such as nursing assistants and therapist, on the clinical unit. Similar to their use of Common Content Knowledge, the participants drew upon their SCK in the pre-and post-conference setting, but most often in the clinical setting in order to provide the students with clinical rationale, directions for care, when utilizing their clinical judgment, and when asking the students about completion of tasks. The following provides a summary of each of these facets of SCK.

Responding: Providing Clinical Rationale. The participants drew upon on their SCK when providing clinical rationale that was used to facilitate the students' understanding of the following: abbreviations common to the clinical unit or clinical facility, safe administration of medications, and the appropriate documentation of care in the electronic medical record. For example, Dana drew upon their SCK in order to provide a clinical rationale to a student who was concerned because she could not, after several attempts, contact the patient's nurse via the telephone in order to report an abnormal assessment finding. Upon hearing the students' concern, 
Dana looked down at their watch, noted the time, and replied to the student "she [the nurse] didn't answer [their phone] because it is 8:30." The rationale behind this answer, as Dana explained to the student, was that the nurse that the student was attempting to contact was off the unit at a meeting held every day at 8:30 am for nurses who are in charge of the clinical unit. Through Dana's understanding of the every-day operations of the clinical unit, they were able to provide this student with clinical rationale and explanation for the nurse not answering the phone.

Responding: Providing Directions for Care. The participants were observed drawing upon their SCK numerous times in order to provide the students with directions for their nursing care including the safe administration of medications, correct and up-to-date documentation of nursing care, and the completion of nursing interventions. For example, Chris was reviewing the medication list in preparation for assisting a student to administer medications. Chris noticed a medication listed on the medication administration record that was a "high alert" medication. The clinical unit in which Chris was working had a specific policy that prohibited nursing students from administering this type of high alert medication due to the potential harm to the student. Based on their specialized knowledge of the specific unit policies and protocols, Chris provided the following directions to the student: "you need to tell the nurse [assigned to the patient] that she is going to have to give the medication. You are not able to give it."

Responding: Using Clinical Judgment. All of the participants utilized their SCK when using clinical judgment to facilitate student learning in the clinical setting. This knowledge was commonly used to assist students when administering medications along with keeping the patients safe and free from harm when implementing nursing interventions. As an example, when working in the clinical setting, a student approached Chris to ask about using a piece of 
assistive mobility equipment to assist the patient out of bed. To this, Chris responded "I would make sure, since this is his first time out of bed, that you ask the CA [clinical assistant] or the nurse [to help you]. In using their clinical judgment, as well as drawing upon their SPK, including knowledge of the roles, responsibilities, and scope of practice of other healthcare professionals, Chris provided directions to the student to secure assistance from the CA in order to safely move the patient out of bed.

Questioning: Asking about Completions of Tasks. All of the participants utilized their SCK when asking questions to inquire about the students' completion of nursing tasks or skills. These tasks or skills were dictated by a pre-set daily unit schedule and were based on the clinical units' policies or protocol. These questions were used to obtain information related to the students' timely completion of the following tasks: finger sticks, recording the patients' intake and output, documentation of daily assessment, and documenting hourly patient safety checks.

In summary, Specialized Content Knowledge involved the clinical nursing educators' knowledge of unit and hospital specific policies and procedures, as well as their understanding of the every-day operations of the clinical unit. This working knowledge facilitated the clinical nursing educators' ability to respond to and ask questions of the clinical students in order to provide safe and timely care. Additionally, this knowledge provided the clinical nursing educator with an understanding of the roles and responsibilities of other healthcare professionals, such as nursing assistants and other care providers.

\section{Horizon Content Knowledge}

Horizon Content knowledge (HCK) is an awareness of how topics are related over the span of time, how topics relate to previous courses, and how topics will relate to courses beyond. Horizon content knowledge is described as "an orientation to a familiarity within the discipline 
(or disciplines) that contribute to the teaching of the school subject at hand, providing teachers with a sense for how the content being taught is situated in and connected to the broader disciplinary territory" (Jakobsen, et al., 2012, p. 4642). A teachers' knowledge of the mathematical horizon may support them in making judgments about what is important to teach and orienting their instruction to the discipline (Jakobsen et al., 2012). When adapted to the Pedagogical and Subject Matter Knowledge for Clinical Nursing Educators framework, HCK was used to describe the clinical nursing educators' knowledge of what content is important to each in the clinical patient-care area. It also includes the clinical nursing educators' knowledge of how the content taught in the clinical patient-area connects to the students' prior knowledge and future learning. Examples of this would include the clinical nursing educators' ability to engage the students' prior knowledge from general education courses, such as the sciences and of preceding nursing courses. HCK would also include the clinical nursing educators' ability to relay information appropriate to the level of the learner.

Compared to the use of their Common and Specialized Content Knowledge, Horizon Content Knowledge was the least frequently observed type of knowledge utilized by the participants during the observational sessions. The participants were never observed alluding to or directly engaging the students' prior knowledge from their general education courses, including the pre-requisite science courses (i.e. chemistry, anatomy, or physiology), or prerequisite psychology or sociology courses. Although all of the participants were very familiar with the curricular content and course requirements of the nursing course in which they were currently teaching, they demonstrated very few examples to provide evidence that they were familiar with the content of nursing courses that had preceded their course and/or the content of courses that the students were enrolled in concurrently with their course. The exception to this 
was their understanding that the nursing students were either currently enrolled or had previously completed a course in Pharmacology.

It was not observed that the participants' judgment as to what to teach and when to teach it was based on their knowledge of how their teaching impacted the broader context of the curriculum or the students' understanding of the discipline of nursing. In fact, the participant's judgment regarding what was important or not important to teach was observed to be dictated by two distinct factors: the overarching academic level of the nursing student and the nursing care needs (including ordered medications, treatments, and interventions) of the patients for which the students were assigned. At times, the participants seemed unfamiliar with concepts that may or may not have been taught in the course prior to the one in which the participant was currently teaching. For example, when Chris was working with a student who was required to utilize a piece of medical equipment in the clinical setting, Chris asked the student "did you learn how to use a Doppler [the piece of medical equipment] in 211 [the nursing course that preceded this nursing course]?" For the novice clinical nursing educator participants, the lack of development or experience in using their Horizon Content Knowledge was observed influencing their failure, at times, to demonstrate to the students how the content taught in the clinical patient-area connects to the broader disciplinary territory. Likewise it effected the instructors' ability to connect the students past and present knowledge to their future learning. A further discussion concerning how this absence of knowledge influenced the teaching practices of the novice clinical nursing educator participants is provided in section three of this chapter.

\section{Summary of Subject Matter Knowledge}

In summary the participants in this investigation primarily drew upon their Content and Specialized Content Knowledge when responding to and questioning students in the pre-and 
post-conference settings and in the clinical setting. As described in this section, the participants drew least upon and demonstrated an unfamiliarly with the need to draw upon their Horizon Content Knowledge when teaching in the clinical setting. A summary of the context in which the participants utilized the facets of Subject Matter Knowledge is provided in Table 4.2 below.

Table 4.2

Context of the Use of Subject Matter Knowledge

\begin{tabular}{lccc}
\hline Subject Matter Knowledge & $\begin{array}{l}\text { Pre- } \\
\text { conference }\end{array}$ & $\begin{array}{l}\text { Post } \\
\text { Conference }\end{array}$ & $\begin{array}{l}\text { Clinical Patient } \\
\text { Care Area }\end{array}$ \\
\hline Common Content Knowledge & $\mathrm{x}$ & $\mathrm{x}$ & $\mathrm{x}$ \\
\hline Providing clinical rationale & $\mathrm{x}$ & $\mathrm{x}$ \\
\hline Using clinical judgment & $\mathrm{x}$ & $\mathrm{x}$ \\
\hline Providing direction for care & $\mathrm{x}$ & $\mathrm{x}$ \\
\hline $\begin{array}{l}\text { Asking for additional clinical } \\
\text { information or clarification. }\end{array}$ & & $\mathrm{x}$ \\
\hline $\begin{array}{l}\text { Asking about completions of } \\
\text { tasks }\end{array}$ & $\mathrm{x}$ & $\mathrm{x}$ \\
\hline Specialized Content Knowledge & $\mathrm{x}$ & $\mathrm{x}$ \\
\hline$\quad$ Providing clinical rationale & & $\mathrm{x}$ \\
\hline Using clinical judgment & $\mathrm{x}$ & $\mathrm{x}$ \\
\hline Providing direction for care & $\mathrm{x}$ & \\
\hline $\begin{array}{l}\text { Asking about completions of } \\
\text { tasks }\end{array}$ & & \\
\hline Horizon Content Knowledge & & \\
\hline Knowledge of Pharmacology & & \\
\hline
\end{tabular}

\section{Summary of Section Two}

This section of Chapter four provided an overview of how and when the participants drew from their Pedagogical Content Knowledge and Subject Matter Knowledge when teaching in the clinical setting. Real-world examples of teaching practices, including responses and questions derived from this knowledge, were provided. This section also revealed that the novice clinical educator participants were observed drawing heavily from their Common and Specialized Content Knowledge, however they demonstrated limited Horizon Content 
Knowledge when teaching nursing students in the clinical patient-care area. The final section of this chapter will address findings related to teaching practices that were either under-utilized or teaching practices that may have deterred student learning. A discussion of how the participants' lack of Horizon Content Knowledge along with other aspects of Pedagogical Content Knowledge, may influence their use of less than optimal teaching practices is provided in chapter five.

\section{Section Three: Teaching Practices that Deterred Student Learning}

As discussed in chapter two, according to the NLN Scope of Practice (2012) Competency

I, the responsibilities of an academic nurse educator in facilitating learning involves creating opportunities for learners to develop their critical thinking and clinical reasoning skills and implementing a variety of teaching strategies and practices appropriate to learner needs, outcomes, context and content. Additionally, the clinical nursing educator must have knowledge of and utilize teaching practices that facilitate nursing students' development of critical thinking skills in the clinical patient-care area setting (Dahlke et al., 2012). Numerous findings presented earlier in this chapter exemplify teaching practices that facilitated student learning in the clinical settings. However, asking questions that are above the level of the learner, the under-utilization of the teaching practice of questioning, and failure to engage the student in discussion were teaching practices used by the participants which may precluded the facilitation of student learning. The purpose of this final section of this chapter is to present a description of each of these less than optimal teaching practices, including examples.

\section{Asking Questions That Are Above the Level of the Learner}

During this investigation, the participants were observed asking numerous questions of their students. These questions were asked for the purposes of gaining additional clinical 
information or clarification, providing an understanding as to which tasks had or had not been completed, and facilitating students' recall or application of information. However, during the investigation, all of the participants were observed asking questions that were above the level of the of the student learner. These questions were unanswerable by the students, as they pertained to content or concepts to which they had not yet been introduced because of their academic level or their lack of clinical experience or exposure. The majority of the questions were asked during the pre-conference sessions. The following section includes examples of questions asked by each participant which students were unable to answer.

During a pre-conference session, Alex was observed working with a second-semester sophomore-level students in order to get a report on the students' assigned patients and posed the following question to the student group: "What are providers doing to curb patients from becoming addicted [to opioids]?" The question posed is from the perspective of a provider, and not from the perspective of a nursing student who has not yet been exposed to in-depth psychiatric or mental health content in their preparation to become a registered nurse. The sophomore-level students would not have insight as to what providers (i.e. nurse practitioners or medical providers) were doing to prevent patients from becoming addicted to opioids. As an additional example, while working in the pre-conference session with first-semester junior-level nursing students, Bailey was observed engaged a nursing student in a discussion concerning the use of an implanted central line port. During this exchange, Bailey posed the following question “As a new nurse, when you first graduate, can you access a port?" At this level, the student would not be familiar with skills they could or could not perform as a graduate nurse. Chris posed the following question to their clinical group: "Also, in surgery, do you think they leave these patients' dry [referring to their overall fluid status]?" The students were unable to answer 
this question, as they had not yet had an opportunity to observe the care of surgical patients in an operating room. Finally, Dana posed the following question to a group of first-semester junior students "Have you ever heard of graph vs host disease? If he was developing graph vs host, what kind of things would we see?" Unfortunately, this clinical content had not yet been introduced in the classroom setting to this level of student. Again, this level of questioning was above this level of learners and the students were unable to answer the question.

\section{Under-Utilization of Questioning as a Teaching Practice}

Clinical nursing educators are expected to facilitate the development of their students' critical thinking when teaching in the clinical patient-care area (Gaberson et al., 2015).

Questioning is a teaching strategy that may help develop nursing students' critical thinking skills (Gaberson et al., 2015; Holland \& Ulrich, 2016; Nicholl \& Tracey, 2007; Merisier, Larue, \& Boyer, 2018). As revealed in the second section of this chapter, the participants in this study were observed asking numerous questions of their students. However, on many occasions during the pre-conference sessions and in the clinical setting, the participants were observed providing information or responses to the student rather than posing a question that may be used to engage their prior knowledge and/or hold the students accountable for recalling and applying knowledge or concepts learned in previous courses in the clinical setting.

A review of the course syllabi for the courses in which the participants were teaching provided evidence that Pharmacology was a required nursing course to be taken during the second-semester sophomore semester. Therefore, the second-semester sophomore nursing students observed in this investigation were currently enrolled in Pharmacology, and the firstsemester junior level students would have successfully completed this course during the previous semester. Additionally, all students would have received didactic instruction pertaining to the 
process and principles of medication administration during their second-semester sophomore year. During the observational sessions, however, the participants were most frequently observed providing rationale, rather than using the teaching practice of questioning, in order to engage the students' prior knowledge and/or critical thinking skills related to principles of pharmacology and medication administration principles. By providing the students with information and rationale and not engaging in questions that would facilitate the students' recall or application of prior knowledge, the participants failed to provide opportunities for the students to develop their critical thinking and clinical reasoning skills related to pharmacology and/ or principles of medication administration (Gaberson et al., 2015).

Although all of the participants were observed under-utilizing or failing to utilize questioning to engage the students prior learning as a teaching practice, the following examples are provided from the clinical observations of Dana and Bailey. Dana and a first-semester junior nursing student were observed working in the clinical setting to prepare an inhaled medication via the use of a spacer. Concepts related to the use of an inhaler, including the use of a spacer, were introduced to the student in the second semester of the sophomore year. To begin the medication administration process, Dana asked the student if she could recall the clinical indication for the spacer. The student was unable to provide the information. Rather than holding the student accountable for their prior learning and/or asking additional questions to prompt the students' recall of this concept, Dana simply provided the student with the clinical rationale for the use of the spacer, along with clinical directions on how to correctly administer the inhaled medication. After administering medications with this student, Dana then moved on to the next student who was also required to administer medications. Dana asked the student to recall the pharmacological class of the medication. The student responded that the medication was in the 
ACE inhibitor class. Dana then asked the student what does "ACE mean and how does it work?" Like the first student, this student was unable to respond to this question. Rather than utilizing additional questions to engage the students' prior pharmacology knowledge, Dana simply provided the student with the clinical rationale for the mechanism of action and side effects of the medication. As an additional example, Bailey was observed working with a group of firstsemester junior students preparing medications to be administered via a nasogastric tube. Prior to medication administration, Bailey did not even attempt to engage the student's prior knowledge of this skill, rather they simply provided the student with the clinical information pertaining to the verification of placement of the tube, as well as the process of medication administration including the rationale for the use of liquid medications.

\section{Under-Utilization of Discussion or Reflection as a Teaching Practice}

As stated in chapter two, the primary role of the clinical nursing educator is to facilitate learning in the clinical patient-care area that bridges the gap between the classroom and the clinical setting (Dahlke et al., 2012). The teaching practice of discussion may be used to "promote the development of problem-solving, critical thinking, and clinical judgment skills"

(Gaberson et al., 2015, p. 260). As discussed in section two of this chapter, the participants were observed engaging their students in discussion and/or reflection, especially during the preconference sessions, in order to obtain clinical information about the patient. Additionally, the participants were observed asking the students to reflect on skills they had completed or observed during the clinical day. However, there were numerous occasions in which the participants were observed failing to recognize or act upon opportunities to engage their students in discussion or reflection in order to connect the students' didactic knowledge to the clinical setting and/or to facilitate critical thinking. Most commonly, the participants failed to facilitate 
discussion to make these connections during the pre-conference session, but this also occurred in the clinical setting, as well as in the post-conference setting. Although all of the participants were observed under-utilizing the teaching practice of discussion, the following examples are provided from the clinical observations of Alex, Bailey, and Chris.

Alex was observed working with a nursing student who asked for assistance in order to detect a heart murmur on their assigned patient. Alex responded to the students' request by entering the room. I observed Alex demonstrating to the student the correct placement of the stethoscope on the patients' chest wall. In doing this, the student was able to hear the abnormal sound that a heart murmur produces. However, Alex failed to engage the student in a discussion pertaining to the cause or implication of the patient's heart murmur on their overall health. As an additional example, during the pre-conference session, Bailey was observed getting a report from a student on a patient who was receiving Total Parenteral Nutrition or TPN. Bailey responded to the student by stating "what is TPN?" The student answered correctly by stating what the letters stand for, "Total Parenteral Nutrition". Upon hearing this, Bailey seemed satisfied with the students' response and directed the student to continue her report. Bailey did not engage the student or group in further discussion concerning the indications or side effects for this nutritional supplement. Finally, Chris was observed working with students in the pre-conference setting when a student reported that the patient he was to care for during the clinical day was presenting with a very low platelet count along with a history of liver cirrhosis. Rather than engaging the student in a discussion concerning the connections between or the clinical significance of these two abnormal findings or the implications these findings had to the care of the patient, Chris simply asked the student to continue on in their report. 


\section{Summary of Section Three}

In summary, the novice clinical nursing educator participants were observed utilizing many teaching practices when teaching in the clinical setting. However, they were not observed commonly or routinely utilizing teaching practices, such as questioning and discussion, which promoted or facilitated critical thinking and/or connected theoretical concepts learned in the classroom to the clinical setting, including promoting discussion and reflection. Additionally, the participants were observed routinely asking questions that were above the level of the learner.

\section{Similarities and Variances in Teaching Practices}

There were many observed similarities in the teaching practices utilized by the participants. For example, all participants demonstrated a wealth of clinical knowledge and expertise. As a whole, they appeared to be very comfortable and confident when teaching in the clinical setting. Each participant used a variety of teaching practices and adapted these teaching practices to the real-world context. As a group, the participants utilized low-level questioning as a teaching practice considerably more often than high-level questioning. Additionally, each participant asked questions that were above the level of the learner. Finally, as group, the participants tended to focus on completion of tasks in order to care for the patient during the clinical day, rather than engaging the students in higher-level critical thinking activities.

There were several observed differences in the teaching practices of the participant group. These differences were particular to the participants' methods of facilitating pre-and postconference sessions. For example, in the pre-conference setting, Chris was observed asking the students' numerous question concerning the students' pre-clinical work, including questions concerning the students' use of APA citations and formatting, as well as electronic sources they utilized to fully research the patients' health history. During the pre-conference sessions, the 
focus of Alex's questions were to primarily to probe the students' understanding of the nursing process, including asking about and providing constructive feedback concerning appropriate and inappropriate nursing diagnosis. Bailey was observed using a straight-forward question and answer approach to pre-conference that focused on receiving clinical information from the student, and providing clinical information when appropriate. During the preconference sessions conducted by Alex, Bailey, and Chris, the students were observed sitting quietly and only interacting with the clinical educator when called upon. Conversely, Dana was observed utilizing more informal, conversational communication techniques when teaching in the pre-conference setting, rather than a question-answer or probing approach. During the pre-conference sessions, Danas' clinical group was often observed engaging with each other and asking questions of their peers or of their instructor.

There were also observed differences in the methods the participants used to conduct post conference. Bailey was observed conducting very short, often times less than 15-minute, postconferences that involved asking the students to reflect briefly on the clinical day and then concluded with providing the students with directions for the following clinical day. Chris also asked the students to briefly reflect on their clinical day, but then provided their personal reflections and thoughts pertaining to the clinical day performances of individual students as well as the clinical group as a whole. Dana utilized original learning materials as well used pointed questions to facilitate student reflection of their clinical day. Finally, Alex used open-ended as well as pointed questions to facilitate student reflection of their day.

The variations amongst the participants in years of clinical and/or teaching experience was not observed to have a direct correlation to the participant's knowledge or utilization of teaching practices. For example, Dana, who had less than one year of teaching experience, was 
observed engaging the students in discussion or reflection more frequently than any of the other participants, each of whom had more teaching experience. Additionally, Dana, who had close to one year of teaching experience, and Alex, who had close to three years of teaching experience, developed and utilized original learning materials, where Bailey, who had close to one year of teaching experience, and Chris, who had close to three teaching experience, were not observed utilizing this teaching practice. It my proposition that that the participants' variation in teaching practices during the pre-conference sessions correlate more directly to their individual personalities and personal teaching preferences, rather than their underlying knowledge, grounding experiences, or years of teaching and/or clinical experience.

\section{Summary of Major Findings}

Multiple methods of data collection, including participant observation, ethnographic interviewing, and semi-structured interviewing, were used to answer the research questions. The findings demonstrated that the participants had a wide variety of clinical experiences prior to transitioning to the role of novice clinical educators. When teaching students in the clinical patient-care area, they drew from the knowledge and skills gained from their experiences as both a registered nurse and as a nurse practitioner or nurse practitioner student. The participants identified that their teaching practices were influenced by both positive and negative experiences with prior K-12 teachers, nursing professors, and trusted colleagues. Finally, it was observed that the participants differed in their perception of their role and responsibilities when teaching students in the clinical patient-care area.

The participants were observed utilizing multiple teaching practices, including two distinctive patterns of communication, responding and questioning, when working with undergraduate students in the clinical patient-care area. Although the participants were observed utilizing many successful teaching practices, they were also observed utilizing teaching practices 
that either failed to promote the development of critical thinking skills and/or failed to connect the theoretical concepts learned in the clinical setting. This included the under-utilization of discussion, reflection, and questioning, as well as asking questions above the level of the learner. When teaching in the clinical patient-care area, the participant group as a whole used similar teaching practices for which they commonly drew from their Common and Specialized Content Knowledge. In the clinical patient-care area, the teaching-learning process was primarily focused on the clinical needs of the patient, such as medication administration and nursing interventions. During the pre-and post- conference sessions, however, the teaching practices utilized by the participants in the varied greatly.

The Pedagogical and Subject Matter Knowledge for Clinical Nursing Educators framework, adapted from the Mathematical Knowledge for Teaching (Ball et al., 2008), was used to provide the guiding framework to identify and describe the participants' unique teacher knowledge. The participants were observed utilizing knowledge from five of the six facets of the Pedagogical and Subject Matter Knowledge for Clinical Nursing Educators framework: Knowledge of Content and Students, Knowledge of Content and Curriculum, Knowledge of Content and Teaching, Common Content Knowledge, and Specialized Content Knowledge. The participants were not observed routinely drawing on or demonstrating their Horizon Content Knowledge. The participants drew upon their Pedagogical Content Knowledge more often in the pre-and-post conference sessions, and less often when teaching in the clinical setting. Conversely, participants drew upon their Subject Matter Knowledge, displayed in the use of their Common and Specialized Content Knowledge, predominantly in the pre-conference sessions and when teaching in the clinical patient-care area. Further discussion and implication of these findings on nursing education practice and future areas of research follows in chapter five. 


\section{Conclusion}

The purpose of this chapter was to provide a rich description of the findings related to the knowledge, teaching practices, and grounding experiences of the four novice clinical educator participants. An introduction to each participant, including their unique nursing experience as well as personal and professional experiences that may have influenced those teaching practices was provided. An in-depth record of the teaching practices and context for those teaching practices was also offered. These teaching practices were correlated to the specific knowledge, based on the Pedagogical and Subject Matter Knowledge for Clinical Nursing Educators framework. Findings were shared that related to the teaching practices that were either underutilized or failed to promote the development of critical thinking skills and/or failed to connect the theoretical concepts learned in the clinical setting. Finally, a discussion of the similarities and difference of teaching practices utilized amongst the participant group as a whole was provided. 


\section{Chapter Five}

\section{Discussion and Conclusion}

\section{Introduction}

There were four research questions guiding this investigation. The purpose of this final chapter is to revisit these research questions and to provide a discussion of the major findings. Implications and recommendations for nursing education practice and for future nursing education are discussed. My final thoughts on the research process, findings, and implications conclude this chapter.

\section{Discussion of Major Findings}

Several major findings emerged from this investigation as they relate to the teaching practices, knowledge, and grounding experiences of novice clinical nursing educators. These findings include influences and perceptions of the role of the novice clinical educator, teaching practices that were under-utilized when teaching in the clinical setting, novice clinical nursing educator teacher knowledge, and the context in which specific facets of the novice clinical nursing educators' knowledge were demonstrated. A discussion of these major findings including the correlation of these findings to the original research questions follows.

\section{Influences on and Perceptions of the Role of the Novice Clinical Nursing Educator}

One of the four research questions guiding this investigation was: What grounding educational or professional experiences do novice clinical nursing educators refer to or draw upon when working in the role of the clinical nursing educator? As described in chapter four, each participant had unique grounding experiences, both personal and professional, that they draw upon when teaching in the clinical patient care area. These influences include their experiences as an undergraduate and graduate nursing student, experiences from their personal life, professional experiences as a registered nurse, as well experiences in working with more 
experienced colleagues when teaching in the clinical patient-care area. These findings are similar to other investigations of the teaching practices of novice clinical nursing educators. Scanlan (2001) and Dahlke, Baumbusch, Affleck, and Kwon (2012) reported that, without formal academic preparation in education, novice clinical nursing educators base their teaching on clinical and personal experiences, including previous experiences (both positive and negative) as a student, experiences with patient-centered educational sessions when working as a clinical nurse, and from working with other clinical teachers.

The perception of the role and/or responsibilities as a clinical nursing educator varied amongst the participants. According to the National League of Nursing's (NLN) (2012) Scope of Practice for Academic Nurse Educators, it is the expectation that clinical nursing educators facilitate the development of their students' critical thinking. However, when participants were asked about their perception of their role and responsibility of the clinical nursing educator, only one of the participants voiced that facilitating students' critical thinking was their primary role or responsibility when teaching in the clinical setting. Rather, the other participants' responses to their understanding of their role and/or responsibility as a clinical nursing educator varied from helping the students learn and complete tasks, to providing a connection from the didactic setting to the clinical setting, to creating a conducive learning environment. These variances represent an ambiguity in the participants' understanding of the role of clinical educator. Similar to the findings of this investigation, Owens (2018) reported that, when working in the role of educator, novice clinical educators may experience feelings of role ambiguity, feelings of confusion, and verbalize a lack of knowledge about teaching roles and responsibilities.

Benner (2001) suggests that the novice be given context-free guidelines or rules in order to regulate their practice. Unfortunately, the novice clinical nursing educator participants in this 
investigation began their teaching career with no previous experience, preparation, or graduate education in clinical teaching. Participants did not receive a formal orientation to include a definition of their role, and/or guidelines that may be used to govern practice as clinical educator. Without these guidelines or a clear understanding of their role and responsibilities, the participants assumed the role of a clinical expert and drew upon their previous personal and professional experiences, intuition, and background when teaching in the clinical patient-care area (Benner, 2001). These findings are similar to those of Andrew and Robb (2011) who found that clinical experts identified that were very comfortable in using their subject expertise when teaching in an academic setting but found it difficult to articulate their role and/or responsibly as an academic educator. Simply put, without a formal understanding of what or how they should teach, the participants' reverted to drawing upon knowledge that they would typically draw upon when functioning in the role of the clinical expert. This may provide insight as to why, when teaching in the clinical setting, the participants in this investigation drew much more from their common and specialized content knowledge, rather than the facets of pedagogical knowledge.

\section{Under-Utilization of Teaching Practices}

A second research question guiding this investigation was: What are areas of teacher knowledge and/or teaching practices utilized by novice clinical nursing educators, which preclude the facilitation of the development of student learning and/or critical thinking? The findings of this investigation demonstrated that knowledge and teaching practices were predominantly used by the participants in order to facilitate the implementation of safe and competent care to the patient and were not commonly used in order to engage students' higherlevel critical thinking. Discussion has been identified as a teaching practice that may be used in order to assist the student in identifying how or why difficult concepts are related to one another 
and/or facilitate critical thinking during the post-conference sessions (Oermann, 1997; Twibell, Ryan, \& Hermiz, 2005). However, facilitator-led discussion was a teaching practice that was not consistently or routinely used by the participants in the clinical and/or post-conference settings.

In the clinical setting, the novice clinical nursing educator participants were observed asking mostly recall or knowledge questions and under-utilizing asking in-the-moment higherlevel questions and/or initiating discussion that may be used to elicit critical thinking. Previous research also demonstrates that, in the clinical setting, clinical instructors ask more lower-level than higher-level questions that facilitate critical thinking (Hussey, Blackmore, \& McMurrary, 1998). The fact that the participants were novice educators may contribute to this finding, as Phillips and Duke (2001) found that educators with less teaching experience and no formal education in pedagogy are less likely to ask higher-level thinking questions when compared with the teaching practices of experienced nursing educators.

In the clinical patient-care setting, the participants' facilitated student learning that was, primarily, task-centered and dictated by nursing needs of the patients' provider orders, such as medication administration and other nursing interventions. Upon the conclusion of each clinical day, the participants would question students to ascertain which tasks had been performed during the clinical day and which tasks still needed to be completed. The findings of this study are consistent with the existing literature which revealed that interactions between the nursing student and the clinical nursing educator often focused more on tasks, such as assuring the student was doing their assigned work and assisting the student in medication administration (Hsu, 2006: Ironside, McNelis, and Ebright, 2014). For example, in the post-conference setting, instructor-led discussion often centered on the skills, tasks, or patient care performed during the 
clinical day. Similarly, Hsu (2008) reported that clinical instructors often focus their questions on pathophysiology and treatment.

The results of this and other investigations strengthen the argument that, when teaching in the "real-world" patient-care area, novice clinical nursing educators may prioritize skill or task completion in order to meet the clinical needs of the patients rather than implementing teaching practices, such as the use of questioning and reflection, that facilitate the development of higherlevel critical thinking skills. Ironside (2010) reported that clinical nursing educators often describe "teaching students to think on their feet and made clinical judgments" as the most significant challenge they face when teaching in the clinical patient-care area (p. 265). This phenomenon is not particular, however, to just novice clinical nursing educators, but may be particular to clinical experts who transition to the role of clinical educator. Kelly (2006) found that when teaching in the clinical setting, nursing educators with a clinically-oriented master's degree, such as the participants of this investigation, clinical nursing educators focused more on the in the nursing care of the patient rather than on teaching. Kelly (2006) attributed this difference to the clinically-focused faculty's expertise in the nursing care of the patient rather than on teaching.

\section{Teacher Knowledge and Context}

Two guiding research question centered on identifying and describing the teaching practices and underlying knowledge of those teaching practice used by clinical experts who transition to the role of novice clinical educator, as well as identifying how these teaching practices change in context. As the findings of this investigation demonstrated, the context of the learning environment was the strongest determinant of how and when the participants' utilized particular facets of subject matter knowledge, pedagogical content knowledge, and correlating 
teaching practices. For example, the participants were observed drawing on their pedagogical content knowledge, including knowledge of students, curriculum, and teaching, more often in the pre-and-post conference settings, and less often in the clinical settings. When teaching in the clinical setting the participants drew more consistently from the facets of subject matter knowledge, common and specialized content knowledge, when implementing teaching practices such as providing clinical rationale and providing directions for clinical care. These teaching practices were primarily utilized in order to facilitate the students' provision of safe and competent patient care. Additionally, the participants were dependent upon their ability to think critically in order to organize and oversee the students' implementation of patient care when working in the clinical setting.

The findings of this study indicated that the novice clinical educator participants were not familiar with and/or rarely drew upon the facet of horizon knowledge. Horizon knowledge includes the instructors' knowledge of how topics or concepts relate to previously learned concepts and/or how the content being taught is situated in and connected to the broader disciplinary territory (Jakobsen, et al., 2012). Keating and DeBoor (2018) suggest that all faculty members should be familiar with the total curriculum and student-learning outcomes for all levels of the nursing program. The participants of this study demonstrated curricular knowledge for the course they were presently teaching in that they had a working knowledge of the clinical assignments and appropriate skills that the student could or could not perform in the clinical patient-care area. However, outside of demonstrating their understanding that the students were either concurrently enrolled or had just completed a nursing pharmacology course, the participants did not consistently demonstrate the facet of horizon knowledge. During this investigation, the participants were observed asking questions that were above the level of the of 
the student learner. These questions were unanswerable by the students, as they pertained to content or concepts to which they had not yet been introduced because of their academic level or their lack of clinical experience or exposure. This may be, in part, due to the participant's lack of horizon knowledge, which includes a familiarity with concepts that were introduced in nursing courses that precede or that will be introduced in courses that succeed the course in which they are presently teaching.

Prior to this investigation, only a limited number of research studies were conducted to identify and describe clinical nursing educator knowledge. The results of these prior investigations, however, were not specific in identifying and describing the unique facets of teacher knowledge utilized by novice clinical nursing educators. Hsu (2008) utilized observational methods to examine the teaching practices of clinical nursing educators in Taiwan. However, the results pertained more to identifying facets of the educators' clinical knowledge rather than their knowledge of pedagogy. Other investigations examined different facets of teacher knowledge, including characteristic of effective teaching, and clinical nursing educators teaching strengths and weaknesses (Tang, Chou, \& Change, 2005: Wolf, Bender, Bietz, Weiland, \& Vito, 2004). However, the findings of these investigations were primarily founded on the nursing students or clinical faculty's perceptions of teacher knowledge, and not by observing inthe-moment clinical teaching. Wolf et al., (2009) developed the Clinical Teaching Knowledge Test, in order to assess clinical faculty's competencies and knowledge. Although the results of this test demonstrated that the participants scored, in general, poorly on the questions pertaining to education theory, this investigation did not shed light on other areas necessary for professional development of novice clinical educators. Unfortunately, the findings of these prior investigations were not particular to the specific knowledge of novice clinical nursing educators. 


\section{Limitations and Transferability}

In order to participate in this investigation, all participants were required to be novice clinical educators, with three years or less of teaching experience. Although all participants were considered novice educators, there were variances in their years of teaching experience, from less than one year to almost three years of teaching experience. Findings of this investigation indicate that that three years of less of teaching experience may not be adequate for novice clinical educators to identify their role and/or responsibility to facilitate critical thinking when teaching in the clinical patient area. Additionally, these variations amongst the participants' years of clinical and/or teaching experience was not observed to have a direct relationship to the participant's pedagogical knowledge or utilization of teaching practices that facilitate critical thinking. In fact, variation in teaching practices may be more directly related to the novice clinical educators' personal and professional experiences, and individual teaching preferences rather than their underlying knowledge, grounding experiences, or years of teaching and/or clinical experience.

These findings, un-anticipated by the researcher, may represent a limitation in the transferability in the findings of this investigation to the nursing education population at large. Additional limitations include the small number of participants (4) from a limited geographical area who were observed teaching. Additionally, all four participants were observed teaching sophomore or junior-level students in medical surgical courses, which may limit transferability to novice faculty teaching in other clinical areas. Finally, due to the observational presence of the researcher, who was known to all of the participants, the participants may have conducted their clinical teaching differently than if they were not being observed. 


\section{Conclusions and Recommendations for Future Practice}

A key factor in the success of nursing students is the instructional ability of the teacher

(Girija, 2012). Booth, Emerson, Hackney, and Souter (2016) argue that effective clinical nursing educators must have pedagogical preparation and utilize pedagogies that transcend the "what" questions, in order to explore the "why" questions. In other words, clinical nursing educators need to be knowledgeable of and competent in utilizing appropriate pedagogies when teaching nursing students the how's of nursing care (i.e. the tasks and skills), but, even more importantly, the whys of nursing care (i.e. theory) in order to facilitate the development of critical thinking skills. The findings of this investigation provides strength to the argument that, without formal preparation that includes information related to effective pedagogies, the novice clinical nursing educator may continue to draw upon past experience, personal teaching preferences, and trial and error well beyond their novice years. Therefore, clinical experts who transition to the role of novice clinical instructors must gain this information through formal mentoring and professional development programs to aid in there learning "on the job".

Jetha, Boschma, and Clauson (2016) suggest that identifying and addressing the needs of novice clinical nursing educators is a starting point to aid in the transition from clinical practice to novice educator. Historically, mentoring and new faculty development programs have primarily been developed and implemented to introduce and integrate new faculty to the role of the academic nursing educator (Shanta, Kalaneck, Moulton, \& Lang, 2011). When designing an orientation program for early career faculty members, the National League of Nursing (NLN) Board of Governors Position Statement on Mentoring of Nurse Faculty (2006) recommends including the following: an orientation to key personnel, a review of the course curricula, and courses, an overview of the job benefits, an introduction to the political environment and culture 
of the institution, and assigning a seasoned faculty member to serve as a formal mentor.

Additionally, professional development programs need to include instructional content pertaining to the distinct roles and responsibilities of the clinical nursing educator as well as how the role of clinical nursing educator differs from that of the clinical expert (Jetha, Boschma, \&Clauson, 2016). However, as the review of the literature has demonstrated, there is a gap in the existing literature related to the specific facets of knowledge that a clinical nursing educator needs to possess when teaching in the clinical setting. The findings of this investigation may help to fill that gap.

The findings of this study may be used as empirical evidence to begin to inform the field of nursing education of the underlying potential knowledge deficits of clinical nursing experts who transition to the role of a novice nursing educator teaching in the clinical patient-care area. The Pedagogical and Subject Matter Knowledge for Clinical Nursing Educators framework may be used as an integral part of the mentoring and professional development programs designed for novice clinical educators by identifying and describing the unique knowledge needed to be successful in the role of the clinical nursing educator. The framework may be used to identify the specific strengths and weakness of new clinical faculty pertaining to each unique facet of teacher knowledge. Additionally, the framework may be used as a guide in the creation of continued professional development programs that focuses on the identification and development of pedagogical knowledge and correlating teaching practices that may be used elicit critical thinking in the pre-and -post-conference settings, and even more importantly, in the clinical setting.

\section{Conclusions and Recommendations for Future Research}

The teaching practices used in the clinical patient-care area is one of the least studied areas in nursing education (Hossein et al., 2010; Phillips \& Vinten, 2010). As a review of the 
literature demonstrated, there have been limited investigations conducted to identify and describe the knowledge of clinical nursing educators. There is also limited research examining the knowledge of clinical experts, with no previous preparation in pedagogy or teaching practices, who transition to the role of the novice clinical nursing educator. Several authors have identified the need for continued research in the area of professional development of clinical educators (Hsu, 2008; Selleppah et al., 1998; Wolf et al, 2009) Ironside (2011) suggest that it is imperative that continued research be conducted in order to develop effective nursing education pedagogies and models of clinical education. Additionally, Phillips and Vinten (2010) suggest that further research is needed related to teaching strategies in order to better prepare future nurses to care for patients.

The results of this investigation provide several implications and recommendations for future research. This ethnographic investigation was the first of its kind to utilize the Pedagogical and Subject Matter Knowledge for Clinical Nursing Educators framework, adapted from the Mathematical Knowledge for Teaching framework (Ball et al., 2008), to define and describe novice clinical educator knowledge and correlating teaching practices. During this investigation, the framework was used to examine the teaching practices and knowledge of only four clinical nursing educators. Therefore, it is recommended that continued research be conducted to include larger number of participants in order to further refine the framework, as well as to pursue further research examining the teaching practices and knowledge of novice clinical nursing educators. Other recommendations include utilizing the framework to examine the teaching practices of clinical educators (both novice and experienced) teaching in specialty areas, such as pediatrics, obstetrics, and mental health. 
Another recommendation includes utilizing the Pedagogical and Subject Matter Knowledge for Clinical Nursing Educators framework to examine the knowledge and teaching practices of clinical nursing educators, novice through expert, who have completed graduate education in nursing education. There is a gap in the literature related to the teaching practices and underlying knowledge of clinical educators who have completed graduate education in nursing education. At this time, it is difficult to make comparisons between the teaching practices and knowledge of clinical nursing experts and those clinical nursing educators with a background in nursing education. Future investigations may utilize the framework to examine the knowledge and teaching practices of each of these educator groups in order to draw comparisons. These comparisons may be then used to further identify teaching practices and facets of knowledge that are unique to all novice clinical nursing educators, regardless of their graduate preparation. Additional research in this area may include researching the strengths and weaknesses of utilizing the Pedagogical and Subject Matter Knowledge for Clinical Nursing Educators framework in order to develop mentoring or faculty development programs. Finally, research may be done to identify and describe additional facets of knowledge utilized by clinical nursing educators.

\section{Imagining the Possibilities}

The findings of this singular investigation yielded results pertaining to the teaching practices and underlying knowledge of only four novice nursing educator participants. However, these findings may be utilized in order to plant the seeds for a change in culture related to the professional development practices used in the transition clinical experts to the role of novice clinical nursing educators. The following section describes the possibilities that may be explored through continued practice and research in these areas. 


\section{Development of Horizon Content Knowledge}

Identifying facets of knowledge that novice clinical nursing educators need continual development may be one of the first steps in developing continuing professional education. Horizon Content Knowledge (HCK) was a facet of knowledge, identified by this investigation, which the participants did not consistently demonstrate when teaching in the clinical patient-care area. As previously stated, Horizon Content Knowledge transcends course curricular knowledge, in that knowledge of how the content taught in the clinical patient-area connects to the students' prior knowledge and future learning. HCK include the clinical nursing educators' ability to engage the students' prior knowledge from general education courses, such as the sciences and of preceding nursing courses. HCK would also enable the clinical nursing educator to scaffold content in the clinical setting based on their knowledge of the broader disciplinary learning needs.

Horizon Content Knowledge may be developed by, first, by introducing novice educators to the distinct elements that comprise this facet of knowledge during the new-hire orientation program. This process may include introducing the nursing programs' curricular framework, including pre-requisite science courses. This introduction may include the sequence of nursing and non-nursing course, the course objectives of each course, and particular nursing skills and interventions that are evaluated in each course. Finally, information pertaining to the nursing programs overall student learning outcomes may be introduced during this orientation period. Follow-up in with the novice educator may be done in this area through working with a trusting and experienced mentor. This may also include the nursing educator shadowing the mentor during clinical days in order to gain a sense of what the nursing students should know or concepts and skills they may not yet be introduced. Finally, a formal follow up at the end of the 
semester may be suggested in order to facilitate the novice clinical nursing educators' selfreflection on their teaching and their overall development of Horizon Content Knowledge.

\section{Role of the Clinical Nursing Educator}

The results of this investigation demonstrated that novice clinical nursing educators may be conflicted in defining or understanding their role and responsibilities when teaching in the clinical patient care area. This may be due, in part, to their familiarity and ease in working in the role of the nurse in the clinical patient-care area and their unfamiliarity in working in the role of the clinical educator. Several steps may be taken to address this issue. First, during new hire orientation, the novice clinical nursing educators may be introduced to the National League of Nursing (NLN) (2012) Scope of Practice for Academic Nurse Educators. This framework may be used to introduce the novice nursing educator to the role and responsibilities of an academic nursing educator. Once introduced, purposeful discussion related to the similarities and differences in the role of clinical nurse and clinical educator may follow. Continued professional development may also include convening a panel of novice and expert clinical nursing educators in order to share their challenges, experiences, successful methods that they utilizes in order to transition from a clinical expert to a nursing educator. Finally, developing and utilizing "realworld" scenarios in order to conduct role-playing session may be used as part of the continuing professional process. These real-world scenario would allow the novice clinical educator the opportunity to confront situations where they would need to choose to perform in the role of clinical educator or clinical nurse. Feedback and discussion related to the educators' decision and actions during the role play would be provided during a debriefing session immediately following the role-play session. 


\section{Utilization of Simulation}

An additional suggestion for future professional development is to utilize the findings of this investigation and the Pedagogical and Subject Matter Knowledge for Clinical Nursing Educators in order to develop clinical simulations. Simulations are defined as "activities that mimic the reality of a clinical environment" (Jefferies, 2005, p. 87). Several investigations provide evidence that simulation may be used as a vital component in the professional development for novice clinical nursing educators (Hunt, Curtis \& Gore, 2015), Krautscheild, Kaakinen, \& Warne, 2008; Shellenbarger \& Edwards, 2012). The purpose or objectives of the simulations may vary, depending on the need of the clinical nursing educators. For example, to prepare the clinical expert for the role of the novice nursing educator, simulations may be written in order for the novice educator to experience the tension between acting in the role of expert nurse or in the role of clinical nursing educator. Simulations may also be developed to facilitate the novice clinical nursing educators' utilization of higher-level questioning, discussion, and reflection, when teaching in the clinical setting. Upon completion of the simulation, a structured debriefing session would be planned in order to discuss the novice clinical nursing educator's actions, teaching decisions, and behaviors during the simulation. Debriefing is the process in which participants of the simulation reexamine the encounter in order to foster the development of reasoning and judgment skills through reflective learning (Dreifurest, 2009). These simulations may be planned throughout the novice clinical nursing educators first year of employment and then on an as-needed basis depending on the novice educator's self-reflection of their practice as well based on their annual performance review.

None of the above recommendations have been proven to facilitate the transition of clinical nursing experts to novice clinical educators. They are merely educated thoughts on 
paper. However, these thoughts and ideas may benefit the nursing education community by beginning the conversation and imagining different ways to explore methods to facilitate the transition of clinical experts to novice clinical nursing educators.

\section{Final Thoughts}

There were several lessons learned and insights that were revealed to me during the process of conducting this study. Previous investigations that examined the teaching practices of clinical educators drew conclusions based on perceptions of nursing students or novice faculty themselves. However, as an experienced nursing educator, I was devoted to the idea of exploring alternate research methods in order to begin to understand what novice clinical educators really do and how they really teach in the clinical setting. In choosing to use ethnographic research methods, I committed to writing an accurate and factual record of these teaching practices. However, it was soon revealed to me that this method of research was both laborious and time consuming. However, using this research methodology in order to observe and record an up close and personal account of how and what novice clinical nursing educators are actually doing in the clinical setting was both revealing and exhilarating. By devoting hours observing novice clinical educators teaching in the clinical setting, a detailed and descriptive ethnographic record was constructed in order to begin to identify and describe the teaching practices and knowledge of novice clinical educators. I was unsure at first that this method was "going to work." However, by making the commitment to embrace this research method to its fullest, unique findings emerged from the research that, perhaps, may not have been revealed by using other research methodologies.

Secondly, several investigations have examined the lived experience of expert clinicians who transition to the role of novice clinical experts. Cangelosi, Crocker and Sorrell (2009) went so far as to coin the phrase, "expert to novice" to describe this transition. In order to succeed 
novice clinical nursing educators must have one foot in the realm of nursing and one foot in the realm of academia. However, clinical experts who transition to the role of novice clinical nursing educators are expected to competently perform in the role of nursing educator with no formal preparation or experience. The participants of this investigation were true clinical experts and did their best every day to provide their students with learning opportunities, guidance, and clinical rationale. However, they struggled to identify and utilize pedagogies that fostered and facilitated critical thinking. This lack of knowledge does not make them bad teachers, it just demonstrates areas of much needed mentoring and continuing professional development.

Over 100 years ago, Florence Nightingale, a historic figure who is considered to be the founder of modern nursing, created a model of nursing education based on her belief that it was imperative to teach future generations of nursing students not only what is to be done, but also why it should be done (Keating \& DeBoor, 2018; Nightingale, 1860). As the findings of this investigation revealed, even hundreds of years after Nightingale's decree, novice clinical nursing educators continue to struggle to find the balance between facilitating nursing students performance of the skills and interventions students need to provide patient care and facilitating the development of the critical thinking skills students need to perform these skills in the real world patient care setting. Unfortunately, over the past half-century, the focus of graduate nursing education has shifted from preparing academic nursing educators to preparing advanced practice nurses. This shift has led to the current generation of undergraduate clinical nursing educators, who are expert clinicians with a broad base of knowledge and skills needed to care for patients, but who may lack the pedagogical knowledge needed to inform teaching practices that facilitate critical and higher-level thinking in the clinical patient-care area. 
In conclusion, Nightingale was right. As a budding education researcher conducting this study, it was important to not only think about what research needs to be done, but also why it needed to be done. Shulman (2005) described the bedside teaching that occurs in the clinical patient-care area as the signature pedagogy of nursing education. This means that clinical teaching is the cornerstone of preparing future nurses for practice. The investment of time, energy, and devotion to conducting this research study yielded results that are, optimistically, both meaningful and impactful to nursing education. It is my hope that the findings of this investigation may add to the limited research pertaining to the signature pedagogies utilized by clinical experts who transition to the role of novice clinical nursing educator. Finally, it is my aspiration that the findings of this investigation, including the creation of the Pedagogical and Subject Matter Knowledge for Clinical Nursing Educators framework, play a small role in the advancement of professional development needed to facilitate the transition of clinical nursing experts to the role of the notice clinical nursing educator. 


\section{References}

Allan, H. T., \& Smith, P. (2010). Are pedagogies used in nursing education evident in practice? Nurse Education Today, 30, 476-479.

Altmann, T. K. (2007). An evalaution of the seminal work of Patricia Benner: Theory or philosophy? Contemp Nurse, 25(1), 114-123.

American Association of Colleges of Nursing [AACN] (2011). The Essentials of Master's Education in Nursing [Data file]. http://www.aacnnursing.org/Portals/42/Publications/MastersEssentials11.pdf.

American Association of Colleges of Nursing [AACN] (2016). Special Survey on Vacant Faculty Positions for Academic Year 2016-2017. [Data file]. http:// www.aacnnursing.org/Portals/42/News/Surveys-Data/vacancy16.pdf Shortage-Factsheet2017.pdf.

American Association of Colleges of Nursing [AACN] (2017a). Fact Sheet: Nursing Shortage. [Data file]. http://www.aacnnursing.org/Portals/42/News/Factsheets/Nursing- ShortageFactsheet-2017.pdf.

American Association of Colleges of Nursing [AACN] (2017b). Nursing Faculty Shortage Fact Sheet. [Data file]. http://www.aacnnursing.org/Portals/42/News/Factsheets/FacultyShortage-Factsheet-2017.pdf?ver=2017-07-11-103742-167

American Association of Colleges of Nursing [AACN] (2018a). Transitioning from Clinical Nursing to Nursing Faculty Tool Kit [Data file]. http://www.aacnnursing.org/TeachingResources/Tool-Kits/Transitioning-Clinical-Faculty.

American Association of Colleges of Nursing [AACN] (2018b). Master's Education. [Data file]. http://www.aacnnursing.org/Nursing-Education-Programs/Masters-Education. 
American Association of Colleges of Nursing [AACN] (n.d). Who we are [Data file]. www.aacnnursing.org/About-AACN.

American Association of Nurse Practitioners [AANP]. (2017). What's an NP? [Data file]. https://www.aanp.org/all-about-nps/what-is-an-np\#education-and-training.

Andreolli, K. (1987). Specialization and graduate curricula: Finding the fit. Nursing and Health Care, 8(2), 65-69.

Anderson, J. (2009). The work-role transition of expert clinician to novice academic educator. Journal of Nursing Education, 48(4), 203-208.

Andrew, N., \& Robb, Y. (2011). The duality of professional practice in nursing: Academics for the 21st century. Nurse Education Today, 31, 429-433.

Anibas, M., Brenner, G., \& Zorn, C. (2009). Experiences described by novice teaching academic staff in baccalaureate nursing education: A focus on mentoring. Journal of Professional Nursing, 25(4), 211-217.

Arkan, B., Ordin, Y., \& Yilmaz, D. (2018). Undergraduate nursing students' experience related to their clinical learning environment and factors affecting their clinical learning process. Nurse Education in Practice, 29, 127-132.

Backes, V., Moya, J., do Prado, M., Menegza, J., da Cunha, A., \& Fanscisco, B. (2013). Expressions of pedagogical content knowledge of an experienced nursing teacher. Texto \& Contexto Enfermagem, 22(3), 804-810.

Ball, D. L., Bass, H., Delaney, S., Hill, H., Phelps, G., Lewis, J., \& Zopf, D. (2005). Conceptualizing mathematical knowledge for teaching. In Annual Meeting of the American Educational Research Association, Montreal, Canada. 
Ball, D. L., Hill, H., \& Bass, H. (2005). Knowing mathematics for teaching: Who knows mathematics well enough to teach third grade, and how can we decide? American Educator, 29, 14-22.

Ball, D., Thames, M., \& Phelps, G. (2008). Content knowledge for teaching: What makes it special? Journal of Teacher Education, 59(8), 389-407.

Bandura, A. (1977) Social learning theory. Englewood Cliffs, N.J: Prentice-Hall.

Bastable, S. (2014). Nurse as Educator: Principles of Teaching and Learning for Nursing Practice. (4th ed.). Jones and Bartlett.

Benner, P. (2001). From Novice to Expert: Excellence and Power in Clinical Nursing Practice. Prentice Hall Health.

Benner, P. (2004). Using the Dreyfus Model of skill acquisition to describe and interpret skill acquisition and clinical judgement in nursing practice. Bulletin of Science and Technology, 24(3), 188-199.

Bethards, M. (2014). Applying social learning theory to the observer role in simulation. . Clinical Simulation in Nursing, 10(2), 65-69.

Beitz, J., \& Wieland, D. (2005). Analyzing the teaching effectiveness of clinical nursing faculty of full and part-time generic BSN, LPN-BSN, and RN-BSN nursing students. Journal of Professional Nursing, 21(1), 32-45

Billings, D., \& Halstead, J. (2009). Teaching in Nursing: A Guide for Faculty. Elsevier.

Billings, D., \& Kowalski, K. (2008). Developing your career as a nurse educator: The importance of having (or being) a mentor. The Jounral of Continuing Education in Nursing, 39(11), 490-491. 
Booth, T., Emerson, C., Hackney, M., \& Souter, S. (2016). Preparation of academic nurse educators. Nurse Education in Practice, 19, 54-57. doi:10.1016/j.nepr.2016.04.006.

Bowen, G. A. (2009). Document analysis as a qualitative research method. Qualitative Research Journal, 9(2), 27-40.

Braun, V., \& Clarke, V. (2006). Using thematic analysis in psychology. Qualitative Research in Psychology, 3, 77-101.

Brown, J., Collins, A., \& Duguid, S. (1989). Situated cognition and the culture of learning. Educational Researcher, 18(1), 32-42.

Brown, T., \& Sorrell J. (2017). Challenges of a novice nurse educator's transition from practice to classroom. Teaching and Learning in Nursing, 12(3), 207-211. doi:http://dx.doi.org.www.libproxy.wvu.edu/10.1016/j.teln.2017.03.002.

Cangelosi, P., Crocker, S., \& Sorrell, J. (2009). Expert to novice: Clinicians learning new roles as clinical educators. Nursing Education Research, 30(6), 367-371.

Cappelletti, A., Engle, J., \& Prentice, D. (2014). Systematic review of clinical judgment and reasoning in nursing. Journal of Nursing Education, 53(8), 453-458.

Carrega, J., \& Byrne, M. (2010). Problem-Based Scenarios to Learn Clinical Teaching Skills. Nurse Educator, 35(5), 208-212.

Clark, C. (2013). A mixed-method study on the socialization process in clinical nursing faculty. Nursing Education Perspectives, 14(2), 106-110.

Clark, N., Houten, L., \& Perea-Ryan, M. (2010). Transitioning from clinical practice to academia: University expectations for the tenure track. Nurse Educator, 35(3), 105-109. Commonwealth of Pennsylvania (2018). The Pennsylvania Code $\$ 21.369$ General Curriculum Requirements. https://www.pacode.com/secure/data/049/chapter21/s21.369.html. 
Conigliaro, R., \& Stratton, T. (2010). Assessing the quality of clinical teaching: a preliminary study. Medical Education, 44, 379-386.

Content Knowledge for Teaching. [Digital image]. Retrieved July 10, 2019, from ttps://www.google.com/search?tbm=isch\&source=hp\&biw=\&bih=\&ei=ck1XbGLBY6c5gLZu7uQCw\&q=content+knowlege+for+teaching\#imgrc=oUnXqH73o GKpdM.

Cooley, S., \& De Gagne, J. (2016). Transformative experience: Developing competence in novice nursing faculty. Journal of Nursing Education, 55(2), 96-100.

Creswell, J. (2018). Qualitative inquiry \& research design: Choosing among five approaches (Forth ed.). SAGE Publications.

Crookes, K., Crookes, P., \& Walsh, K. (2013). Meaningful and engaging teaching techniques for student nurses: A literature review. Nursing Education in Practice, 13(4), 239-243.

Crotty, M. (2015). The foundations of social research: Meaning and perspectives in the research process. SAGE.

Dahlke, S., Baumbusch, J., Affleck, F., \& Kwon, J. (2012). The clincial instructor role in nursing education: A structured literature review. Jounral of Nursing Education, 51(12), 692-696.

Dall'Alba, G., \& Sandberg, J. (2006).Unveiling professional development: A critical review of stage models. Review of Educational Research, 76(3), 383-412.

Denzin, N. K. (1989). Interpretive interactionism. Sage

Denzin, N., \& Lincoln, Y. (2018). The Sage handbook of qualitative research (5th ed.). SAGE.

DeWalt, K. M., \& DeWalt, B. R. (2010). Participant observation: a guide for fieldworkers. https://ebookcentral.proquest.com. 
Dornan, T., Boshuizen, H., King, N., \& Schierpbier, A. (2007). Experienced-based learning: A model linking the processes and outcomes of a medical students' workplace learning. . Medical Education, 41, 84-91.

Dreifurest, K. (2009). The essentials of debriefing in simulation learning: A concept analysis. Nursing Education Perspectives, 30 (2), 109-114.

Dreyfus, S. (2004). The five stage model of skill acquisition. Bulletin of Science and Technology. Dreyfus, H., \& Dreyfus, S. (1986). The power of human intuition and expertise in the era of the computer. The Free Press.

Dunham-Taylor, J., Lynn, C., Moore, P., McDaniel, S., \& Walker, J. (2007). What goes around comes around: Improving faculty retention through more effective mentoring. Journal of Professional Nursing, 25(6), 337-346.

Dupuis-Blanchard, S., Neufeld, A., \& Strang, V. R. (2009). The significance of social engagement in relocated older adults. Qualitative Health Research, 19(9), 1186-1195.

Emerson, R., Fretz, R., \& Shaw, L. (1995). Writing ethnographic fieldnotes. The University of Chicago Press.

Emerson, R., Fretz, R., \& Shaw, L. L. (2011). Writing ethnographic field notes (2nd ed.). University of Chicago Press.

Ensign, J., \& Bell, M. (2004). Illness experiences of homeless youth. Qualitative Health Research, 14(9), 1239-1254.

Facione, N., \& Facione, P. (1996). Externalizing the critical thinking in knowledge development and clinical judgement. Nursing Outlook, 44(3), 129-136.

Fenwick, T. (2000). Expanding conceptions of experiential learning: A review of the five contemporary perspectives on cognition. Adult Education Quarterly, 50(4), 243-272. 
Ferguson, L., \& Day, R. (2005). Evidence-based nursing education: Myth or reality? Journal of Nursing Education, 44(3), 107-115.

Fernandez-Balboa, J. \& Stiehl, J. (1995).The generic nature of pedagogical content knowledge among college professors. Teaching \& Education, 11(3), 293-306.

Fetterman, D. (1989). Ethnography step by step (Vol. 17). Newbury Park, CA: SAGE Publications.

Fraser, S. (2016). Pedagogical content knowledge (PCK): Exploring its usefulness for science lectures in higher education. Research in Science Education, 46(1), 141-161.

Gaberson, K., Oermann, M., \& Shellenbarger, T. (2015). Clinical Teaching Strategies in Nursing. (4th ed.). New York, NY: Springer.

Gardner, G., \& Harrelson, G. (2002). Situational needs: Meeting the needs of evolving learners. . Athletic Therapy Today, 7(5), 18-22.

Genrich, R., \& Pappas, A. (1997). Retooling faculty orientation. Journal of Professional Nursing, 13(2), 84-89.

Gess-Newsome, J., \& Lederman, G. (2001). Examining Pedagogical Content Knowledge. Kluwer Academic Publishers.

Gieselman, J., Stark, N., \& Farruggia, M. (2000). Implications of the situated learning model for teaching and learning nursing research. The Journal of Continuing Education in Nursing, 31(6), 263-268.

Giddings, L., Dyson, L., Entwistle, M., Macdiarmid, R., Marshall, D., \& Simpson, S. (2000). Three approaches to use questioning by clinical lecturers: A pilot study. Nursing Praxis in New Zealand, 15(1), 1322. 
Girija, K. M. (2012). Effective clinical instructor-A step toward excellence in clincial teaching. International Journal of Nursing Education, 4(1), 25-27.

Grossman, P. (1990). The making of a teacher: Teacher knowledge \& teacher education. Teachers College Press

Grossman, P., Schoenfeld, A., \& Lee, C. (2005). Teaching subject matter. In L. DarlingHammond \& J. Bransford (Eds.), Preparation of teachers for a changing world. JosseyBass.

Hanson, K., \& Stenvig, T. (2008). The good clinical nursing educator and the baccalaureate nursing clinical experience: Attributes and practice Journal of Nursing Education, 47(138-42).

Higginbottom, C. (2013). The use of focused ethnography in nursing research. Nurse Researcher, 20(4), 36-43.

Higginbottom, G., Pillay, J., \& Boadu, N. (2013). Guidance on performing focused ethnographies with an emphasis on healthcare research. The Qualitative Report, 18(9), 116.

Hill, H., Ball, D., \& Schilling, S. (2008). Unpacking pedagogical content knowledge: Conceptualizing and measuring teachers' topic-specific knowledge of students. Journal for Research in Mathematics Education, 39(4), 372-400.

Hinshaw, A. (2001). A continuing challenge: The shortage of educationally prepared nursing faculty. Online Journal of Issues in Nursing, 6(1), 3.

Hoffman, K., \& Donaldson, J. (2004). Contextual tensions of the clinical environment and their influence on teaching and learning. Medical Education, 38, 448-454 
Holland, C., \& Ulrich, D. (2016). Critical thinking cards: An innovative teaching strategy to bridge classroom knowledge with clinical decision making. Teaching and Learning in Nursing, 11(3), 108-112.

Horsfall, J. (1990). Clinical placement: Pre-briefing and debriefing as teaching strategies. The Australian Journal of Advanced Nursing, 8(1), 3-7.

Horsfall, J., Cleary, M., \& Hunt, G. (2012). Developing a pedagogy for nursing teachinglearning. Nurse Education Today, 32, 930-933.

Hossein, K., Fatemeh, D., Fatemeh, O., \& Katri, V. (2010). Teaching style in clinical nursing education: A qualitative study of Iranian nursing teachers' experience. Nurse Education Today, 10(1), 8-12.

Hsu, L. (2006). An analysis of clinical teacher behavior in a nursing practicum in Taiwan. Journal of Clinical Nursing, 15(5), 619-628.

Huber, S.W. (2018, April). Teaching strategies utilized by novice nursing educators. Poster session presented at the CEHS Celebration of Scholars, Morgantown, WV.

Hunt, C., Curtis, A., \& Gore, T. (2015). Using simulation to promote professional development of clinical instructors. Journal of Nursing Education, 54(8), 468-471.

Hutchings, P., \& Shulman, L. (1999). The scholarship of teaching: New elaborations, new developments. Change: The Magazine of Higher Learning, 31(5), 10-15.

Irby, D. (2014). Excellence in clinical teaching: Knowledge transformation and development required. Medical Education, 48, 776-784.

Ironside, P., \& McNelis, A. (2010). Clinical education in pre-licensure nursing programs: Findings from a national survey. Nursing Education Perspectives, 31(4), 264-265. 
Jakobsen, A., Thames, M., Ribeiro, C., \& Delaney, S. (2012). Using practice to define and distinguish horizon content knowledge. In 12th International Congress in Mathematics Education (12th ICME), 4635-4644.

Jefferies, P.R. (2005). A framework for designing, implementing, and evaluating simulations used as teaching strategies in nursing. Nursing Education Perspectives, 26(2), 96-103.

Jetha, F., Bochhma, G., \& Clauson, M. (2016). Professional development needs of novice nursing clinical teachers: A rapid evidence assessment. International Journal of Education Scholarship, 13(1), 1-10.

Judson, T., \& Leingang, M. (2016). The development of pedagogical content knowledge in firstyear graduate teaching assistants. Journal of STEM Education, 17(1), 37-43.

Kalensky, M., \& Hande, K. (2017). Transition from expert clinician to novice faculty: A blueprint for success. The Journal of Nurse Practitioners, 13(9), 433-439.

Karhuije, H. (1997). Classroom and clinical teaching in nursing: Delineating differences. Nursing Forum, 32(2), 5-12.

Kataoka-Yahiro, M., \& Saylor, C. (1984). A critical thinking model for nursing judgment. Journal of Nursing Education, 33(8), 351-356.

Kavilanz, P. (2018). Nursing schools are rejecting thousands of applicants in the middle of a nursing shortage. CNN Money. http://money.cnn.com/2018/04/30/news/economy/nursing-school-rejections/index.html.

Keating, S. B., \& DeBoor, S. S. (2018). Curriculum development and evaluation in nursing education (4th ed.). Springer Publisher, LLC

Kelly, R. (2006). Engaging baccalaureate clinical faculty. International Journal of Nursing Scholarship, 3(1), 1-16. 
Killam, L., \& Heerschap, C. (2013). Challenges to student learning in the clinical setting: A qualitative descriptive study. Nurse Education Today, 33(6), 684-691.

Kilian, C., Salmoni, A., Ward-Griffin, C., \& Kloseck, M. (2008). Perceiving falls within a family context: a focused ethnographic approach. Canadian Journal on Aging/La Revue canadienne du vieillissement, 27(4), 331-345.

Krautscheid, L., Kaakinen, J., \& Warner, J. R. (2008). Clinical faculty development: Using simulation to demonstrate and practice clinical teaching. Journal of Nursing Education, 49(9), 431-434.

Krefting, L. (1990). Rigor in qualitative research: The assessment of trustworthiness. The American Journal of Occupational Therapy, 45(3), 214-222.

Krisman-Scott, M. A., Kershbaumer, R., \& Thompson, J. (1998). Faculty preparation: A new soluation to an old problem. Journal of Nursing Education, 37(7), 318-320.

Kumi-Yeboah, A. \& James, W. (2012). Transformational teaching experience of a novice teacher. Adult Learning, 23(4), 170-177.

Loughran, J., Milroy, P., Berry, A., Gunstone, R., \& Mulhall, P. (2001). Documenting Science Teachers' Pedagogical Content Knowledge Through PaP-eRs. Research in Science Education, 31(2), 289-307. doi-org.www.libproxy.wvu.edu/10.1023/A:1013124409567

Lave, J. (1989). Cognition in Practice: Mind, mathematics and culture in everyday life. Cambridge University Press.

Lave, J. (1996). Teaching, as learning, in practice. Mind, Culture, and Activity, 3(3), 149-164.

Lave, J., \& Wegner, E. (1991). Situated Learning: Legitimate Peripheral Participation. Cambridge University Press. 
LeCompte, M. D., \& Schensul, J. J. (2010). Designing and conducting ethnographic research: An introduction (Vol. 1). Rowman Altamira.

Leininger, M. (1985). Qualitative research methods in nursing. Grune \& Stratton.

Lincoln, Y., Lynham, S., \& Guba, E. (2018). Paradigm controversies, contradictions, and emerging confluences, revisited. In N. Denzin, \& Y. Lincoln, (Eds.), The SAGE Handbook of Qualitative Research 5th Edition (108-149). SAGE Publications.

Lincoln, Y., \& Guba, E. (1986). But is it rigorous? Trustworthiness and authenticity in naturalistic evaluation. In D. D. Williams (Ed.), Naturalistic Evaluation. Josey-Bass.

Ludin, S., \& Fathulla, N. (2016). Undergraduate nursing students' perception of the effectiveness of teaching behaviors in Malaysia: A cross-sectional correlation survey. Nurse Education Today, 44, 79-85.

MacDougall, J., \& Drummond, M. (2005). The development of medical teachers: An enquiry into the learning histories of 10 experienced medical teachers. Medical Education, 39, 1213-1220.

Mann, K. (2011). Theoretical perspectives in medical education: Past experiences and future possibilities. Medical Education, 45, 60-68.

Mann, C., \& De Gange, J. (2017). Experiences of a novice clinical adjunct faculty: A qualitative study. The Journal of Continuing Education in Nursing, 48(4), 167-174.

McCurdy, D., Spradley, J., \& Shandy, D. (2004). The cultural experience: Ethnography in complex society. Waveland Press.

Meleis, A. (2010). Transitions theory: Middle-range and situational-specific theories in nursing research and practice. Springer. 
Melincavage, S. (2011). Student nurses' experiences of anxiety in the clinical setting. Nurse Education Today, 31(8), 785-789.

Merriam, S., \& Tisdell, E. (2016). Qualitative research: A guide to design and implementation (4th ed.). John Wiley \& Sons.

Mesirier, S., Larue, C., \& Boyer, L. (2018). How does questioning influence nursing students' clinical reasoning in problem-based learning? A scoping review. Nurse Education Today, 65, 108-115.

Miles, M., Huberman, M., \& Saldaña, J. (2014). Qualitative data analysis: A methods sourcebook. (3rd ed.). SAGE.

Miller, W. (2014). Developing a theory of clinical instructor identity using the experience of medical laboratory science practitioners. Clinical Laboratory Science, 27(2), 97-104.

Mishra, P., \& Koehler, M. J. (2006). Technological pedagogical content knowledge: A framework for teacher knowledge. Teachers College Record, 108(6), 1017-1054.

Mogan, J., \& Warbinek, E. (1994). Teaching behaviors of clinical instructors: An audit instrument. Journal of Advanced Nursing, 20(4), 160-166.

Morin, K., \& Ashton, K. (2004). Research on faculty orientation programs: Guidelines and directions for nurse educators. Journal of Professional Nursing, 20(4), 239-250.

Morse, J. (1994). Critical issues in qualitative research methods. SAGE Publications.

Morse, J., \& Richards, L. (2002). Readme first for a user's guide to qualitative methods. SAGE Publications.

Muecke, M. (1994). On the evaluation of ethnographies. In J. Morse (Ed.), Critical Issues in Qualitative Research Methods. Sage Publications

Munhall, P. (2007). Nursing research: A qualitative perspective. Jones and Bartlett 
National Advisory Council on Nurse Education and Practice [NACNEP] (2010). The impact of the nursing faculty shortage on nurse education and practice: Ninth Annual Report. [Data file]. http://www.hrsa.gov/advisorycommittees/bhpradvisory/nacnep/.

National League for Nursing Board of Governors (2002). Position statement: The preparation of nurse educators. [Data file]. http:// www.nln.org/docs/default-source/advocacy.../thepreparation-of-nurse-faculty.pdf.

National League for Nursing. (2005). The scope of practice for academic nurse educators.

National League for Nursing. (2012). The scope of practice for academic nurse educators. National League for Nursing Board of Governors (2017). Graduate Preparation for academic nurse educators [Data file]. http ww.nln.org/docs/defaultsource/about/nln-vision-series-(position-statements)/vision-graduatepreparation2.pdf?sfvrsn=8.

Nicholl, H., \& Tracey, C. (2007). Questioning: A tool in the nurse educator's kit. Nursing Education in Practice, 7(5), 285-292.

Nielsen, A. (2009). Concept-based learning activities using the Clinical Judgment Model as a foundation for clinical learning. Journal of Nursing Education, 48(6), 350-354.

Nightingale, F. (1860). Notes on nursing: What it is and what it is not. D. Appleton \& Co. http://www.digital.library.upenn.edu/women/nightingale/nursing/nursing.html

Nouri, J., Ebadi, A., Alhani, F., Rejeh, N., \& Ahmadizadeh, M. (2012). Qualitative study of humanization-based nursing education focused on role modeling by instructors. Nursing and Health Science, 15(2), 137-143.

Oermann, M. (1997). Evaluating critical thinking in clinical practice. Nurse Educator, 22(5), 2528. 
Oermann, M., \& Gaberson, K. (2017). Evaluation and testing in nursing education (5th ed). Springer.

Ortman, J. M., Velkoff, V., \& Hogan, H. (2014). The aging nation: The older population in the United States. [Data file]. https://www.census.gov/prod/2014pubs/p25-1140.pdf.

Owens, R. (2018). Two-year institution part-time nurse faculty experiences during role transition and identity development: A phenomenological study. Nursing Education Perspectives, $39(1), 10-15$.

Pasco, A. C. Y., Morse, J. M., \& Olson, J. K. (2004). Cross-cultural relationships between nurses and Filipino Canadian patients. Journal of Nursing Scholarship, 36(3), 239-246.

Patterson, B., \& Klein, J. (2012). Evidence for teaching: What are faculty using? Nursing Education Perspectives, 33(4), 240-245.

Patton, B. (2007). Knowing within: Practice wisdom of clinical nurse educators. Journal of Nursing Education, 46(11), 488-495.

Paul, P. (2015). Transition from novice adjunct to experienced associate degree nurse educator: A comparative qualitative approach. Teaching and Learning in Nursing, 10(1), 3-11.

Peters, S., Clarebout, G., van Nuland, M., Aertgeerts, B., Roex, A. (2018). A qualitative exploration of multiple perspectives on transfer of learning between classroom and clinical workplace. Teaching and Learning in Medicine, 30(1), 22-32.

Phillips, N., \& Duke, M. (2001). The questioning skills of clinical teachers and preceptors; A comparative study. Journal of Advanced Nursing, 33(4), 523-529.

Phillips, J., \& Vinten, S. (2010). Why clinical nurse educators adopt innovative teaching strategies: A pilot study. Nursing Education Perspectives, 31(4), 226-229. 
Poindexter, K. (2013). Novice nurse educator entry-level competency to teach: A national study. Journal of Nursing Education, 52(10), 559-566.

Pratt, D., Harris, P., \& Collins, J. (2009). The power of one: Looking beyond the teacher in clinical instruction. Medical Teacher, 31, 133-137.

Proto, M., \& Dzurec, J. (2009). Strategies for successful management and oversight of nurse faculty workforce initiatives: Lessons from the field. Journal of Professional Nursing, 25(2), 87-93.

Ramsburg, L., \& Childress, R. (2012). An initial investigation of the applicability of the Dreyfus Skill Acquisition Model to the professional development of nurse educators. Nursing Education Perspectives, 33(5), 312-316.

Roberts, K., Chrisman, S., \& Flowers, C. (2013). The perceived needs of nursing clinicians as they move into the adjunct faculty role. Journal of Professional Nursing, 29(5), 295-301.

Robinson, S. (2013). The relevancy of ethnography to nursing research. Nursing Science Quarterly, 26(1), 14-19.

Rohann, E., Taconis, R., \& Jochems, W. (2009). Measuring teacher's pedagogical content knowledge in technology education. Research in Science \& Technology Education, 27(3), 327-338. .

Roper, J., \& Shapira, J. (2000). Ethnography in nursing research (Methods in nursing research, v. 1). Sage Publications.

Saldaña, J. (2016). The coding manual for qualitative researchers (3rd ed.). SAGE.

Sanders, D., \& Welk, D. (2005). Strategies to scaffold student learning. Nurse Educator, 30(5), 203-206. 
Scanlan, J. (2001). Learning clinical teaching: Is it magic? Nursing and Health Care Perspectives, 22(5), 1-9.

Schaefer, K., \& Zygmont, D. (2003). Analyzing the teaching style of nursing faculty: Does it promote a student-centered or teacher-centered learning environment. Nursing Education Perspectives, 21(5), 238-245.

Schoening, A. (2013). From bedside to classroom: The nurse educator transition model. Nursing Education Research, 34(3), 167-172.

Schriner, C. (2007). The influence of culture on clinical nurses transitioning into the faculty role. Nursing Education Perspectives, 28(3), 145-149.

Sellappah, S., Hussey, T., Blackmore, A., \& McMurray, A. (1998). The use of questioning strategies by clinical teachers. Journal of Advanced Nursing, 28(1), 142-148.

Shanta, L., Kalanek, C., Moulton, P., \& Lang, T. (2011). Evidence for policy regulation: A model to address development of unqualified faculty. Policy, Politics, \& Nursing Practice, 12(4), 224-253.

Sharif, F., \& Masoumi, S. (2005). A qualitative study of nursing student experiences of clinical practice. BMC Nursing, 4(6), 1-7.

Shellenbarger, T., \& Edwards, T. (2012). Nurse educator simulation: Preparing faculty for clinical nurse educator roles. Clinical Simulation in Nursing Education, 8(6), e249-e255.

Shenton, A. K. (2004). Strategies for ensuring trustworthiness in qualitative research projects. Education for Information, 22, 63-75.

Shulman, L. (1986). Those who understand: Knowledge growth in teaching. Educational Researcher, 15(2), 14-14. 
Shulman, L. (1987). Knowledge and teaching: Foundations of the new reform. Harvard Educational Review, 57(1), 1-21.

Shulman, L. (2005). Signature pedagogies of the profession. Daedalus, 134(3), 52-52.

Smith, D., \& Neale, D. (1989). The construction of subject matter knowledge in primary science teaching. Teaching and Teacher Education, 5(1), 1-20.

Spiers, J. A., \& Wood, A. (2010). Building a therapeutic alliance in brief therapy: The experience of community mental health nurses. Archives of Psychiatric Nursing, 24(6), 373-386.

Spradley, J. (1970). You owe yourself a drunk: An ethnography of urban nomads (The little, brown series in anthropology). Little, Brown.

Spradley, J. (1972). Culture and cognition: Rules, maps, and plans (Chandler publications in anthropology and sociology). Chandler Pub.

Spradley, J. (1979). The ethnographic interview. Hardcourt College Publishers

Spradley, J. (1980). Participant observation. Hardcourt Brace Jovanovich.

Stevens, K., \& Duffy, E. (2017). A toolkit for nurisng clinical instructors. Teaching and Learning in Nursing, 12, 170-172.

Streubert, H., \& Carpenter, D. (2011). Qualitative research in nursing: Advancing the humanistic imperative (5th ed.). Wolters Kluwer Health/Lippincott Williams \& Wilkins.

Strike, K., Anderson, M., Curren, R., Van Geel, T., Pritchard, I., \& Robertson, E. (2002). Ethical Standards of the American Educational Research Association: Cases and Commentary.

Sweet, L., \& Broadbent, J. (2017). Nursing students' perceptions of the qualities of a clinical faciltator that enhanced learning. Nursing Education in Practice, 22, 30-36. 
Szymanski, M., \& Morrell, P. (2009). Situated cognition and technology. The International Journal of Learning, 15(12), 55-58.

Tang, F., Chou, S., \& Chiang, H. (2005). Students' perceptions of effective and ineffective clinical instructors. Journal of Nursing Education, 44, 187-193.

Tanner, C. A. (2006). Thinking like a nurse: A research-based model of clinical judgment in nursing. Journal of Nursing Education, 45(6), 204-211.

Thacker, E. (2017). PD is where the teachers are learning: High school social studies teachers' formal and informal professional learning. The Journal of Social Studies Research, 41, $37-52$.

Twibell, R., Ryan, M., \& Hermiz, M. (2005). Faculty perceptions of critical thinking in student clinical experiences. Journal of Nursing Education, 44(2), 71-78.

Valiee, S., Moridi, G., Khaledi, S., \& Garibi, F. (2016). Nursing students' perspectives on clinical instructors' effective teaching strategies: A descriptive study. Nurse Education in Practice, 16(1), 258-262.

van Driel, J. H., Verloop, N., \& de Vos, W. (1998). Developing science teachers' pedagogical content knowledge. Journal of Research in Science Teaching, 35(6), 673-695.

Van Maanen, J. (1995). Representation in ethnography. Sage Publications.

Van Maanen, J. (2011). Tales of the field: On writing ethnography (2nd ed., Chicago guides to writing, editing, and publishing). University of Chicago Press.

Weidman, N. (2013). The lived experience of the transition of the clinical nurse expert to the novice nurse educator. Teaching and Learning in Nursing, 8, 102-109.

West Virginia House Bill 4156, Article 7 Registered Professional Nurses § 30-7-5A Schools of Nursing Faculty Requirements. 
Wolf, Z., Bender, P., Beitz, J., Wiel, D., \& Vito, K. (2004). Strengths and weaknesses of nursing faculty as perceived by undergraduate and graduate students. Journal of Professional Nursing, 20, 118-128.

Wolf, Z., Beitz, J., Peters, M., \& Wieland, D. (2009). Teaching baccalaureate nursing students in clinical settings: Development and Testing of the Clinical Teaching Knowledge Test. Journal of Professional Nursing, 25(3), 130-144.

Young, P., \& Diekelmann, N. (2002). Learning to lecture: Exploring the skills, strategies, and practices of new teachers in nursing education. Journal of Nursing Education, 41(9), 405412.

Zungolo, E. (2004). Faculty preparation: Is clinical specialization a benefit or a deterrent to quality nursing education? Journal of Continuing Education in Nursing, 35(1), 19-23. 


\section{Appendixes}

Appendix A: Themes and Codes

Providing information/responding

- Giving directions for completion of assignment.

- Using visual and verbal instructions/ examples

- Providing direction for care.

- Providing clinical rationale.

- Utilizing their clinical judgment.

- Demonstration/hands on care

- Analogy

- Retrieving equipment

- Positive follow up from preconference.

\section{Questioning}

- Asking for additional clinical information/clarification.

- Non-clinical/ clinical assignment.

- Student reflection

- Questioning about completion of task( TASK) (BAD)

- Engagement (Knowledge/recall)

(Application)

(Critical thinking)

\section{Teaching via personal stories}

\section{Missed opportunity/ poor classroom}

- Missed learning opportunities.

- Failure to provide negative feedback.

- Poor classroom management.

- Unable to answer student questions.

- Failure to engage prior knowledge.

- Safe practice as underlying teaching practice.

- Self-perception as a novice

- TEACHING AS TASK (Made questioning @ tasks)

- Poor time management.

- Not upholding expectations

- No follow up in the clinical setting 
- Not holding students accountable for their own learning.

- Enabling

- Asking questions/providing information above the level of the learner.

- Greater emphasis on paperwork over patient care

- Poor pedagogy? (pitting students against each other, using students as poor examples in front of other students)

- Unfamiliar with clinical information

\section{Feedback}

Expectations.. .. could be via a form of a question ( green)

- Expectations too high for student

ROLE of the teacher

Engaging ( single student/ group)

\section{Classroom management}


Appendix B: Themes and Framework

\begin{tabular}{|c|c|c|c|}
\hline Q 1: & $2: 2$ & Q: 3 & Q: 4 \\
\hline $\begin{array}{l}\text { What teaching practices and } \\
\text { teacher knowledge } \\
\text { do novice clinical nursing } \\
\text { educators demonstrate or } \\
\text { draw from when teaching } \\
\text { undergraduate nursing } \\
\text { students in the clinical } \\
\text { patient-care area? }\end{array}$ & $\begin{array}{l}\text { In what ways } \\
\text { do novice clinical } \\
\text { nursing educators } \\
\text { change or adapt their } \\
\text { teaching behaviors as } \\
\text { a response to the } \\
\text { context of the } \\
\text { teaching/learning } \\
\text { environment? } \\
\text { Preconference: } \\
\text { Responding } \\
\text { Asking for additional } \\
\text { clinical } \\
\text { information/clarificat } \\
\text { ion. } \\
\text { Non-clinical/ clinical } \\
\text { assignment. } \\
\text { Giving directions for } \\
\text { completion of } \\
\text { assignment. } \\
\text { Providing clinical } \\
\text { rationale. } \\
\text { Utilizing their clinical } \\
\text { judgment. } \\
\text { Teaching through } \\
\text { personal stories } \\
\text { Providing feedback } \\
\text { Setting expectations } \\
\text { Questioning } \\
\text { Asking for additional } \\
\text { clinical } \\
\text { information/clarificat } \\
\text { ionNon-clinical/ } \\
\text { clinical assignment. }\end{array}$ & $\begin{array}{l}\text { What grounding } \\
\text { educational or } \\
\text { professional } \\
\text { experiences do novice } \\
\text { clinical nursing } \\
\text { educators refer to or } \\
\text { draw upon when } \\
\text { teaching } \\
\text { undergraduate } \\
\text { nursing students in } \\
\text { the clinical patient- } \\
\text { care area? }\end{array}$ & $\begin{array}{l}\text { Areas of continuing } \\
\text { professional development } \\
\text { for novice educators } \\
\text { Knowledge of } \\
\text { Content and } \\
\text { Curriculum. } \\
\text { Failure to engage prior } \\
\text { knowledge. } \\
\text { Knowledge of } \\
\text { Content and } \\
\text { Students. } \\
\text { Not holding students } \\
\text { accountable for their own } \\
\text { learning (telling rather than } \\
\text { asking). } \\
\text { Knowledge of } \\
\text { Content and } \\
\text { Teaching. } \\
\text { Missed Learning } \\
\text { opportunities } \\
\text { Failure to engage prior } \\
\text { knowledge. } \\
\text { Asking questions/providing } \\
\text { information above the level } \\
\text { of the learner. } \\
\text { Common Content } \\
\text { Knowledge }\end{array}$ \\
\hline
\end{tabular}




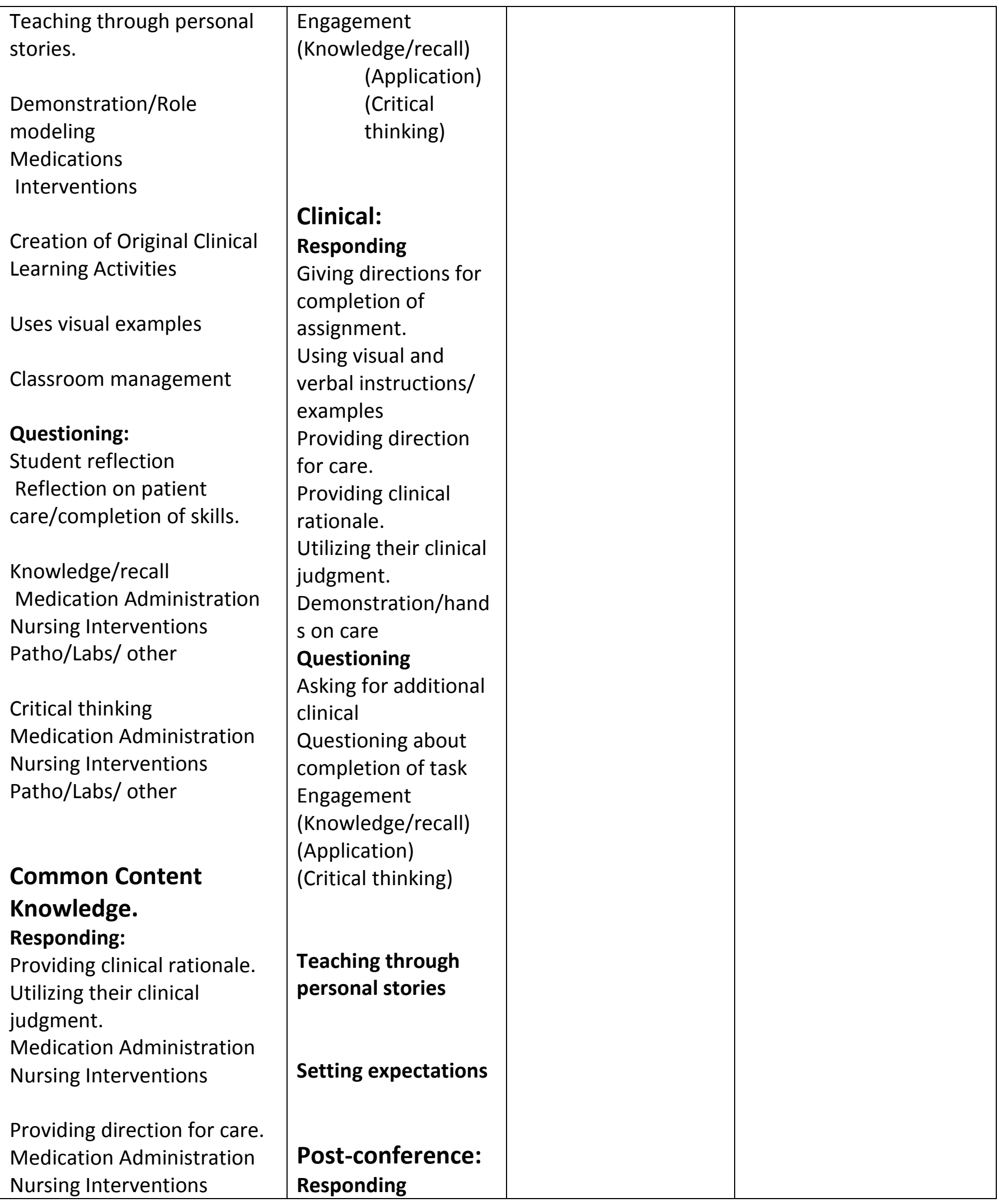




\section{Questioning:}

Asking for additional clinical information/clarification.

Medication Administration

Patient Information

Questioning about

completion of task.

\section{Specialized Content}

Knowledge

\section{Responding:}

Providing clinical rationale.

Medications

Interventions

Documentation

Providing direction for care.

Medications

Interventions

Documentation

Utilizing their clinical

judgment.

Interventions

Medications

Questioning:

Asking for additional clinical information/clarification.

Questioning about

completion of task

Horizontal Content Knowledge

$\begin{aligned} & \text { Giving directions for } \\ & \text { completion of } \\ & \text { assignment. }\end{aligned}$
Questioning
Asking for additional
clinical
information/clarificat
ion.

Giving directions for 


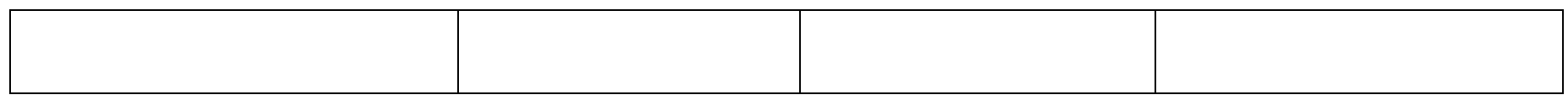


